\title{
How Far is Too Far? Understanding Identity and Overconformity in Collegiate Wrestlers
}

Ashley M. Coker-Cranney

Follow this and additional works at: https://researchrepository.wvu.edu/etd

\section{Recommended Citation}

Coker-Cranney, Ashley M., "How Far is Too Far? Understanding Identity and Overconformity in Collegiate Wrestlers" (2016). Graduate Theses, Dissertations, and Problem Reports. 5380.

https://researchrepository.wvu.edu/etd/5380

This Dissertation is protected by copyright and/or related rights. It has been brought to you by the The Research Repository @ WVU with permission from the rights-holder(s). You are free to use this Dissertation in any way that is permitted by the copyright and related rights legislation that applies to your use. For other uses you must obtain permission from the rights-holder(s) directly, unless additional rights are indicated by a Creative Commons license in the record and/ or on the work itself. This Dissertation has been accepted for inclusion in WVU Graduate Theses, Dissertations, and Problem Reports collection by an authorized administrator of The Research Repository @ WVU.

For more information, please contact researchrepository@mail.wvu.edu. 


\title{
How Far is Too Far? \\ Understanding Identity and Overconformity in Collegiate Wrestlers
}

\author{
Ashley M. Coker-Cranney \\ Dissertation submitted \\ to the College of Physical Activity and Sport Sciences \\ at West Virginia University \\ in partial fulfillment of the requirements for the degree of \\ Doctor of Philosophy in \\ Sport and Exercise Psychology
}

\author{
Jack C. Watson, II, Ph.D., Chair \\ Samuel Zizzi, Ph.D. \\ Dana Voelker, Ph.D. \\ Malayna Bernstein, Ph.D. \\ Jay Coakley, Ph.D.
}

Department of Sport and Exercise Psychology

Morgantown, West Virginia

2016

Keywords: deviance, overconformity, sport ethic, sport culture, athlete identity, wrestling, college, disorder

Copyright 2016 Ashley M. Coker-Cranney 


\title{
ABSTRACT \\ How Far is Too Far? Understanding Identity and Overconformity in Collegiate Wrestlers
}

\author{
Ashley M. Coker-Cranney
}

Although athletes who endanger the health and well-being of others are publicly shamed, those who endanger their own health and well-being in an effort to embody the sport ideal are often praised. Athletes are expected to distinguish themselves from their peers, make sacrifices for the good of the game, play through pain and injury, and push physical and mental limits on the path to achieve their goals (Hughes \& Coakley, 1991). Collectively, these expectations are known as the "sport ethic" and while they are considered part of sport culture, athletes who overconform to them may engage in behaviors that risk their health and well-being including disordered eating, chronic overtraining, and substance use. Although some research has investigated overconforming athletes' behaviors, overconformity to the sport ethic remains largely under researched (Coakley, 2015), despite an increasing prevalence of overconforming behaviors in the college athlete population. In an effort to examine athlete identity and deviant overconformity, the current study was designed within a psychocultural life story framework (Peacock \& Holland, 1993), using a constructivist-interpretivist paradigm (Ponterotto, 2005). Three collegiate wrestlers were interviewed using a life story interview protocol (adapted from McAdams \& Guo, 2014) and a semi-structured interview. Participants also completed the Athlete Identity Measurement Scale (Brewer \& Cornelius, 2001), the Social Motivation Orientation in Sport Scale (Allen, 2003), and a brief written expression exercise to provide context for participant narratives. Data were analyzed using provisional (Saldana, 2014) and narrative coding (Smith \& Sparkes, 2009b) and represented through the use of a word cloud (McNaught \& Lam, 2010) and creative nonfiction (Caulley, 2008; Sparkes \& Smith, 2014). Participants described a process of overconformity to the sport ethic that supported and extended previous research (Donnelly \& Young, 1988; Hughes \& Coakley, 1991). Results indicated that the wrestlers in the current study believed that, because athletes must push boundaries in order to find success, they cannot ever go "too far." Moreover, they reported that their athlete identity held significant personal and social meaning to the extent that they willingly engaged in behaviors associated with overconformity in previous literature (e.g., Atkinson, 2011; Johns, 1998; Waldron \& Krane, 2005). Recommendations for future research in this line of inquiry include similar studies with other sport populations (e.g., other sports, other competitive levels, other cultural backgrounds) and the development of a measure to identify athletes' degree of deviant overconformity. Practitioners may use this research to better conceptualize the healthcompromising behaviors their clients use to obtain athletic success, which may improve treatment planning and outcome goals. 


\section{Acknowledgements}

I would like to thank my family, friends, faculty, and mentors who helped me in this process, without whom I am certain I would not be where I am today. I thank Dr. Jack C. Watson, II for pushing me to be my best and reminding me that mistakes are part of the process. His tireless dedication to his students is extraordinary and I could not be more thankful for his professional guidance. I thank my committee members, Dr. Sam Zizzi who helped me keep an eye on the big picture and reminded me to take a critical lens to my work, Dr. Dana K. Voelker whose passion was contagious and kept me eager to share my work, Dr. Malayna Bernstein, without whom I would never have been able to finish such an ambitious methodological process - her encouragement and expertise were vital to the design and execution of this study, and Dr. Jay Coakley, whose life's work has been my inspiration for this project and whose support gave me strength. I would also like to Morgan Emter, whose research curiosity was contagious and research skills far surpassed that of an undergraduate and Dr. Edward Etzel, whose unwavering support and enthusiasm reminded me of the importance of my research. Thank you, too, to my colleagues and friends who read drafts, provided feedback, calmed my frustrations, and gave me pushes when I needed them most; thank you Dr. Chelsea Butters Wooding, Dr. Raymond Prior, Megan Byrd, Stefanee Van Horn, Meghan Halbrook, Tammy Sheehy, Leigh Bryant, Jay Stewart, Aaron Goodson, and Zenzi Huysmans.

As a final thank you, I thank my husband, son, and soon-to-be daughter for supporting me throughout this journey and reminding me of what is important in this world. They were a sanctuary when I was frustrated, a reminder of why I am passionate about my research, and a source of unconditional love and support that has pushed me follow my dreams. 


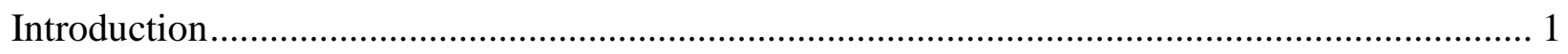

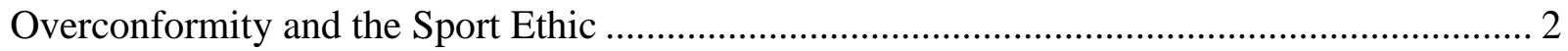

Culture of Overconformity to the Sport Ethic …………….............................................. 3

Individual Factors of Overconformity to the Sport Ethic.................................................... 5

Behaviors Associated with Overconformity to the Sport Ethic ................................................ 7

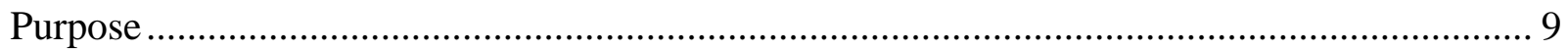

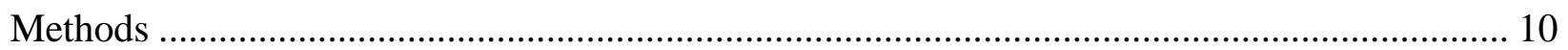

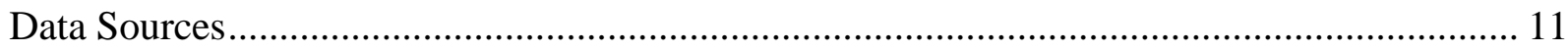

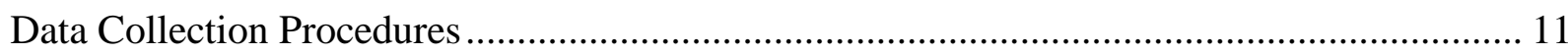

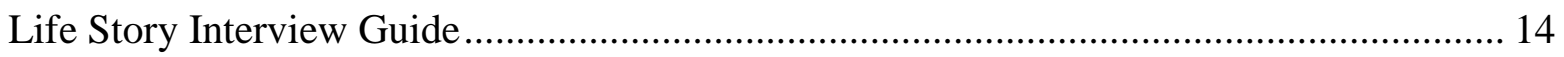

Athlete Identity Measurement Scale (AIMS) ………….............................................. 14

Social Motivation Orientation in Sport Scale (SMOSS) …………………………….......... 15

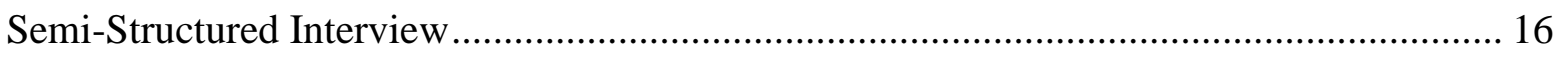

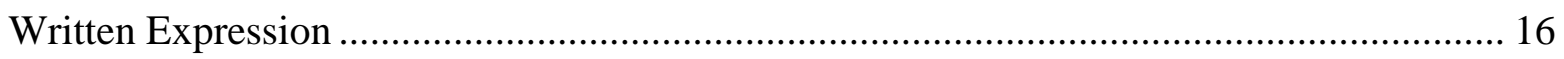

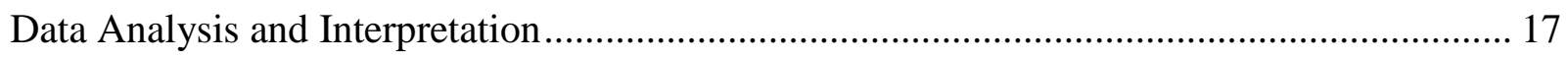

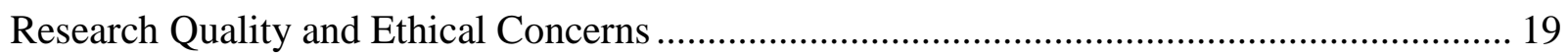

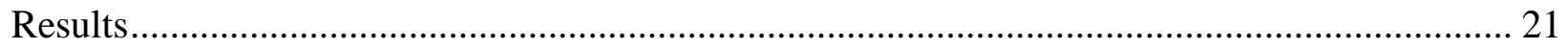

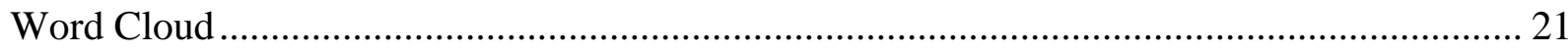

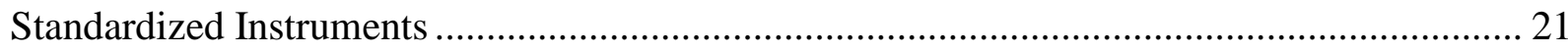

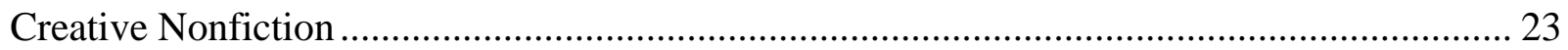

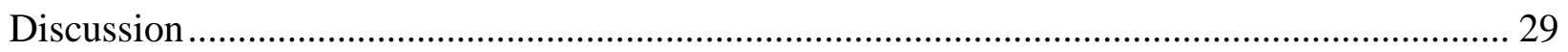

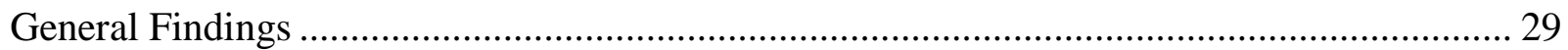

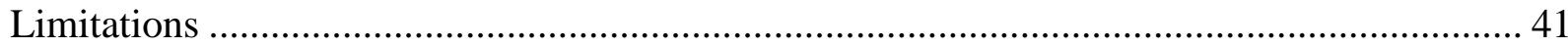

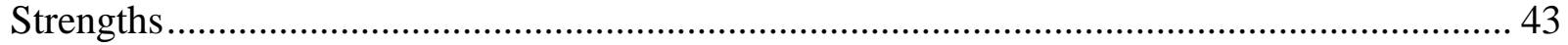

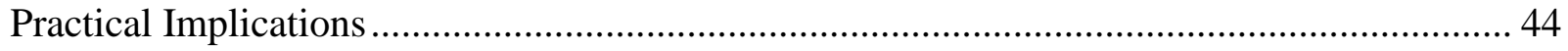

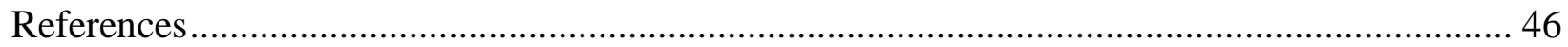

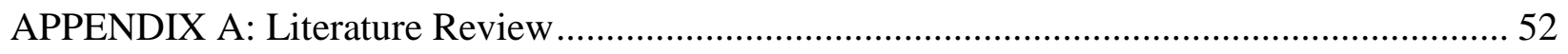

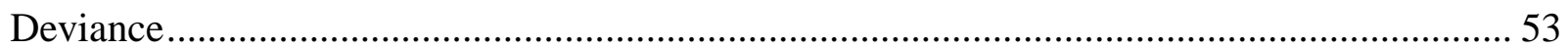




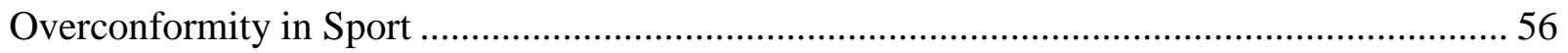

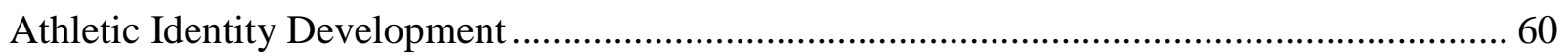

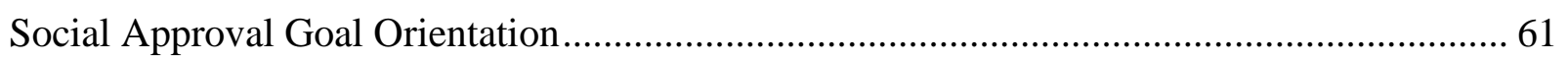

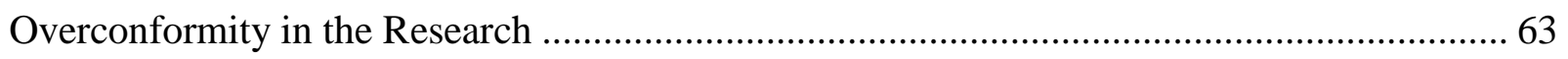

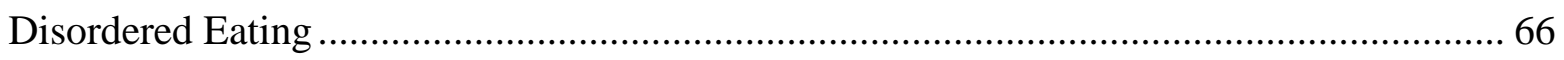

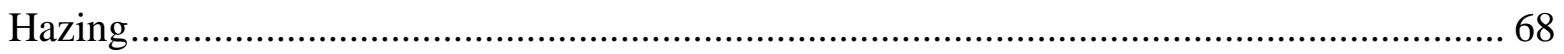

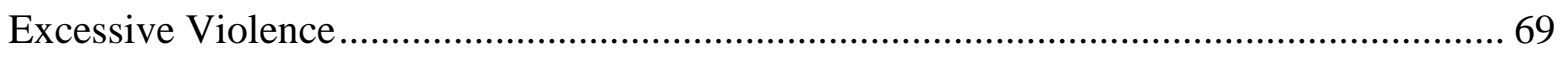

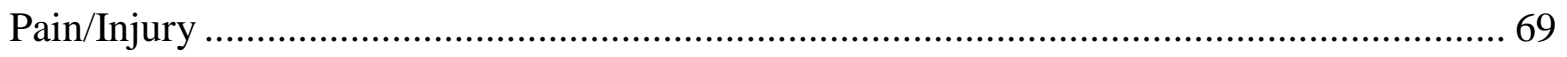

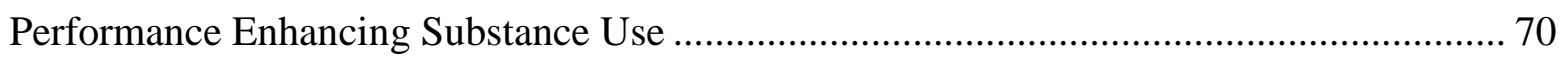

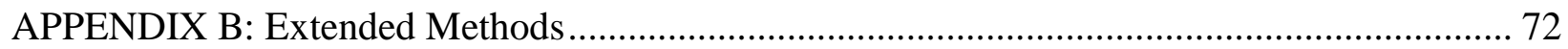

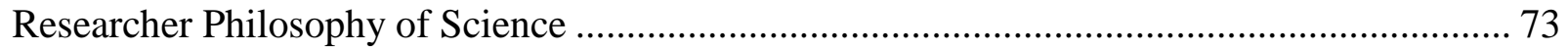

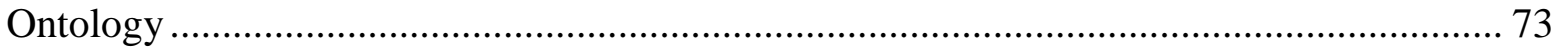

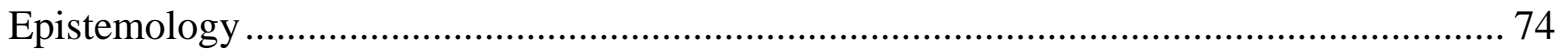

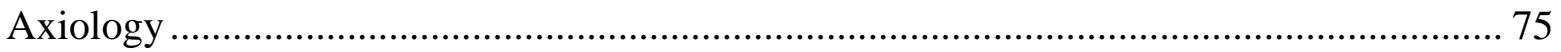

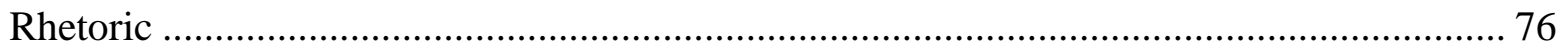

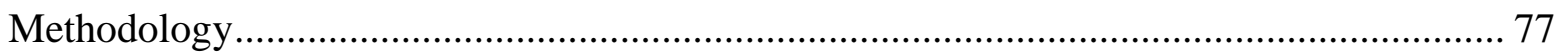

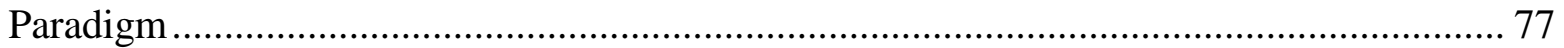

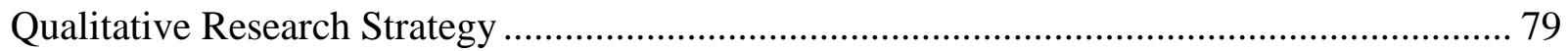

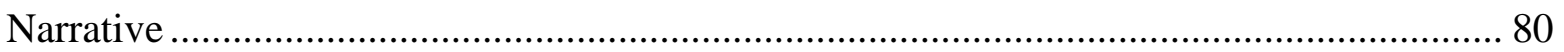

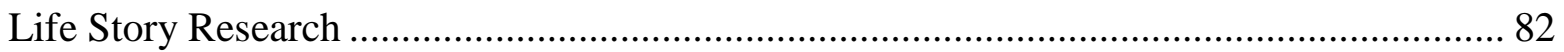

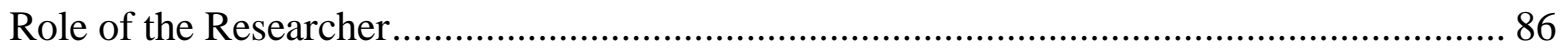

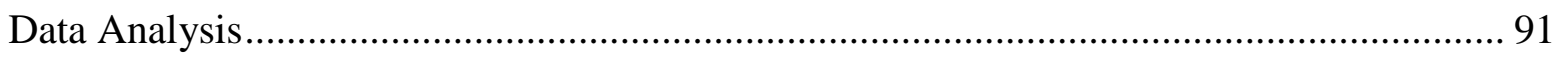

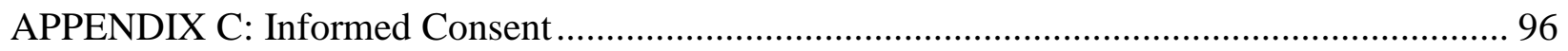

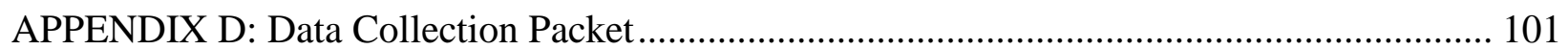

APPENDIX E: Provisional Coding Framework …………….................................................... 109

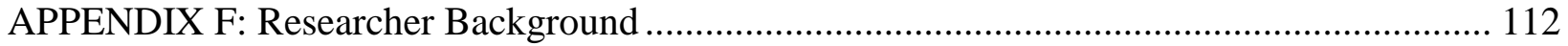

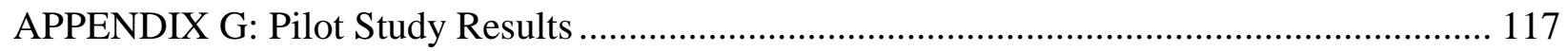

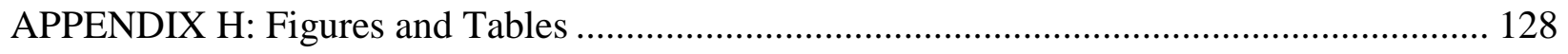

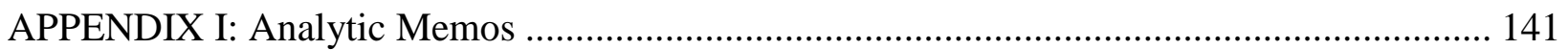




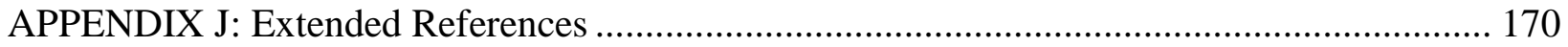




\section{List of Figures}

Figure

1 Conceptualization of Overconformity to the Sport Ethic in the Current

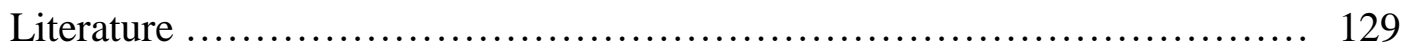

2 Prevalence Rates for Collegiate Athlete Behaviors Associated with Overconformity to the Sport Ethic .............................................. 130

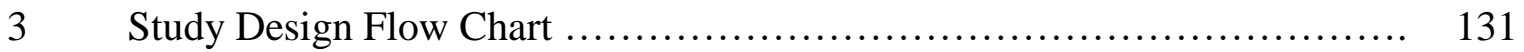

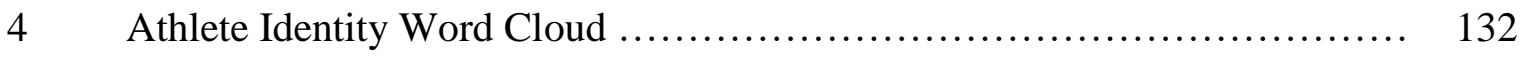

5 Overconforming Process for the Present Sample $\ldots \ldots \ldots \ldots \ldots \ldots \ldots \ldots \ldots \ldots \ldots \ldots \ldots$

6 Heckert and Heckert's (2002) Deviance Typology ...................... 134

$7 \quad$ Pilot Study Word Cloud ......................................... 135 


\section{List of Tables}

Table

1 Participant Recruitment Methods .................................. 136

2 Sample Participants' Demographic Variables for Maximum Variation ........ 140 
How Far is Too Far? Understanding Identity and Overconformity in Collegiate Wrestling Recently, Aaron Hernandez, former NFL tight end for the New England Patriots, was sentenced to life in prison without the possibility of parole following his conviction of first degree murder (Andersen \& Finucane, 2015). More distantly, UNC was investigated for a nearly 20 year long, institution-wide cheating scandal (Ganim \& Sayers, 2014). Ten years later, many are still talking about the infamous "Malice at the Palace" when Ron Artest, formerly of the Indiana Pacers, found himself in a brawl in the stands with Detroit Piston fans (Buckner, 2014). Although these behaviors are deviant from most athletes' and have been publicly shamed, other deviant behaviors are not only accepted, but glorified in sport.

Often, athletes who play while injured, deliver a particularly big hit, put in extra hours at the gym, lose more than prescribed weight in a short period of time, or put on a great deal of muscle mass in the off-season are praised by sport stakeholders (e.g., coaches, teammates, fans, family, media). While these practices are common in sport culture, at times, extreme consequences have forced governing bodies to change their policies regarding healthcompromising behaviors. For instance, disordered eating and exercise behaviors have historically been considered part of the wrestling subculture. Following the deaths of three otherwise healthy college wrestlers in 1997, the NCAA changed its rules regarding weight management in wrestling (Gibbs, Pickerman, \& Sekiya, 2009) to protect wrestlers from potential consequences of disordered eating and weight loss behaviors. Although it seems the rule change has been beneficial to many collegiate wrestlers (Opplinger et al., 2003), athletes - of all sports, at all levels - still engage in overconforming behaviors (e.g., disordered eating, disordered exercise, overtraining, chronic pain management, performance enhancing substance use) thought to improve performance but that can have devastating consequences for athlete health and well- 
being. Consequently, exploring overconformity by examining how the athlete identity is defined, communicated, ascribed meaning, and performed is necessary (see Figure 1 for a conceptualization of current literature related to overconformity to the sport ethic).

\section{Overconformity and the Sport Ethic}

According to Hughes and Coakley (1991), in order to gain and maintain athletic status, athletes must conform to the norms of the sport ethic, including: (a) striving for distinction, or seeking perfection, regardless of the costs, (b) sacrificing for "The Game," by prioritizing it above all other aspects in the athlete's life, (c) accepting risks and playing through pain and injury, to demonstrate their commitment and fearlessness, and (d) accepting no limits in the pursuit of excellence, regardless of the obstacles an athlete may face. The expectation of conformity may then increase athletes' vulnerability to engage in deviant acts (Coakley, 2015).Those athletes who overconform to the sport ethic uncritically accept its norms, engaging in certain behaviors in an effort to demonstrate their willingness to accept sport risks and prove their commitment to the athlete role. This deviant overconformity then affords the overconforming athlete a coveted status among sport stakeholders.

Behaviors of overconforming athletes, although theoretically rare, vary "in degree, not in kind" (Hughes \& Coakley, 1991, p. 316) from those of other athletes. For instance, whereas many athletes diet and exercise to improve performance, overconforming athletes may develop eating disorders (Johns, 1998). Whereas many athletes may take an ice bath or ibuprofen after training, overconforming athletes may undergo routine, high-dose injections of non-steroidal anti-inflammatory drugs (NSAIDs) or develop an addiction to prescription medication as a result of pain management efforts (King et al., 2014). Overconforming athletes even engage in hazing in an effort to fit in socially (Waldron, Lynn, \& Krane, 2011). 
Other studies have framed similar constructs within the performance narrative (e.g., Carless \& Douglas, 2013a, b). Similar to the distinction norm of the sport ethic, the performance narrative perpetuates the idea that positive performance outcomes distinguish the athlete as better than his competition. Stories of competitive triumph are then told and retold by other athletes, coaches, the media, and governing bodies, sending the message that the ultimate goal of sport is to set oneself apart as the best. These stories reinforce the idea that "winning is everything" (Carless \& Douglas, 2013a, p. 702) and that in order to be the best, sport must be the athlete's primary concern. This singular focus leads the athlete to funnel all available energy and resources, sacrificing other life roles and relationships, to attain the ultimate goal of being the best. Although overconformity is not discussed, per se, the sport ethic is demonstrated in athlete accounts of the performance narrative's effects on their lives.

Since the proposition of overconformity to the sport ethic, or deviant overconformity, in 1991, few studies have examined it explicitly (Coakley, 2015). Those that have found that despite athletes' knowledge of the risks associated with their deviant behaviors, the drive to continue to compete influenced them to engage (e.g., Atkinson, 2011; Fenton \& Pitter, 2010; Johns, 1998). At the core of many decisions to engage in overconforming behaviors is the socialized belief that commitment to the sport ethic provides the athlete mental (Pettersson, Ekstrom, \& Berge, 2012), physical (Atkinson, 2011; Fenton \& Pitter, 2010), and social advantages (Waldron \& Krane, 2005). Consequently, understanding the culture of sport is necessary.

Culture of Overconformity to the Sport Ethic. Sport has been described as a "culture of risk" (Nixon, 1992) whereby athletes are expected to voluntarily engage in certain risks for personal and social gains (Donnelly, 2004). Although sport risks may be economic or social in 
nature, the most prized sport risks are physical. The culture of risk, and expectation that athletes should assume risks in order to participate, is communicated to athletes via their "sportsnets" (Nixon, 2004, p. 84), consisting of social interactions between members of the sport community. Messages sent through sportsnets serve to explicate (a) expectations of the athlete role, (b) rewards for meeting those expectations, (c) sport values, (d) administrative acceptance of risk, (e) the athlete socialization process, and (f) explicit and implicit communication of the acceptance of physical risks (Nixon, 1993). Expectations of the culture of risk are communicated to athletes early and often by their sportsnets and are reinforced throughout their careers.

By conforming to the culture of risk, athletes perceive themselves to demonstrate the strength of their character, reaffirm their identity as athlete, and sustain delicate social bonds with teammates, coaches, fans, and other sport personnel (Donnelly, 2004). Overconforming athletes are placed upon pedestals, encouraging similar behaviors from aspiring athletes (Curry, 1993). Athletes unwilling to conform (e.g., the athlete who does not play injured in a big game) risk assaults to their character, undermining of their athletic identity, and isolation from significant others in sport.

The end product is that overconforming behaviors become a condition for membership within a sport subculture (Coakley, 2015). As a result of shared experiences with their teammates, and the conformity that was required to gain acceptance in the first place, athletes develop a deep sense of loyalty to one another. This loyalty can then lead to the sanctioning of additional overconforming behaviors, which strengthens the team's bond while also pressuring athletes to continue in their efforts to unquestioningly conform to the norms of the sport and the team (Atkinson, 2011; Johns, 1998; Waldron, Lynn, \& Krane, 2011).

In addition to peer acceptance, praise, and loyalty, institutional rationalization presents 
further pressure to overconform (Nixon, 1993). Coakley (2015) recently argued that the sport subculture makes athletes vulnerable to engaging in overconforming, and health-compromising, behaviors. As athletes progress to higher levels of competition and are expected to endure more intense training, they are afforded more extensive sport support staff who facilitate increased monitoring of athlete behaviors. This increased monitoring also presents increased pressure to conform within a closed subculture that prizes bodily sacrifice as a demonstration of commitment to the sport. By isolating athletes' social support to those within the sport subculture who support overconformity to sport norms, and ostracizing those professionals who disagree with it, overconformity is normalized and encouraged (Johns, 1998). Without outside support sources presenting alternative definitions of health and success, athletes may engage in healthcompromising behaviors tied to overconformity (Coakley, 2015).

Individual Factors of Overconformity to the Sport Ethic. For athletes who perceive their athletic identity to be important for maintaining their self-esteem, essential to gaining respect from others, or inextricably linked to their sense of masculinity (Coakley, 2015), overconforming is necessary. Consequently, "playing the game [is] much more than what athletes [do]—rather, it [is] who they [are]" (Coakley, 2015, p.388). Additionally, Waldron and Krane (2005) asserted that athletes are motivated to conform given a strong social approval goal orientation. Accordingly, actual identity may not be as important as peer social approval, which is rooted in hegemonic masculinity (i.e., dominant ideology of what it means to be a man that prompts athletes to feel the need to prove themselves; Donnelly, 2004; Nixon, 2004) and contingent upon strict adherence to team norms, including those norms of the sport ethic (Waldron, Lynn, \& Krane, 2011). Therefore, the identity of being an athlete may be an important driving factor for some overconforming athletes, but it may be a means to an end for other 
overconforming athletes with a strong desire to fit in with their peers.

If the athlete identity is of primary importance to the overconforming athlete, it is important to understand the socialization process. In sport, identity is proposed to develop through a process of identity construction and confirmation (Donnelly \& Young, 1988) which addresses the role of personal and social factors on identity development. Specifically, during the presocialization stage, the outsider is oriented to perceived behaviors and values of the desired group, which may not be consistent with actual group values. Then, the athlete is either selected (e.g., through the tryout process) or recruited to be a member of that group (i.e., selection/ recruitment stage). Once a member of the group, socialization begins whereby the athlete receives training about those behaviors and values expected by the group from established group members. Through the socialization stage, the athlete must choose whether to accept the values and behaviors prized by the sport subculture as a part of himself. Once the new member enters sport, his emerging identity is either confirmed or rejected by established members of the group on the basis of the new member's compliance with group expectations in the final stage of identity development (i.e., acceptance/ostracism stage). It is the last stage of identity development which seems to form a major motivation for deviant overconformity. As Hughes and Coakley (1991) note, athletes most likely to overconform are those with low self-esteem, and thus a strong need for acceptance from peers, those who perceive athletic success as an important way to gain the respect of others, and those who merge athletic identity and masculinity into a single identity. Therefore, the social confirmation of an athlete's identity, not winning, has been proposed to be the basis for normative overconformity (Coakley, 2015).

Other researchers (e.g., Waldron, Lynn, \& Krane, 2011) have suggested that the willingness to accept hegemonic masculinity and sport norms in an effort to gain social 
approval—not for the title of "athlete," but for the fulfillment of an individual's need for social approval-motivates the overconforming athlete. In the sport setting, specifically, athletes are driven to establish social bonds with their peers (i.e., social affiliation orientation) and receive validation of their identities from their peers by being successful in sport (i.e., social validation orientation; Allen, 2003). The needs for belonging, approval, acceptance, and status are most important for athletes with high social motivation orientation. If obtaining the status of "athlete" and engaging in overconforming behaviors is necessary to meet those needs, athletes are more likely to engage in behaviors such as hazing, disordered eating, excessive violence, and steroid use (Waldron \& Kowalski, 2009; Waldron \& Krane, 2005; Waldron, Lynn, \& Krane, 2011). Sport, then, becomes a site for overconformity, not the cause. Regardless of which motivation predominates, the process still requires strict adherence to team norms and the sport ethic, which may compromise athlete health and well-being.

\section{Behaviors Associated with Overconformity to the Sport Ethic. Understanding why} athletes overconform is of particular interest given the specific types of behaviors that have been proposed to be related to athletes' deviant overconformity. That is, Hughes and Coakley (1991) originally proposed that athletes who overconform are more likely to use performance enhancing substances and demonstrate excessively violent contact. Years after Hughes and Coakley (1991) proposed the concept of deviant overconformity, Waldron and Krane (2005) incorporated playing while injured and unhealthy weight management techniques, Coakley (2009) included overtraining that may cause bodily harm, and Waldron and Kowalski (2009) added hazing to the list of overconforming behaviors. Despite the theoretical rarity of deviant overconformity, such that it was suspected to occur at the far end of a normal curve (Coakley, 2009), prevalence rates for each (see Figure 2, Appendix H) exceed the predicted 2.5\%. For instance, in 2009, the 
NCAA found that approximately $5 \%$ of collegiate athletes used performance enhancing substances including amphetamines, anabolic steroids, and epinephrine (Bracken, 2012). Previous research has also indicated an increased intent by athletes who highly identify with the athlete role to use performance enhancing substances if it would mean the athlete would win a medal (Hale \& Waalkes, 1994). Vetter and Symonds (2010) found that one half of athletes surveyed experienced chronic injury during their last year of sport participation and one third reported frequent feelings of physical exhaustion, which was related to overuse syndrome, overreaching, overtraining, and resulting acute injuries. Additionally, 24.2\% of athletes surveyed in 2014 used prescription medication in the previous year, with 5.8\% doing so without a prescription (Rexroat, 2014). Moreover, Allan and Madden (2008) found that $74 \%$ of athletes surveyed reported having engaged in hazing in the last year. Combined, these rates indicate that deviant overconformity may be far more common than originally hypothesized.

Despite concern raised over the prevalence rates for behaviors thought to be the result of, or at least associated with, overconformity, it has not been well-studied. Since proposing deviant overconformity, few studies have explicitly used the framework to understand overconforming behaviors in athletics (Atkinson \& Young, 2008; Coakley, 2015). Those that have (e.g., Johns, 1998; Waldron \& Krane, 2005; Waldron \& Kowalski, 2009; Waldron, Lynn, \& Krane, 2011), have indicated a need to examine the process by which athletes overconform. Therefore, further research to understand deviant overconformity is necessary to guide future research and practice.

One issue that makes studying overconformity challenging is that many athletes see overconforming behaviors as normal (Coakley, 2009). That is, the line between athletes who appropriately conform to sport norms and those who go "too far" in their quest for athletic identity and/or social approval is often blurry. Thus, it is important to not only explore how 
deviant overconformity is developed, but also to determine at what point behaviors are considered deviant. Improved understanding about deviant overconformity may facilitate better education for athletes about those behaviors that may have a very real impact on their current and future health and well-being.

\section{Purpose}

The current study was designed to investigate wrestlers' experiences with the athlete identity and deviant overconformity. To address that aim, research questions focused on the definition, development, meaning, and performance of the athlete identity:

1. How do wrestlers define the athlete identity?

2. How are wrestlers socialized to understand the expectations of athlete identity?

3. What motivates wrestlers to overconform to the expectations of the athlete identity?

4. In what ways do wrestlers overconform in an effort to meet the expectations of the athlete identity?

Qualitative research has traditionally been conceptualized as inductive whereby a priori hypotheses are not appropriate. However, several researchers have explained that deductive qualitative research provides the researcher more opportunities to study complex phenomena and that the use of theory in qualitative research is particularly appropriate for research involving cultural phenomena (Creswell, 2009). Thus, the current study sought to use both inductive and deductive means for data collection and analysis to explore why some athletes engage in healthcompromising behaviors associated with deviant overconformity.

Several propositions were offered from a base of the current literature and the researcher's experience as athlete and coach to address the deductive nature of the study. First, the definition of "athlete", and expectations associated with the role of being an athlete, are 
communicated to incoming athletes through a socialization process similar to identity construction and confirmation as outlined by Donnelly and Young (1988). Second, the "athlete" identity is personally meaningful to the athlete because it represents a source of self-esteem, respect, and a sense of masculinity (Coakley, 2015) and secures peer acceptance (Waldron \& Krane, 2005). Third, because the "athlete" identity and resulting social approval is personally meaningful to the athlete, individual athletes are willing to demonstrate their commitment by striving for distinction, prioritizing athletic endeavors, accepting risks and playing through pain, and pushing limits (Hughes \& Coakley, 1991). Remaining information was sought inductively.

\section{Methods}

The purpose of the current study was to investigate the definition, development, meaning, and consequences of the athlete identity, as perceived by the participant, making a narrative design, using a psychocultural life story framework (Peacock \& Holland, 1993) within a constructionist-interpretivist paradigm (see Ponterotto, 2005) desirable. Consistent with the constructivist-interpretivist paradigm and narrative philosophy, the researcher and participants worked to co-construct the meaning of the participants' experiences of creating and performing the athlete identity (Corbin \& Strauss, 2008; Smith \& Sparkes, 2009a). Life story interviews, in particular, are desirable for the purposes of this study for a variety of reasons. Chief among those reasons, "[i]dentity itself takes the form of a story" (McAdams, 2001, p. 101). Moreover, life stories are selective whereby individuals, consciously or unconsciously, determine what events are highlighted in their life stories and what events are hidden (McAdams, 1998). Because they are selective, life stories inherently reflect individual values which are inextricably linked to cultural values and expectations (Peacock \& Holland, 1993). In these ways, the researcher was well-equipped to understand the identity development process, including socialization, which 
affected the meaning ascribed to the identity and its consequences for the individual. See Figure 3 (Appendix H) for a study design flow chart. A more detailed explanation of the methods is available in the Extended Methods (Appendix B).

\section{Data Sources}

For a narrative study using life stories, one to three participants is an appropriate sample size (Creswell, 2007; Carless \& Douglas, 2013b). Therefore, a purposive sample of three collegiate wrestlers comprised the participants for the current study. In accordance with guidelines for narrative research (Creswell, 2007), criterion sampling was used to select the participants. Inclusion criteria limited the current sample to collegiate varsity wrestlers at a Division I university, aged 18-23, with a desire to explore their athlete identities. These inclusion criteria were established for two reasons. First, the one criteria of narrative inquiry is that the participants are members of the culture of interest (Marshall \& Rossman, 2006); Division I varsity wrestlers have presumably spent considerable time in the sport subculture. Second, according to Erikson (1963), college-aged individuals are engaged in the identity versus role confusion challenge of human development (as cited in McAdams \& Guo, 2014) and experience a change in social relationships and societal expectations (Erikson, 1959, as cited in McAdams, 2001). Because life story is a means of identity formation, collecting data at the developmental point that individuals are integrating life experiences into a coherent story within a culturally meaningful context was ideal.

\section{Data Collection Procedures}

Following institutional review board approval, forty-two collegiate wrestlers were contacted via email up to three times and invited to participate in the study (see Appendix $\mathrm{H}$, Table 1). The goal of sending multiple emails was to recruit as many participants as possible to 
strive for maximum variation in the participant sample. In total, nineteen wrestlers responded to one or more of the email invitations, resulting in a $45.2 \%$ response rate. Of those who responded, nine wrestlers agreed to participate, six of whom completed data collection.

The six participants who provided all requested data spent $60-120$ minutes providing interview and written data for analysis; demographic information was also recorded for each (see Appendix H, Table 2). Three participants were chosen for analysis on the basis of maximum variation of the available demographic variables (i.e., those who contributed the greatest number of unique variables from the remaining sample). Consequently, the final sample represented three of four possible academic years, three of six weight classes, three of three geographic regions, two of two age groups when wrestling started, three of three wrestling team types, two of two state championship statuses, three of three sport combinations, two of three injury history types, two of three family member athlete statuses, and one of two parent-coach statuses (i.e., all three participants were coached at some point, in one or more sports, by a family member). Moreover, the final sample represented the widest range of scores on the Athlete Identity Measurement Scale (AIMS; Brewer \& Cornelius, 2001), Social Motivation Orientation in Sport Scale (SMOSS; Allen, 2003), and written expression. Data from the remaining three participants not chosen for analysis was stored on a password-protected computer for future analysis.

To collect data, participants met with the researcher on one or two occasions, depending on the availability of the participant. All data was collected in the same order: life story interview, AIMS, SMOSS, semi-structured interview, and written expression exercise. By using multiple methods, data was triangulated. As a result of collecting data in the specified order, it was funneled from general sport experience to specific sport experience related to the sport ethic and overconformity. Simultaneously, the specified order expanded from individual sport 
experience to the participants' observations of others athletes' sport experiences.

Specifically, the life story interview was intentionally general to prompt the athlete to share all sport-related experiences that may have contributed to the definition, development, meaning, and consequences of the athlete identity, accounting for the effects of non-sport stakeholders as well as different sport subcultures. The AIMS and SMOSS were then administered to assess each participant's level of identification with the athlete role and degree of social motivation orientation, respectively. The semi-structured interview was created to specifically address the research questions, without explicitly introducing the concept of overconformity to the participants and biasing responses. The written expression was then administered to specifically investigate overconforming behaviors. Both the semi-structured interview and written expression asked participants to speak to their individual experiences, as well as their observation of other athletes' behaviors. Doing so allowed the researcher to sense the possibility of the false consensus effect (FCE), whereby individuals are predisposed to overestimate the behaviors of others when they engage in those behaviors, themselves. Support for the FCE has been established in previous research that found that athletes who reportedly consumed more illicit drugs also reported that those around them consumed more illicit drugs (Dunn, Thomas, Swift, \& Burns, 2012). Given the sensitive nature of overconforming behaviors, it was important to shift the focus to others, if it made the participants more comfortable.

Although not an explicit purpose of the current study, the AIMS and the SMOSS were administered to measure levels of athlete identity and social motivation orientation, respectively. Doing so aided in developing an understanding of the meaning each participant associated with the athlete identity. Using the AIMS and the SMOSS to gauge each participant's level of identification with the athlete role and social motivation orientation provided the researcher with 
context for interpreting the narratives shared by participants, as well as important information when creating the character for the creative nonfiction who most appropriately represented each of the three participants in the study. Given the small sample size of the current study, achieving statistical significance was impossible. Instead, the goal was to use the AIMS and SMOSS as means of practical significance to better understand the meaning of participation in sport, related expectations, and behaviors disclosed by the research participants.

Data from all tape-recorded interviews were transcribed verbatim. Upon completion of transcription, audio files were erased to protect confidentiality. Transcribed interviews and written assessments were stored in a locked file drawer and on a password-protected computer, accessible only to the principle researcher and research team. Written informed consent was obtained at the first interview (see Appendix C). The researcher was not affiliated with any wrestling team.

Life Story Interview Guide. The first interview followed life story interview guidelines (adapted from McAdams \& Guo, 2014; see Appendix D) in order to understand the athletic experiences of each participant, as well as the meaning athletes ascribed to their experiences and identities. The life story interview aims to identify key moments in an individual's life, including past events, important people, future hopes, values, and overall life themes. This process creates a coherent story that reflects the participant's sense of self. Thus, as participants restructure past events and imagine future possibilities to form a coherent story, the researcher is provided a narrative which explains the participant's identity. Life story interviews have been conducted in a wide variety of disciplines, and recently, life story interviews have been used in sport psychology (e.g., Carless \& Douglas, 2013a, b) to investigate a range of participant experiences. Athlete Identity Measurement Scale (AIMS). To obtain context related to the level of 
importance participants in the current study placed on the athlete identity, each completed the short, 7-item version of the AIMS (Brewer \& Cornelius, 2001; see Appendix D). The AIMS was designed to assess the level of investment participants have with the athlete role, related to other possible life roles. Participants ranked each item on a 7-point Likert scale from 1 (strongly disagree) to 7 (strongly agree). The 7-item version of the AIMS has been validated with athletes and non-athletes (alpha $=.81 ; 2001)$. Brewer and Cornelius $(2001)$ also provided norms to which researchers and practitioners can compare the scores of their participants and clients. Although it is comprised of three subscales (i.e., Social Identity, Exclusivity, and Negative Affectivity), the entire AIMS score was used for the current study given the small sample size and purpose of data triangulation. Normative comparisons were made for each of the participants to aid the researcher's coding efforts and better understand the holistic context of each athlete's experiences. Normative comparisons were also examined for the sample, collectively, to create an appropriate level of investment for the character portrayed in the creative nonfiction.

Social Motivation Orientation in Sport Scale (SMOSS). To obtain context related to the degree to which participants in the current study were motivated to socialize and reap social gains, each completed the 15-item SMOSS (Allen, 2003; see Appendix D). Items on the SMOSS were ranked from 1 (strongly disagree) to 5 (strongly agree). Internal reliability for the SMOSS was established at alpha $>.70$ in previous research (i.e., Allen, 2003). Similar to practical analysis of the AIMS, SMOSS scores were analyzed independently, to aid in coding individual data, and collectively, to aid in the development of the creative nonfiction character. Further, SMOSS results were analyzed with respect to each subscale. Specifically, it was important to understand the degree to which athletes were motivated by (a) Affiliation, or the desire to build satisfying peer relationships, (b) Social Status, or the goal to obtain popular standing among 
peers, and (c) Social Recognition, the drive to be recognized for one's accomplishments. Given that existing research has not normed the SMOSS with male collegiate athletes, it was inappropriate to compare the results of this study with previous studies using the SMOSS. However, the information gathered provided the researcher with a practical level of understanding of the importance of sport to the participants, as related to social motivations of belonging, popularity, and recognition.

Semi-Structured Interview. A semi-structured interview was intended to complement the life story interview. In contrast to the open-ended nature of the life story interview, the semistructured interview (see Appendix D) created for this study addressed information related to specific research questions. Each interview question was structured to ask the participant to first speak about his observation of other athletes, and then himself, in an effort to reduce the pressure he might feel to discuss potentially sensitive information.

Written Expression. Following the second interview, participants were encouraged to complete a brief written exercise (see Appendix D) to gather information about their observations of overconforming behaviors by other athletes in their sport. Participants were asked at what point, and why, one might go too far to prove oneself as athlete, as well as whether they, themselves, had ever gone "too far" and, if so, why. Participants were then asked to identify, on a 5-point Likert-type scale how often they had observed each of five overconforming behaviors (i.e., playing while injured/in pain; disordered weight management, performance enhancing substance use, excessive violence, hazing). They were asked in what ways they had seen, or executed, each overconforming behavior. Where their written responses were unclear, participants were asked to elaborate on their responses; verbatim transcription of elaboration of written responses was included in transcripts for analysis. 


\section{Data Analysis and Interpretation}

Both deductive and inductive aims of the current study were met through the analysis of the data and presentation of the results. Analysis of the data commenced as soon as the researcher immersed herself in the data (Sparkes \& Smith, 2012). Two coding methods were employed for the current study. In the first, provisional coding was used to explore the contentcategorical (or specific, detailed; Leiblich, Tuval-Mashiach, \& Zilber, 1998) nature of the data. In the second, narrative analysis was used to explore the content-holistic (or broad) nature of the data (see Appendix B for more information about provisional and narrative coding). Throughout analysis, the researcher dove into the details of the story to establish and compare codes to previous literature and theoretical frameworks (i.e., provisional coding) and alternatively stepped back to examine the story, itself (i.e., narrative analysis). According to Smith and Sparkes (2009b), these methods “connect theory to data in a way that creates space for people's voices to be heard in a coherent context" that "provide compelling, detailed, and complex depictions of the psychological and social world" (p. 281). By using provisional and narrative coding frameworks, the essence of being human, with personal agency over self and, at the same time, being at the mercy of cultural forces, was honored and preserved instead of partitioned and controlled.

Results of the first stage of analysis (i.e., content-categorical) were presented as a word cloud (Wordle ${ }^{\mathrm{TM}}$; Feinberg, 2013) to depict how athletes define the "athlete" identity (i.e., research question one). Word clouds are visual depictions of word/concept frequencies. Specifically, words that appear more frequently are afforded larger font whereas words that are rarely recorded are given a smaller font. Doing so allows the reader to understand main themes quickly (McNaught \& Lam, 2010). Moreover, for the current study, words presented in green represented aspects of the athlete identity that were communicated to the researcher with positive 
regard whereas words presented in red were often referred to negatively; those represented in white were communicated positively by some and negatively by others.

The researcher then used creative nonfiction (Caulley, 2008; Smith, McGannon, \& Williams, 2015) to depict the content-holistic stage of data analysis. Creative nonfictions provide unique opportunities for dissemination to nontraditional readership given their accessible nature (Caulley, 2008). Notably, creative nonfictions are not representations of the data that serve to provide the "last word" of the studied phenomenon (Smith, McGannon, \& Williams, 2015). Rather, creative nonfictions encourage the reader to engage with the data, evoking emotional responses by focusing directly on participant experiences and encouraging varied interpretations to prompt continued dialogue and participation in a collective story (Smith \& Sparkes, 2014). Creative nonfictions are rooted in "fact," as communicated by participants, but presented as "fiction" to protect participants' privacy and move the reader (Smith, McGannon, \& Williams, 2015; Smith \& Sparkes, 2014). Because each piece of the narrative is not broken into its smallest part and discussed separately, it communicates theory and complex interactions while encouraging open interpretation (Smith, McGannon, \& Williams, 2015). See Smith, McGannon, \& Williams (2015) and Smith and Sparkes (2014) for more information regarding the advantages and considerations of using creative nonfiction in research contexts. For the present study, the creative nonfiction was presented in two parts. The first set the stage for the narrative, providing thick description of an important, defining event to orient the reader to what is coming (Caulley, 2008). In the second part, a monologue was used to present the data holistically, providing context and detail.

When constructing the creative nonfiction, three considerations were of importance: (a) preserving the structure of the narratives shared by participants, (b) preserving the voice of the 
narratives shared by participants, and (c) preserving the experiences highlighted in the narratives shared by participants. To address the first concern, the creative nonfiction was segmented into “chapters" created from the narrative structures presented by participants during their life story interviews. Moreover, analysis of the transcripts revealed a pattern of participant reporting such that each participant first shared a story or experience from his life in sport and followed that story by educating the researcher on how his behavior fit within the greater sport culture. This pattern was then preserved in the final creative nonfiction to reflect the structure, voice, and experiences of the narratives shared by participants. In an effort to preserve the voice of the participants, only language that affected the readability and flow of the overall story was altered. Otherwise, participants' own words and speech patterns were compiled to portray the participant voice. Finally, to preserve the nature of experiences shared by participants, episodes included in the narrative reflected accounts that were mentioned frequently or emphasized (e.g., tone/emotion), as well as those that were deemed important by the participants (e.g., turning points, vivid scenes). As such, the essence of each event was rooted in the participants' own experiences, reflecting each participants' associated thoughts, feelings, and behaviors.

To protect participant privacy and confidentiality, the creative nonfiction was presented from the perspective of one "character," who was created in light of the life stories and experiences of the three participants in the current study. It was important that this character reflect the level of identification with the athlete role, relative to other life roles, as expressed by the participants' AIMS scores. Further, the composite character's orientation toward seeking belonging and social recognition, gleaned from SMOSS scores, is highlighted throughout.

\section{Research Quality and Ethical Concerns}

Neither the ability to generalize results nor the discovery of absolute truth are aims of 
constructivist-interpretivist research (Ponterotto, 2005). Therefore, qualitative researchers have recommended that research should be judged on the basis of trustworthiness (Lublich, TuvalMashiach, \& Zilber, 1998; Yeh \& Inman, 2007). By shifting the focus to the evaluation of the researcher, human experience can be more accurately portrayed in research.

Support for the trustworthiness, or the "authenticity and consistency of interpretations grounded in data" (Yeh \& Inman, 2007), for the current study was met in a number of ways. Particularly, trustworthiness was established using written exercises for data triangulation, methods of self-reflexivity (i.e., researcher background statement, the use of a research team to analyze pilot data, use of a research assistant to analyze final sample data, analytic memos), and member checking. Following data collection, participants were asked if they would be willing to be contacted to review the researcher's interpretation of the data; all three participants agreed. Following data analysis, member checking by the participants was requested electronically on two occasions. Only one participant responded to requests for member checks. His only revisions included enlarging the size of the word, "sacrifice," in the word cloud as he believed it was paramount to the athlete identity and reducing the size of the words, "performance-enhancing substances," given his limited personal experience with them. Given that he was the only participant to respond, and the word cloud was a reflection of the collective definition of the athlete identity, no changes were made.

Recommended criteria to judge the quality of narrative research is grounded in (a) width, (b), coherence, (c), insightfulness, and (d) parsimony (Lublich, Tuval-Mashiach, \& Zilber, 1998, p.173). This criteria has been endorsed by other researchers in sport and exercise psychology who performed narrative, life story research (e.g., Carless \& Douglas, 2013a). For the current study, width was achieved by using various data from multiple sources. Coherence was 
addressed by condensing the data in a single, concise illustration of the athlete identity and corresponding story meant to represent the identity and experiences of the participants. The researcher strove for insightfulness by making the data accessible in illustration (i.e., word cloud) and narrative (i.e., creative nonfiction) forms that encourage the reader to reflect on his/her own experiences. Parsimony was sought throughout the presentation of results and interpretation of the data, with a balance of brevity and depth throughout.

Although the process of telling the life story can be significantly personal in nature, benefiting some individuals' self-exploration (Vajda, 2007), it can also feel invasive and may lead some participants to feel vulnerable. Consequently, the researcher, who holds a Master's degree in Counseling, offered resources to assist each participant with any potential adverse effects of participation in the study. Participants did not request, nor were they referred, to additional resources as a result of their participation in this study.

\section{Word Cloud}

\section{Results}

To address the first research question of the current study (i.e., to understand how wrestlers define the term, "athlete"), interview transcripts from three participants were provisionally coded for indications of perceived expectations placed on athletes, and resulting expected behavior. The results of that analysis, in the form of frequency of codes, were presented in a word cloud (see Figure 4, Appendix H) to provide a visual representation of the athlete identity, as defined by participants in the current study.

\section{Standardized Instruments}

AIMS. One participant ranked in the $90^{\text {th }}$ percentile of athlete identification compared to the AIMS normed population of male athletes (Cornelius \& Brewer, 2001); the remaining participants ranked in the $45^{\text {th }}$ and $37^{\text {th }}$ percentiles. Practically, these findings indicate that 
participants identified with the athlete role greater than $90 \%, 45 \%$, and $37 \%$ of their peers, respectively. Mean degree of identification with the athletic identity was in the $55^{\text {th }}$ percentile $(M$ $=40$ ). Therefore, the character created for the creative nonfiction was constructed to reflect a moderate degree of identification with the athlete role in comparison to other potential life roles.

SMOSS. To examine level of social motivation orientation in the present sample, data gleaned from the SMOSS was analyzed according to the subscales of Affiliation, Social Status, and Social Recognition (Allen, 2003). The most important social motivation orientation for the current sample was Affiliation $(M=4.29$, range $=3.57-4.86)$. Given the family atmosphere that was fostered in the wrestling environment, it is not surprising that these participants were highly motivated to belong. Social Status, on the other hand, was not a motivating factor $(M=2.33$, range $=1.5-3.25)$ for the sample. This may be because participation, alone, was not deemed a desirable motivation for engaging in the sport and humility was deemed necessary for gaining peer respect by participants in the study. Social Recognition motivation reported by the sample ranged from 1.25 to $4.5(M=3.25)$. Given the small sample size and range of responses, mean social recognition was not a good indicator of participant social recognition motivation. Specifically, two participants ranked social recognition motivation orientation above a 4 on a 5point Likert scale, whereas one participant ranked it at 1.25. This disparity in Social Recognition motivation is clearer when participant narratives are considered. That is, for two participants, high points in wrestling revolved mainly around success earned through hard work and sacrifice, especially if the task was deemed challenging or impossible by others; low points, for both participants, related mainly to failing to meet others' expectations of excellence and pervasive feelings of disappointing important others. The third participant's narrative revealed a discrepancy between faith and recognition. Specifically, he cited faith as his primary motivation, 
such that he should continue to compete given the gift he believed was bestowed upon him by a higher power and that emphasized the importance of humility, which minimized his desire to receive recognition from others as a measure of his success. However, he also spoke on several occasions about the positive feelings associated with recognition from his family, peers, and coaches. As a result, the character created for the creative nonfiction reflected a high motivation for affiliation and a moderately strong motivation for social recognition.

\section{Creative Nonfiction}

A creative nonfiction was written to address the second, third, and fourth research questions of the current study (i.e., evidence of acceptance as athlete, meaning of the athlete identity, behaviors performed in the athlete role). Data from tape-recorded and transcribed interviews, the AIMS, the SMOSS, and a written exercise were coded using provisional and narrative coding frameworks and compiled to create a comprehensive representation in the form of a life story that reflects the athlete identity and experiences with overconformity shared by the collegiate wrestlers who participated in the study. Collectively, the narratives, AIMS scores, and SMOSS scores indicated that the composite character created for the creative nonfiction have a moderate level of investment in the athlete role, as compared to other possible life roles, and that he should be motivated foremost by the desire to be a part of a family and gaining social recognition from the team. Moreover, he should remain humble and not seek external popularity as a result of his participation in sport. What follows is a representation of the life story and experiences with sport, as shared by the participants of this study.

\section{"Seven Minutes of Hell": The Life Story of a Wrestler}

I just finished one of the biggest tournaments of my young wrestling career. I wasn't very good-after all, I got a late start wrestling. My brother had been doing it much longer and was much better than me, but the agony of defeat was softened knowing that he and my dad would accompany me to Wendy's where we'd have our usual post-match-pigout. It's tradition to pig out after a meet, and I couldn't wait! Days and days of discipline were usually met with a win, but 
without that reward, the next best thing I could imagine was a full stomach.

We walk in the front doors of the restaurant. I am hit in the face with a wall of grease and condiments, meat and cheese. I instantly begin to salivate-I can actually taste a $1 / 2$ pound double with cheese! I can feel the anticipation building in my stomach and am suddenly, keenly aware of my intense hunger. I feel as if I haven't eaten in a year and it hurts-it really hurts. The pain makes me want to double over, but I will my feet forward. After all, I won't be eating if I can't tell the indifferent, gum-smacking cashier what I want.

I hurry to greet the woman picking at her fingernails. My brother orders, then my dad. Typical. I'm always the last to decide; although I didn't need the extra time tonight. My dad looks at me. "You're getting a salad." It wasn't a question.

Dad knew the sport well and wasn't pleased with my performance. It wasn't that I hadn't tried, I just made dumb mistakes and didn't work as hard going into the match as I could have. I think, "I'm getting a salad?" I'm furious. I convince myself, "He has to be kidding." If I thought my embarrassing loss or the wall of smells I ran into by stepping into this establishment and my sudden awareness of my hunger were punches to the gut, my father's command was a crippling blow.

Stunned, all I could muster was, "What, I'm getting a salad?!" As soon as I said the words, I waited for his retort. Would he be annoyed? Angry? Did I just lose my chance at any food for the night?

I wasn't sure what to expect, until he finally ended my anticipation. "Yeah, a salad. This is the day you're going to turn it around." My dad sent me a very clear message that night: you sacrifice for the match, and when it's over, you keep sacrificing.

\section{Chapter 1: Youth Sport}

I didn't grow up wrestling. When I was younger, I spent my time playing baseball. I played little league. It was fun and my dad really liked the sport. My family would come to games to cheer me on. Dad would take me to and from practices, which was nice because it was our way to spend time together.

The older I got, I tried different sports to see what I liked. I started wrestling a few years into elementary school. Wrestling in elementary school isn't tied to specific schools, so I enrolled onto a club wrestling team. There were a lot of guys around me who had been wrestling since they could walk - or at least that's how they talked about it. It didn't matter so much to me back then because wrestling was all about having fun. Our coaches would have us play dodgeball to keep it from getting too serious. My brother wrestled with me, so my dad learned a lot about the sport. It became our family activity. I was a quick learner. I didn't lose any matches my first year. I went twenty and zero. I tell everyone that was my only undefeated season. The trophies were sweet, and I enjoyed hanging out with my dad and brother, so we stuck with it.

In a year or two, my brother and I started going to open tournaments. We would travel around the state. The better I got, the more places we travelled. As I got older, youth state tournaments started taking up more of my time. It was really fun to see different places and learn the ropes. I would show up to these tournaments and the arenas were packed, just like high school state tournaments. I would look around to find ten mats laid out around the stadium and kids were wrestling on each one. The noise was deafening as families rooted for their kids. We were able to miss school for a few days to travel, which was a bonus.

\section{Chapter 2: Middle School Sport}


Middle school wrestling was different. I learned a lot about wrestling. I've seen my fair share of parents get thrown out of middle school gymnasiums. It actually gets pretty intense. My dad and brother were both really involved with my wrestling career, so they would work me out at night. They beat the shit out of me, plain and simple. It made me tough. It made me better. I learned that there will be suffering in wrestling, a lot of suffering, but I'm okay with that.

That mindset helped the further I went in wrestling. Wrestle-offs were a big deal, but it's also important to get the team points. So one week I'd wrestle up a weight class, then the next I'd have to cut weight and wrestle down a weight class. The first time I had to cut weight I cried. I hadn't ever done it before and liked to eat. At first, I tried to cut weight by exercising, but learned that exercising alone is rarely enough. Early on, I could cut one meal and make weight, but it only seemed to get harder after that.

I wrestled for both my school and my club team, so I kept going to youth state tournaments. Playing for both was time-consuming. One of the hardest decisions I've ever had to make was in $8^{\text {th }}$ grade when I had to choose between a school trip and youth states. Each year, everyone in the $8^{\text {th }}$ grade goes to the East coast to learn about history and see the sights and stay with friends in a hotel. It was something I had always looked forward to; it was a staple experience in the $8^{\text {th }}$ grade. When I made the youth state tournament, it was scheduled for the same weekend as the school trip. I chose the tournament, but it was hard. I had to go to school for a week without everyone else; there were only four students who didn't go on the trip. In wrestling you have to sacrifice, and sometimes the sacrifices are more social than physical.

\section{Chapter 3: High School Specialization}

I had to stop playing other sports in high school. I got a minor cervical spine injury in football. They took me off the field on a stretcher and everything - they take that stuff seriously. After that injury, my football coach called and said he wanted to talk to me and my parents. He knew how good I was getting at wrestling and that I probably wouldn't have the same career in football. He was pretty blunt. He just blurted out, "You can't put your future for wrestling at risk because you want to play high school football." It hurt to hear that football was going to be over, but he just kept saying, "You've worked so hard at wrestling, done it for so long. You have things to look ahead to. Focus there." I knew he was right. My parents knew he was right. That night was the end of football for me.

I stopped playing baseball that year, too. Wrestling and baseball overlap, so I missed the first three weeks of baseball season for the state wrestling tournament. By the time wrestling was over, the baseball team had conducted tryouts and been practicing together for a while. I didn't want to be that guy to come in late and have to play on junior varsity. I felt bad because my parents just bought me a new bat and glove for Christmas that year-which wasn't cheap — but I didn't want to be the young guy showing up a month into the season so I chose to just focus on wrestling year-round.

It paid off. I was district champion that year. It was a pretty big deal because my school hadn't had a district champion in a few decades, let alone a freshman district champion. I felt a rush as I stood on the podium and got my medal, the crowd cheering for me in the background.

In elementary and middle school, wrestling was a part of what I did, but in high school, it became the only thing I did. Wrestling was everything to me; it was literally my life! Coach would work us out at 6:30am. I'd go to class at 8:00am. I'd take weights, and wear full sweats through the whole class so I could lose a few pounds before lunch. Then I'd eat my lunch, finish classes, and head to practice in the afternoon. When I got home, I'd workout again. It was hard, but that's 
the thing about wrestling. It's all about hard work and commitment. We don't call injury time when we're on our backs, even if it hurts. Whether its practice or a match, it's better to leave it all out there and crawl off the mat. You'll be sorry if you don't, but you can't be mad at yourself if you do. Coach used to say, "It's seven minutes of Hell. If you throw up, you worked hard enough."

The next year, I developed several stress fractures in my back. I had to take a year off from wrestling. As hard as it was not to wrestle, I went to physical therapy four times a week and kept training on my own, three or four times a day. I knew if I wanted to win state championships by my senior year, I'd have to put in the work now.

By my senior year, I was ranked top 25 in the nation, but my rival was ranked number one. He had always been bigger than me, but we were in the same weight class my senior year. He was a two-time state champion. He hadn't lost to anyone in a few years. He was the only one I lost to all year long; six losses - that was it - and they were all to this guy. I had beat a couple of decent guys, so my coaches were happy, but I couldn't seem to beat him. Going into the state tournament, I remember thinking, "I have to beat this kid to win." I didn't.

\section{Chapter 4: College Recruitment}

The nice thing is that you can wrestle in college, even if you aren't a State Champ in high school. I started getting recruited before the state tournament even started. Coaches looked at me even when I lost matches because they saw the potential in me; they saw that I was hanging with guys who were more experienced and better than I was. They saw that I was willing to work and to suffer and to keep going no matter what. As long as I kept proving myself, I knew I'd get an offer.

There's a lot of hype behind committing. I got offers from several schools, and committed to a Division I school. I made myself one promise going in: if I start this, if I commit, I have to finish it. I knew it was a four-year thing. I decided the day I committed that I was committing to the work and the sacrifice for four more years. That promise has kept me going through college; it's important to me to finish what I start.

Once I committed, I didn't have to worry about where I was going like the guys who didn't get recruited had to. I could really just focus on my wrestling. Some of my teammates were freaking out. Some had offers to DII and DIII schools, but they were holding out for DI because it carries a certain status. Once I committed, and people knew I committed DI, I could relax some because everyone knew I wasn't just an athlete, I was a college athlete. For most of us, college is as far as we'll go in this sport, so it's important to make the most of it; I was fortunate to be able to do that-I worked to put myself in that position and it paid off.

\section{Chapter 5: College Wrestling}

There is a jump in intensity when you transition into high school, and another one when you transition into college. In college, you go from wrestling boys to wrestling men. Everything gets amplified; the stakes are higher, the team is more of a family, the things guys will do to succeed get more extreme. When I tore my shoulder, I had to sit out of practice, so I spent my time focusing on my fitness and doing what I could. Even after I got back to practice, my shoulder still slips out - I don't think the physical therapy really helped all that much-but I can move it so I don't have any complaints. I've always felt like I was the underdog and had something to prove. I also knew that I had the work ethic and dedication to do it, no matter what. There is always going to be a ceiling, or a wall. We push through it or jump over it. We have to break the barriers to get to the next level. We are taught that you have to push it to see what potential you have at the next 
level, and when you get there, you set your eyes on the next level and do what you have to.

The nice thing about wrestling is that, even though you're alone on the mat, you're never alone. Wrestling teams are family. It's thirty or forty guys in a room together, every day, beating the shit out of each other. Then, off the mat, we are brothers. Our coaches are father figures. We look up to them. We listen to them. They know what's best for us and we look out for each other. It makes you not want to go anywhere else. That feeling only gets stronger in college because you're away from your biological family, so you really count on each other that much more.

In wrestling, we practice 365; that's year-round. Coaches say, "It's a twelve month sport. If you want to do something with it, you can't just stick to one season and come back eight months later. You have to be practicing all year." So I practiced year-round, no matter what. I would work out on Christmas and other holidays because I just couldn't take a break. Participating doesn't make us athletes; it's all about dedication and showing that dedication.

It's amazing what some guys will do. In the upper weight classes, guys will take steroids in the summer to bulk up and then spend the year trying to lose fat. Other guys take all kinds of diet and weight loss pills. We learn from older guys on the team who have done it and see how they competed. Sometimes we look things up on the internet to find other ways. Some guys on my team looked up Epsom salt baths that open up your pores and make you sweat so you lose weight. I have never tried it, but they were willing to. I suppose it depends on the guy and what you consider "performance enhancing substances," but we all look for an edge where we can.

Once, to compete, I lost twenty-one pounds in three days. Coach brought me in on a Wednesday morning. The guy who started my weight class got hurt and coach wanted me to wrestle in his place that Friday. I was a freshman, so, without question, I said I could do it. I weighed in that day at practice and saw that I had twenty-one pounds to lose and practically no time to do it. I had cut weight before, but never like that. I had no idea how I was going to do it, just that the team would count on me for points. So I spent three days doing what I had to, to make weight. I didn't eat. I exercised in my sauna suit. I lived on that treadmill for three days. It was horrible. I was an absolute zombie. I was mentally broken. I literally could not walk up a flight of stairs without coming to tears. It was miserable.

So we got to the dual and their coach draws the weight class. I'm stoked, I'll be the last weight class of the day. I'm thinking, "Perfect. I can get some fluids in me. I'll be feeling good; I'll be ready to go." Both teams wrestled well - too well. It comes down to the last match and we're tied. Everyone else has wrestled, and it's up to me, the freshman, to win the dual. I know I'm tired, but I had the whole rest of the meet to rehydrate and sugar up, so I'm feeling good. I get on the mat, and I'm thinking, "I can beat this guy, this is my shot!" I take the kid down; I'm winning! Everyone on my team is cheering, and I feel on top of the world. By the end of the first period, I'm gassed. I can't even stand up. It's terrible. I tried so hard and literally could not move; I'm cramping everywhere! I ended up getting beat. We lost the match-my first match. It was my chance to prove myself and I blew it. I gave it every ounce that I had, and it wasn't good enough. I let my team down. I let my coaches down. The thing about wrestling, though, is that you just keep putting in the work. You know there will be suffering and you push through it.

\section{Chapter 6: Looking Back}

If you've never been involved in wrestling you won't understand it. There's no shape like wrestling shape. You can be in the best running shape of anyone you know, but after you wrestle for five minutes, if you're not in wrestling shape, you're puking. It's not like most sports where you have a goal, and you might come into contact with opponents standing in the way of achieving 
your goal. If you're trying to put a ball in the hoop, you might bump into an opponent, or he might swipe at the ball and accidentally hit you in the face. In wrestling, he's wrapping his arms around your neck to make you submit to him. When you're wrestling, you're putting your hands on your opponent to physically dominate the man in front of you-you're at war. Most people don't realize how brutal wrestling really is. It's a fight. It's the closest thing to a fight that is allowed in scholastic competition. Some guys lose their cool - they head butt or bite or put pressure on tight locks and holds. I've seen guys try to choke their opponents or tear shoulders out of sockets. When we're on the mat, we're fighting and when we're off the mat, we're not, and we don't hold grudges.

Most of us are willing to do anything to reach our goal. We will put our bodies through basically anything - arms and legs twisted, muscles torn, bones broken. Things heal. As long as they still work, we don't complain. It's all just so we can reach our goal. We want to train our minds to take a backseat so our bodies can do what they need to do. Nothing is too crazy. You can never go too far in wrestling. Just participating doesn't make you an athlete. We have to constantly train to be the best. We have to be willing to be comfortable being uncomfortable. We have to be willing to sacrifice a lot - energy, time, and food. We know we can cheat reps when Coach isn't looking, but in the long run, it doesn't hurt the coach, it hurts us. We don't gain anything from cheating, so why waste the time and effort? We have to be disciplined. It's just the nature of wrestling. It's something every wrestler has been taught since they started. Athletes have to dedicate themselves to a sport they love and believe in; we love and believe in wrestling.

We spend a lot of time learning to push our bodies past the limits, past what we think is possible. The first time I did a mile and a half buddy carry, I thought there was no way I'd be able to finish it. I did. I don't know how I did, but I did. Everything that's hard eventually comes to an end, and when you realize you did it, you find new potential and new limits to push. It's a really good feeling to look back and remember how hard a lift felt the first time I did it and how I never thought I'd survive some of the conditioning, but now it's easy and I look forward to doing it in the future because it feels like a break from the new hard stuff. It's important to keep the perspective. Let your body do the work - its only four or five short years - then you can deal with the repercussions.

Cutting weight is a source of pride, too. I always felt like, "I can do this and you can't. I'm mentally tough." I really liked being hungry because I thought, "I don't have to eat." It can get pretty miserable, but you can learn a lot about yourself from cutting weight. It teaches us that our bodies can do things we don't want to do. If we want to lose two more pounds, or ten, we absolutely can. As bad as it's going to hurt, it will be over eventually. It's purely mental. Some guys go two or three days without eating or drinking, wear sauna suits or neoprene scuba suits, and take weightloss pills-I'd say at least $85 \%$ of wrestlers wear plastics. We take that stuff seriously. It just takes discipline. I've told myself, "If I want to drink this pound of water, I have to lose a pound on the treadmill, first." I hold myself accountable and, for me, it's paid off.

\section{Chapter 7: Looking Forward}

I know wrestling won't last forever; most guys don't continue competing after college, and I've always known that. I don't want to leave college. I want to stay and represent my school as long as I can; I don't want to step into the real world. I know everyone has to eventually, I'm just not there yet.

After I graduate, I'll stay involved in wrestling, somehow. I don't know if I'll coach or I'll officiate, but I'll always be around wrestling. I've worked with kids during summer camps and that's been a blast, so I'll probably keep doing that. I'll work with the kids from my high school 
when I'm home and show them some things. I remember guys doing that for me and it really helped me to understand the sport and get better.

I want to give back to wrestling because it gave so much to me. It influenced my life. I'll always have the wrestler mindset to outwork people and do my best all the time. I'll honor my commitments and keep my promises. We learn to be disciplined and push boundaries and suffer so early on that we're equipped to handle life in any venue. Looking forward, I just want to stay active. I'll always look for ways to push the limits and find new potential. It may not always be in wrestling, but I'll find other ways to challenge myself.

Wrestling will always be a part of my life. I will always be a wrestler-that's how I will define myself when people ask. I'm proud to say that I'm a wrestler. Some people get it, and some people don't, but when you wrestle and you put in the work and you make the sacrifices and you prove yourself, it's a source of honor and I'll hold onto that forever.

\section{Discussion}

In an effort to better understand athletes' experiences with deviant overconformity, data from interviews and written assessments of three collegiate wrestlers were qualitatively analyzed using provisional and narrative coding frameworks. By presenting the results as a word cloud, the reader is provided a visual representation of the athlete identity, as described by athletes, themselves. By using a creative nonfiction, the participants' voices were given priority, allowing complex phenomena to be communicated, while conveying the ways in which participants' athletic identities were confirmed, ascribed meaning, and performed.

\section{General Findings}

The results largely support previous research on deviant overconformity, including the process by which it was learned (i.e., identity construction and confirmation, Donnelly \& Young, 1988), motivations that influenced uncritical acceptance (i.e., athlete identity, social motivation orientation; Hughes \& Coakley, 1991; Waldron \& Krane, 2005), and ways in which participants met sport norms (i.e., overconforming behaviors; Coakley, 2009; Hughes \& Coakley, 1991;

Waldron \& Kowalski, 2009; Waldron \& Krane, 2005). Moreover, the results extended previous theoretical work on athletes' identity formation and confirmation (i.e., socialization) process and introduce areas for further investigation. 
Overall, participants in this study reported "living" (Carless \& Douglas, 2013) the expectations they believed were placed on them, rather than "playing" or "resisting" those expectations. As a result of their commitment to embody the expectations they perceived of the athlete role year-round, in all contexts, participants in this study were accepted as athletes by their sportsnets (Nixon, 1993) and reinforced for their behaviors. Consequently, their perceptions of the athlete identity and expectations of overconformity as normative to the sport were reinforced, the meaning of the athlete identity was strengthened, and future overconforming behavior and motivation to continue involvement with the sport long-term was bolstered. Participants in the current study indicated that there was no point at which they considered their behaviors deviant. Instead, they communicated that because they were expected to push boundaries, there could be no limit — and thus, no way to go "too far" - in the pursuit of success.

Definition of the Athlete Identity. Overall, participants spoke positively about the athlete identity. They conveyed a sense of pride associated with the athlete identity, despite aspects of it that tended to invoke occasional negative thoughts or emotions. In general, participants in this study defined the athlete identity in terms of (a) the sport ethic, (b) behaviors to support adherence to the sport ethic, and (c) values learned as a result of athletic participation.

For instance, striving for distinction was a prominent aspect of the athlete identity. Efforts to demonstrate striving for distinction included disordered eating and disordered exercise behaviors that were believed to aid the participant in being successful in the future. In an effort to demonstrate adherence to the expectation that athletes sacrifice for The Game, participants discussed rigid workout schedules that made engaging in other life events difficult, including working out during holidays. Although they described many experiences of overtraining, unrelated to weight management, not one participant identified multiple daily workouts as 
deviant from the norm, nor did they identify those experiences as examples of "overtraining". Rather, multiple daily workouts, whether healthy or injured, represented expectations of the athlete role. Because participants valued playing through injury and pain, they discussed the importance of continuing participation, even when in considerable pain, especially in a match or when monitored by others. Given that participants believed that the ability to face and overcome barriers to athletic success was an important expectation of athletes, they believed that the use of diet pills, supplements, and, to a lesser extent, steroids, was expected of athletes.

Importantly, winning, itself, was not a central aspect of the athlete identity, according to participants in this study. Although previous research (e.g., Carless \& Douglas, 2013a; Waldron \& Krane, 2005) indicated that the message passed down through the sport culture reinforces the idea that "winning is everything," for participants in the current study, winning served to affirm athletes' commitment to the sport and confirmed their sense of athletic identity rather than serve as the ultimate goal of sport participation. Instead, as Coakley (2015) observed, winning is important to athletes given that it "symbolize[s] improvement and establishe[s] distinction,... ligitimize[s] their sacrifices, [gives] meaning to their overconformity, enable[s] them to continue playing... and generally insure[s] reaffirmation of the identity that [is] at the core of their selfconcepts" (p. 385). Notably, each participant regarded sport participation as fundamental in forming life lessons and values, including the importance of commitment, discipline, hard work, and family, which must then be demonstrated by the athlete.

Development of the Athlete Identity. Participants in the current study described a process of identity development and confirmation similar to that of Donnelly and Young (1988). Throughout each life story, participants described a process of presocialization to wrestling, whereby they learned about the sport from others outside of it, to an event that led them to seek 
involvement in sport (i.e., selection/recruitment), to a series of experiences that reflected their perceptions of the expectations of athletes (i.e., socialization), and finally to the recognition they received from others when they believed those expectations were met (i.e. acceptance/ ostracism). This process followed Donnelly and Young's (1988) identity construction and confirmation process well. Of note, participants introduced a cyclical nature to the identity construction and confirmation process such that one must continue to prove himself when there is a change in coaching staff, team dynamics, level of competition, injury, or change in weight class hierarchy in order to receive continued acceptance as "athlete”. Figure 5 (Appendix H) illustrates the process by which athletes in the current study constructed their athlete identities, were confirmed as athletes by their sportsnets, and continued the process of reconstruction and reconfirmation that continued through their college careers. The finding that identity construction and confirmation may be more cyclical than linear is important as it extends Donnelly and Young's (1988) work to reflect the continuous nature of identity (re)construction and (re)confirmation. In other words, it is on loan from others and dependent on relatively current actions and orientations.

Important socializing agents for participants in this study included fathers, brothers, coaches, and teammates. To a lesser extent, fans and media also provided validation for the athlete identity. Interestingly, coaches and teammates were likened to fathers and brothers later in the participants' wrestling careers, providing insight into how expectations to achieve the athlete status were communicated and the importance placed on becoming recognized as athletes. Confirmation of participants' athletic identity was mainly reported in social recognition of accomplishments (Waldron \& Krane, 2005). For example, one participant described the intense pride he felt in being recognized by his community for his status as a freshman district 
champion. Other participants cited instances where they drew large crowds, received praise from coaches, and respect from teammates as recognition of their status as athlete. By being given extra perks and trust from coaches, participants felt good about themselves. They were reinforced for the sacrifices they made that led to the distinction and recognition they received from their coaches and were validated in their efforts to prove themselves as athletes. These results indicate that enhancing self-esteem, proving masculinity, and continued participation were not only motivations (Coakley, 2015), but validation for deviant overconformity. Moreover, similar to research on hazing (e.g., Waldron, Lynn, \& Krane, 2011), participants discussed how athletes unwilling to distinguish themselves, sacrifice, and play through adversity were ostracized by coaches, as well as teammates, leading the participants in this study to identify overconformity as a condition of membership (Coakley, 2015) in the sport subculture.

Meaning of the Athlete Identity. Following analysis of the data from interviews, AIMS, SMOSS, and written expression, meaning of the athlete identity was expressed by the current participants of this study in two ways. First, participants expressed a sense of personal fulfillment as a result of their engagement in the athlete role. Moderate scores on the AIMS indicated that the athlete identity was meaningful to each of the participants, although to varying degrees. In interviews, participants explained that cutting weight represented mental toughness, overtraining represented discipline, and playing through injury represented sacrifice. Each participant expressed the importance of struggling and becoming stronger as a result of those struggles. If he was willing to do whatever was necessary, he believed he distinguished himself from his peers. Overall, perceiving oneself as mentally tough, disciplined, and strong was a source of pride for the participants given its positive effects on self-esteem and that it acted as proof of masculinity. Moreover, successfully engaging in overconforming behaviors meant that the participants 
could continue participating in the sport. Whereas one participant expressed his displeasure at the thought of retiring from the sport after its conclusion, another expressed that he would always consider himself an athlete, and, more importantly, a wrestler. Moreover, all three participants reported that they intended to continue their involvement with the sport throughout the duration of their lives (e.g., coach, referee, volunteer), further bolstering the level of importance their athlete identity and the sport seemed to hold for each. This serves as an important point for practitioners, given that deviant overconformity cannot be traced to a single motivating factor. Thus, practitioners must work to understand their athletes' individual motivations within the sport subculture in an effort to deter future overconforming behaviors.

Additionally, the strong family bond created in the wrestling team environment was an important aspect of the subculture, creating an in-group and an out-group. Particularly, similar to previous research (e.g., Atkinson, 2011), the strong social bond created in the sport subculture reinforced normative overconformity for participants in the study. Often, participants referenced recognition from others as significant in their lives. The greatest recognition expressed by participants was that for hard work, discipline and sacrifice, rather than wins or trophies. Paired with the regard the participants expressed for their fathers and brothers, as well as the coaches and teammates who eventually represented fathers and brothers within the wrestling family, the data suggested that the athlete identity was a meaningful role for these participants and that participation in the sport met deeply-valued needs for the participants. Moreover, participants often mentioned how individuals outside the wrestling subculture likely fail to understand its expectations, which prompted them to spend considerable time rationalizing their actions. Although the small sample size in the present study makes it impossible to generalize the results to the entire wrestling subculture, the participants' attempts to rationalize their behavior as 
normal, expected aspects of the wrestling culture indicated that normative overconformity, reinforced by the family atmosphere experienced by these participants, may have influenced their decisions to overconform.

Performance of the Athlete Identity. Participants in the current study supported Donnelly's (2004) proposition that athletes voluntarily engage in physical risks in an effort to confirm their identities as athletes and develop social bonds with sport stakeholders. For instance, even when injured and in significant pain during a match, one participant mentioned his refusal to call an injury timeout for fear he might be perceived as weak by those around him. Moreover, high points for the participants largely revolved around events where they perceived themselves as underdogs and needing to take risks in order to find success against the odds. Those risks included engagement in potentially dangerous weight management strategies, as well as behaviors that risked social isolation (i.e.., overtraining and limited social interaction with non-sport others).

Specific methods of overconformity discussed by participants largely supported previous literature, including disordered eating behaviors (e.g., Atkinson, 2011; Johns, 1998; Pettersson, Ekstrom, \& Berg, 2012), overtraining, management of chronic pain (e.g., Curry, 1993; Fenton \& Pitter, 2010), performance enhancing substance use (e.g., Waldron \& Krane, 2005), and intentional violence (e.g., Kowalski \& Waldron, 2010; Waldron \& Krane, 2005). Participants reported engaging in significant disordered eating (e.g., fasting for several days) and disordered exercise (e.g., several times a day in sauna suits) behaviors in an effort to distinguish themselves from their peers, prove their willingness to sacrifice, play through discomfort, and demonstrate an "I can" attitude, regardless of perceived barriers. Similar to Pettersson and colleagues' (2010) research, participants reported a sense of pride in their ability to master their bodies and push 
their limits, which symbolized their mental toughness. Participants also engaged in overtraining and underrecovery regularly to meet the demands placed on them, including wrestling yearround, several times a day in order to maintain "wrestling shape." Although it was not clear if chronic injury was a result of disordered eating/exercise behaviors and/or overtraining/ underrecovery, or merely the contact-nature of the sport, each participant discussed various injuries he sustained throughout his career. The greatest moments, shared with the most intense emotion and detail, related to triumphs over pain gained through pain tolerance, and, when necessary, medical intervention. Participants also referenced pain management practices observed in teammates and opponents, including repeated cortisone injections, daily NSAID overdoses, and bracing/taping, highlighting an important distinction between what participants believed to be substance use necessary for medical intervention and substance use for performance enhancement. Importantly, each participant discussed how pain was not an excuse to not perform well in wrestling and the importance placed on pushing through pain, regardless.

None of the three participants admitted using "performance enhancing substances" regularly in an effort to improve performance. However, two of the three spoke about teammates who had used steroids and/or diet pills to achieve higher levels of performance. Finally, participants in this study identified intentional violence as a necessary aspect of wrestling. They provided examples where they were "the boxer" as well as examples when they were "the punching bag." Given the close contact and "brutal" nature of the sport, violence was not only encouraged, it was seen as a necessary instrument to success in the sport. Similar to disordered eating, great pride was conveyed when discussing the violent nature of wrestling and the participants' various levels of success achieved given the "war"-like nature of the sport. Unlike Waldron and colleagues' (2011) research on hazing, it was rarely discussed by participants in the 
current study as an expectation of athletes in the wrestling culture. Instead, few references to hazing in the current study were related to participants' perceived discrepancies in treatment of the athletes on the team, such that athletes who were unwilling to work through pain and injury, or engage in extra workouts, were often ostracized by coaches and teammates, and were not characterized by the participants as "hazing". Moreover, participants in the current study introduced overtraining, which was not related to pain management, as an important behavior associated with uncritical acceptance to the sport ethic, requiring future inquiry.

Previous research has found that higher levels of athlete identity are related to performance strategies that may be hazardous to the athlete (e.g., Hale \& Waalkes, 1994). Therefore, it is somewhat surprising to find that only one participant highly identified as athlete, compared to his peers (Brewer \& Cornelius, 2001), given the pervasive use of potentially healthcompromising strategies by all three participants throughout their wrestling careers. Deviant overconformity was originally conceptualized as a rarity (Coakley, 2009), hence its classification as deviant, yet reported incidents of behaviors associated with overconformity in this sample appeared to well-surpass what might be expected of rare behavior. Combined with the finding that only one participant in the present study highly identified with the athlete role, despite all three discussing expectations of overconforming behaviors, as well as their engagement in those behaviors, these results indicate that overconforming may be considered normative, rather than an exception under certain conditions, by participants. However, future research is required.

It is important to note that a common theme through the data of all three participants was their perception that overconformity does not exist in wrestling. That is, if one is constantly striving to push the limits of what is possible, constraining methods to get there is counterintuitive. As one participant summarized, "Nothing is too crazy. You can never go too 
far. It's just the nature of wrestling." Thus, there was no perception of deviance when a wrestler expressed commitment to his sport and his team. This finding supports previously identified challenges to studying overconformity (e.g., Coakley, 2009). Consequently, instead of targeting a single point at which athlete behavior becomes deviant, perhaps researchers should investigate, and practitioners should address, levels or degrees of overconformity in athletes. Moreover, it begs the question of who is best-qualified to determine the appropriateness of athlete behaviors, and thus, the point at which athletes cross the line from normally-conforming, acceptable behavior to overconforming, unacceptable behavior: the athletes, themselves; policy makers charged with preserving the health and well-being of athletes; researchers who identify and conceptualize contributors to athlete behaviors that can then be used to address athlete wellbeing; and/or practitioners who work with athletes engaged in health-compromising behaviors. On one hand, are sport outsiders (e.g., researchers, practitioners), who have only second-hand knowledge of sport culture and expectations, restricting athlete behaviors on the basis of sampled norms and theoretical expectations? On the other hand, are sport stakeholders (e.g., athletes, coaches, administrators), who are more knowledgeable about sport-specific expectations, able to take a perspective that allows them to critically evaluate sport-specific expectations and take appropriate action? Although the answer is beyond the scope of the current study, it is an important point for future discourse.

Summary of Deductive Findings. Taken together, the data indicate that the participants in this study generally followed a process of identity formation and confirmation similar to that described by Donnelly and Young (1988). Moreover, the results support previous work by Hughes and Coakley (1991) which suggested that athletes learn the expectations of the sport (i.e., the sport ethic) during the socialization phase of identity development from individuals in 
their sportsnets (Nixon, 2004). Further, acceptance of sport expectations that prompted athletes to engage in overconforming behaviors was influenced by motivations to keep playing, prove masculinity, and enhance self-esteem (Coakley, 2009) as well as motivations to belong and be recognized for accomplishments (Waldron \& Krane, 2005). Finally, engagement in overconforming behaviors during the socialization stage led the participants to feel accepted by their peers as "athlete." See Figure 5, Appendix H for a conceptual framework of how athlete identity and overconformity were experienced by participants in the current study.

Summary of Inductive Findings. Interestingly, two important contributions to existing literature emerged from the data. First, participants in the current study provided evidence which suggested that athlete identity construction, as proposed by Donnelly and Young (1988), is more cyclical than linear. That is, even after perceiving acceptance from their sportsnets, participants still felt a need to continue to prove themselves. This may provide insight into the continued use of overconforming behaviors by athletes, even after they achieve an initial level of acceptance from those around them. Moreover, it may have implications for athlete retirement and transitions to different life roles, especially when retiring athletes lose access to sources of identity reaffirmation. However, further longitudinal research is necessary to better explain the cyclical nature of identity formation and its long-term consequences.

The acceptance participants felt from those in their sportsnets may have also contributed to their continued use of overconforming behaviors, despite having initially establishing themselves as athletes. Particularly for participants who scored higher on the AIMS and SMOSS, and those who detailed more significant events around efforts to keep playing, prove masculinity, be part of a family, and be recognized for their accomplishments, acceptance by others as "athlete" was motivation to continue to engage in the behaviors they perceived 
necessary to gain and maintain acceptance. Although this finding provides compelling significance to increase research efforts in the area of overconformity in sport, further research is necessary to determine if these findings are consistent with other athletes, in other contexts.

Second, the finding that participants engaged in a process of storytelling followed by rationalizing throughout the entirety of the interviews adds further interest to the findings of the current study. Each participant demonstrated a tendency to first present a story to signify an important time in their lives, then explained their behaviors in the context of the sport (wrestling) subculture. That tendency was reflected in the final creative nonfiction where each experience is followed briefly by education about how that behavior fits within the greater sport (wrestling) subculture. Possibly, the fact that the researcher was an outsider to the wrestling culture contributed to the pattern of rationalizing demonstrated by participants. That is, participants in life story research select the stories they share on the basis of what they want the audience (e.g., the researcher) to know and what they do not want the audience to know in an effort to convey a particular identity (McAdams, 1998). Moreover, some life story researchers have explained the implications of over-sharing and efforts to re-shape the audience's interpretations of the participant's identity (e.g., Vajda, 2007). Consequently, it is not surprising that participants in the current study found themselves in a cycle of disclosing a behavior that, to individuals outside of the sport (wrestling) subculture, may be deemed inappropriate, and seeking to educate the lay audience about why their behaviors were appropriate given the context.

Alternatively, although defense mechanisms, including rationalizing, are expected in life story research (see McAdams, 1998), the recurrent pattern demonstrated by participants in this study may indicate an important process in identity development. In previous work with overconforming rhythmic gymnasts, one gymnast discussed how she knew her disordered eating 
(i.e., overconforming) behaviors were wrong in the non-sport culture, but that she engaged, regardless, given the positive feelings she experienced when encouraged by her coaches (Johns, 1998). Perhaps rationalizing was used by the participants in the present sample in an effort to resolve cognitive dissonance whereby they knew their behaviors were considered deviant to the larger, non-sport population, yet chose to engage in them, regardless, in an effort to find what they perceived as success and acceptance in the sport culture. Future research to examine defense mechanisms, particularly rationalizing, when disclosing overconforming behaviors is of great importance to future research on deviant overconformity.

\section{Limitations}

Possible limitations for the current study included: (a) researcher biases that affected interpretation, (b) inability to verify the accuracy of participant experiences, and (c) possible confounding effect of varied participant sport histories. Efforts to illuminate researcher bias included the use of a research team, self-reflexive techniques (e.g., researcher statement, analytic memos, creative nonfiction), and member checking. However, according to the constructivistinterpretivist paradigm that guided the design of this study, it is impossible to separate the researcher from the study (Ponterotto, 2005). The mere act of participating in the research process and the researcher's position as an outsider to the culture likely affected participant definitions of identity and disclosures of deviant over conformity. As such, and consistent with the constructivist-interpretivist paradigm, instead of striving to eliminate researcher bias, the researcher strove to co-construct the meaning of the athlete identity and behaviors associated with it, alongside the participants.

Given the sensitivity of the variables of interest in this study, it was possible that the researcher would experience resistance or defensiveness from the participants. According to 
McAdams (1998), defenses serve as "narrative strategies" (p. 1127) employed to maintain coherence in the story and/or conceal stories the narrator does not want told. Typical defenses found in previous life story research include: denial, projection, intellectualization, rationalization, isolation, and identification (McAdams, 1998). The use of these defenses in the life stories of the participants in the current study was not the focus of the study, although several were observed during analysis of the results. Therefore, whether the participants used defenses that alter the "truth" about their identities or experiences is moot. What mattered was how participants constructed their identities and interpreted their experiences related to the athlete identity; whether those moments and experiences were grounded in some external truth was irrelevant.

The distinction between "athlete" identity and "wrestler" identity was difficult to discern in this study. Specifically, given that all three participants in the current study specialized in wrestling at some point in high school, and only competed in wrestling in college, it would be logical to assume that the identity they defined would be largely biased by the wrestling subculture. However, all three also competed in other sports from youth sport leading up to their specialization in high school. Given that existing research has not yet pinpointed the specific time during which athletes (a) solidify their definition of the athlete identity and (b) begin engaging in overconforming behaviors, it is impossible to know whether the participants in the current study were defining the "wrestler" identity, specifically, or the "athlete" identity, more broadly. Future research that investigates and compares athlete identity and deviant overconformity in other sport populations (e.g., other sports, other regions, other nationalities, other competitive levels) would be a valuable step in determining the extent to which sportspecific subculture affects definitions athletes' identities and perceptions of sport expectations. 
Moreover, more specific research to specify the point at which athletes are socialized to overconform (e.g., developmental level, competitive level), especially given the cyclical nature of socialization that emerged from the current study, may help to untangle the contribution of specific sports, or broader sport cultures, to athlete decisions to overconform.

\section{Strengths}

The current study presents a number of unique strengths. Methodologically, the current study used an under-utilized means of data collection in exercise and sport science: life story interviewing. Although some researchers (e.g., Carless \& Douglas, 2013a) have used life story narrative methods in sport and exercise psychology, it is historically rare. Moreover, creative nonfiction and word clouds are underutilized data representation tools in sport and exercise psychology. By using both methods in the current study, the reader was encouraged to connect with the data in a way that is unique from existing methods of data presentation.

Additionally, athlete identity and deviant overconformity are not revolutionary concepts in sport and exercise psychology, and are often taken-for-granted by sport stakeholders. However, multiple theoretical frameworks were combined in the current study in an effort to connect existing and future literature in a comprehensive way. Moreover, this study introduced several points for future theoretical and basic research. Identifying the potentially cyclical nature of (a) athlete identity (re)construction and (re)confirmation, as well as (b) the potentially cyclical reinforcement of overconforming behaviors and acceptance provides new areas of inquiry.

Similarly, new areas of inquiry related to deviant overconformity include the role rationalization plays in the lives of overconforming athletes. Specifically, it was not clear in the present study if rationalization was the result of (a) the researcher as an outsider to the wrestling subculture, and/or (b) cognitive dissonance stemming from the perception that although 
overconforming behaviors were perceived as acceptable in the wrestling subculture, they were perceived as deviant from behaviors in the larger society. Although one cannot definitively draw the conclusion that participants in the current study rationalized their overconforming behaviors within the context of the sport subculture in an effort to resolve cognitive dissonance that resulted from engaging in behaviors they knew were "wrong" (Johns, 1998) in the non-sport culture, their repeated patterns of reporting and rationalizing indicate that future research in this area is necessary.

\section{Practical Implications}

Practically, it is important to note the extensive use of overconforming behaviors reported by participants in the current. Specifically, one participant estimated that $85 \%$ of collegiate wrestlers utilize sauna and/or rubber suits for weight management. Overall, participants in the current study indicated a long-standing tradition of sauna suit use in many levels of competition, encouraged by some coaches and peers. This supports Gibbs and colleagues' (2009) assertion that NCAA guidelines regarding weight management have only been partially effective. Given that using sauna and/or rubber suits is prohibited by NCAA policies (Gibbs, Pickerman, \& Sekiya, 2009), future research regarding best practices to discourage the potentially dangerous use of sauna and/or rubber suits for weight management is necessary.

Relatedly, an important implication for practitioners stems from the finding that participants engaged in multiple overconforming behaviors. Specifically, by understanding deviant overconformity as a possible undercurrent for athletes engaged in health-compromising behaviors, practitioners can assess for level of overconformity and, as necessary, screen for additional health-compromising behaviors that may otherwise go unnoticed and untreated. Additionally, it is important to teach athletes who have previously overconformed to the 
sport ethic to manage the pressures of sport, such that they feel competent to balance meeting sport expectations within healthy parameters. Doing so would require practitioner assessment of level of conformity to sport norms, which may be aided by future research efforts to create an instrument to measure deviant overconformity. If a client is believed to overconform to sport norms, and see those behaviors as normative, then he may be more likely to revert to those behaviors when returned to the sport environment. Therefore it is important to teach him skills to manage those expectations without resorting to health-compromising behaviors.

Moreover, participants in the current study outlined a process of understanding the athlete identity that started when they were young. This developmental process, and the significance of their experiences in sport, intensified over time, leading the participants in this study to engage in overconforming behaviors with increased frequency and severity over time. Given that overconforming behaviors were seen as normative expectations by participants in this study, and subsequently that "you can never go too far in wrestling," prevention efforts for behaviors associated with overconformity should include elements aimed at (a) educating young athletes, as well as individuals in their sportsnets and (b) teaching athletes to identify and navigate appropriate boundaries that protect their long-term health and well-being as a result of their sport participation. 


\section{References}

Allan, E.J. \& Madden, M. (2008). Hazing in view: College students at risk. Initial Findings from the National Study of Student Hazing. Retrieved from http://www.stophazing.org/wpcontent/uploads/2014/06/hazing_in_view_web1.pdf.

Allen, J.B. (2003). Social motivation in youth sport. Journal of Sport \& Exercise Psychology, 25, $551-567$.

Andersen, T. \& Finucane, M. (2015). Aaron Hernandez found guilty of murder: Convicted in 2013 shooting death of Odin Lloyd, sentenced to life in prison. The Boston Globe. Retrieved from http://www.bostonglobe.com/metro/2015/04/15/hernandez-jurorsdeliberate-for-seventh-day/QUaFD2uTTYn65GsKTEY26O/story.html

Atkinson, M. (2011). Male athletes and the cult(ure) of thinness in sport. Deviant Behavior, 32, 224-256.

Bracken, N.M. (2012). Substance use: National study of substance use trends among NCAA college student-athletes. Indianapolis, IN: The National Collegiate Athletic Association.

Brewer, B.W., \& Cornelius, A.E. (2001). Norms and factorial invariance of the Athletic Identity Measurement Scale. Academic Athletic Journal, 16, 103-113.

Buckner, C. (2014). As 'Malice at the Palace" brawl turns 10, impact lasts. USA Today. Retrieved from http://www.usatoday.com/story/sports/nba/pacers/2014/11/16/pistonsmalice-palace-brawl-commissioner-artest-adam-silver/19138239/

Carless, D. \& Douglas, K. (2013a). Living, resisting, and playing the part of athlete: Narrative tensions in elite sport. Psychology of Sport and Exercise, 14, 701-708.

Carless, D. \& Douglas, K. (2013b). "In the boat" but "selling myself short": Stories, narratives and identity development in elite sport. The Sport Psychologist, 27, 27-39. 
Caulley, D.N. (2008). Making qualitative research reports less boring: The techniques of writing creative nonfiction. Qualitative Inquiry, 14(3), 424-449.

Coakley, J. (2009). Deviance in sports: Is it out of control? In J. Coakley (Ed.) Sports in Society: Issues and Controversies, pp. 152-193. New York, NY: McGraw-Hill.

Coakley, J. (2015). Drug use and deviant overconformity: A sociological approach. In J. Hoberman, I. Waddington, \& Moller, V. (Eds.) The Routledge Handbook of Drugs and Sport, pp. 379-392. London, UK: Routledge.

Cottler, L.B., Abdallah, A.B., Cummings, S.M., Barr, J., Banks, R., \& Forchheimer, R. (2011). Injury, pain, and prescription opioid use among former National Football League (NFL) players. Drug and Alcohol Dependence, 116, 188-194.

Creswell, J.W. (2007). Qualitative Inquiry and Research Design: Choosing Among Five Approaches ( $\left.2^{\text {nd }} e d\right)$. Thousand Oaks, CA: SAGE Publications, Inc.

Creswell, J.W. (2009). Research Design: Qualitative, Quantitative, and Mixed Methods Approaches $\left(3^{\text {rd }}\right.$ ed). Thousand Oaks, CA: SAGE Publications, Inc.

Curry, T.J. (1993). A little pain never hurt anyone: Athletic career socialization and the normalization of sports injury. Symbolic Interaction, 16(3), 273-290.

Donnelly, P. (2004). Sport and risk culture. In K. Young (Ed.) Sporting Bodies, Damaged Selves: Sociological Studies of Sports-Related Injury, pp. 29-58. San Diego, CA: ELSEVIER, Inc.

Donnelly, P. \& Young, K. (1988). The construction and confirmation of identity in sport subcultures. Sociology of Sport Journal, 5, 223-240.

Dunn, M., Thomas, J.O., Swift, W., \& Burns, L. (2011). Elite athletes' estimates of the prevalence of illicit drug use: Evidence for the false consensus effect. Drug and Alcohol 
Review, 31, 27-32.

Feinberg, J. (2013). Wordle ${ }^{\mathrm{TM}}$. Retrieved from www.wordle.net on March 13, 2015.

Fenton, L.T. \& Pitter, R. (2010). Keeping the body in play: Pain, injury, and socialization in male rugby. Research Quarterly for Exercise and Sport, 81(2), 212-223.

Ganim, S. \& Sayers, D. (2014). UNC report finds 18 years of academic fraud to keep athletes playing. CNN Investigations. Retrieved from http://www.cnn.com/2014/10/22/us/uncreport-academic-fraud/

Gibbs, A.E., Pickerman, J., \& Sekiya, J.K. (2009). Weight management in amateur wrestling. Sports Health, 1(3), 227-230.

Hale, B.D. \& Waalkes, D. (1994). Athletic identity, gender, self esteem, academic importance, and drug use: A further validation of the AIMS. [Abstract] Journal of Sport \& Exercise Psychology, 16(S), S62.

Hoover, N.C. (1999). National survey: Initiation rites and athletics for NCAA sports teams. Available from http://www.alfred.edu/sports_hazing/docs/hazing.pdf.

Hughes, R. \& Coakley, J. (1991). Positive deviance among athletes: The implications of overconformity to the sport ethic. Sociology of Sport Journal, 8, 307-325.

Johns, D. (1998). Fasting and feasting: Paradoxes of the sport ethic. Sociology of Sport Journal, $15,41-63$.

King, S., Carey, R.S., Jinnah, N., Milington, R., Phillipson, A., Prouse, C., \& Ventresca, M. (2014). When is a drug not a drug? Troubling silences and unsettling painkillers in the National Football League. Sociology of Sport Journal, 31, 249-266.

Lieblich, A., Tuval-Mashiach, R., \& Zilber, T. (1998). Narrative Research: Reading, Analysis, and Interpretation. Thousand Oaks, CA: SAGE Publications, Inc. 
Marshall, C. \& Rossman, G.B. (2006). Designing Qualitative Research. Thousand Oaks, CA: SAGE Publications, Inc.

McAdams, D.P. (1998). The role of defense in the life story. Journal of Personality, 66(6), 11251146.

McAdams, D.P. (2001). The psychology of life stories. Review of General Psychology, 5(2), $100-122$.

McAdams, D.P. \& Guo, J. (2014). How shall I live? Constructing a life story in the college years. New Directions for Higher Education, 166, 15-23.

McNaught, C. \& Lam, P. (2010). Using Wordle as a supplementary research tool. The Qualitative Report, 15(3), 630-643.

Nixon, H.L. (1992). A social network analysis of influences on athletes to play with pain and injuries. Journal of Sport and Social Issues, 16, 127-135.

Nixon, H.L. (1993). Accepting the risks of pain and injury in sport: Mediated cultural influences on playing hurt. Sociology of Sport Journal, 10, 183-196.

Nixon, H.L. (2004). Cultural, structural and status dimensions of pain and injury experiences in sport. In K. Young (Ed.), Sporting Bodies, Damaged Selves: Sociological Studies of Sports-Related Injuries (pp. 81-97). Oxford, UK: Elsevier, Ltd.

Peacock, J.L. \& Holland, D.C. (1993). The narrated self: Life stories in process. Ethos, 21(4), 367-383.

Petrie, T.A., Greenleaf, C., Reel, J. \& Carter, J. (2008). Prevalence of eating disorders and disordered eating behaviors among male collegiate athletes. Psychology of Men \& Masculinity, 9(4), 267-277.

Pettersson, S., Ekstrom, M.P., \& Berg, C.M. (2013). Practices of weight regulation among elite 
athletes in combat sports: A matter of mental advantage? Journal of Athletic Training, 48(1), 99-108.

Ponterotto, J.G. (2005) Qualitative research in counseling psychology: A primer on research paradigms and philosophy of science. Journal of Counseling Psychology, 52(2) 126-136.

Rexroat, M. (2014). NCAA national study of substance use habits of college student athletes: Final report. Retrieved from http://www.ncaa.org/sites/default/files/Substance\%20Use\% 20Final\%20Report_FINAL.pdf.

Smith, B. \& Sparkes, A.C. (2009a). Narrative inquiry in sport and exercise psychology: What can it mean and why might we do it? Psychology of Sport and Exercise, 10, 1-11.

Smith, B. \& Sparkes, A.C. (2009b). Narrative analysis and sport and exercise psychology: Understanding lives in diverse ways. Psychology of Sport and Exercise, 10, 279-288.

Smith, B., McGannon, K.R. \& Williams, T. (2015). Ethnographic creative non-fiction: Exploring the what's, why's and how's. In L. Purdy and G. Molner (Eds.), Ethnographies in Sport and Exercise (pp. ). London : Routledge.

Sparkes, A.C. \& Smith, B. (2012). Narrative analysis as embodied engagement with the lives of others. In J.A. Holstein and Gubrium, J.F. (Eds.), Varieties in Narrative Analysis. Los Angeles, CA: SAGE Publications, Inc.

Sparkes, A.C. \& Smith, B. (2014). Qualitative Research Methods in Sport, Exercise and Health: From Process to Product. New York, NY: Routledge.

Vajda, J. (2007). Two survivor cases: Therapeutic effect as a side product of the biographical narrative interview. Journal of Social Work Practice, 21(1), p. 89-102.

Vetter, R.E. \& Symonds, M.L. (2010). Correlations between injury, training intensity, and physical and mental exhaustion among college athletes. Journal of Strength \& 
Conditioning Research, 24(3), 587-596.

Waldron, J.J. \& Krane, V. (2005). Whatever it takes: Health compromising behaviors in female athletes. QUEST, 57, 315-329.

Waldron, J.J. \& Kowalski, C.L. (2009). Crossing the line: Rites of passage, team aspects, and ambiguity of hazing. Research Quarterly for Exercise and Sport, 80(2), 291-302.

Waldron, J.J., Lynn, Q., \& Krane, V. (2011). Duct tape, icy hot \& paddles: Narratives of initiation onto US male sport teams. Sport, Education and Society, 16(1), 111-125.

Yeh, C.J. \& Inman, A.G. (2007). Qualitative data analysis and interpretation in counseling psychology: Strategies for best practices. The Counseling Psychologist, 35(3), 369-403.

Young, K. (1993). Violence, risk, and liability in male sports culture. Sociology of Sport Journal, 10, 373-396.

Young, K., McTeer, W. \& White, P. (1994). Body talk: Male athletes reflect on sport, injury, and pain. Sociology of Sport Journal, 11, 175-194. 
APPENDIX A 


\section{Literature Review}

Given the sparse literature on the explicit study of deviance in sport, as framed by sociological constructs of deviance, and especially the study of overconformity in sport (Coakley, 2015), it is necessary to first understand the larger framework of deviance in modern society. Once a common understanding of deviance in sport, specifically overconformity, is considered, understanding the process by which athletes overconform and the behaviors indicative of that overconformity can follow. Such is the aim of this review of literature.

\section{Deviance}

In their original work, Hughes and Coakley (1991) became interested in overconformity to the sport ethic given that "conforming to the norms of the sport ethic was expected, and overconformity was seen as setting athletes apart as exemplars" (Coakley, 2015, p. 6). However, previous literature focused on negative deviance, or those behaviors which were the result of a rejection of societal norms (i.e., underconformity). Overconformity, or positive deviance, on the other hand, was not readily accepted and discussed in sociological literature. In fact, it was hotly debated (Ben-Yehuda, 1990; Sagarin, 1985). This debate, unfortunately, adds difficulty to the study of overconformity in athletes given that it has contributed to the dearth of research available in sociological disciplines, let alone in the sport context.

As defined by sociologists, deviance encompasses "rule-breaking and other nonconformity which is viewed in the society in a negative way, and hence, reacted to with scorn, hostility, punishment, or an effort to effectuate change" (Sagarin, 1985, p. 169). From this perspective, deviance has a negative connotation, as a term reserved for individuals who disregard societal norms and expectations. Therefore, significant debate exists in the wider sociological literature as to the appropriateness of creating a category of "deviance" whereby 
individuals conform to the norms and expectations of a society or culture to the point of abnormality (Ben-Yehuda, 1990). This perspective reflects an absolutist view of deviance (Coakley, 2009), such that a single line of unacceptable behavior exists and that individuals who cross that line should be negatively evaluated and labeled "deviant." Scholars who endorse the absolutist approach to deviance have determined that "positive" deviance is inaccurate in that it does not share the same negative sanctions implicit in the traditional view of deviance (e.g., Sagarin, 1985). According to one such scholar, endorsing "positive" deviance would "collapse into one group, two ends of continua that have nothing in common except that they do not meet in the middle" (p. 169). It seems, however, that a portion of the debate so passionately demonstrated by some is founded more in a violation of language (Ben-Yehuda, 1990) of categories created and agreed upon years ago, than in the idea that some uncritically accept norms to an extent greater than other, normally conforming, individuals do. For example, according to Sagarin (1985)

It is difficult to believe that anyone mingling in the English speaking communities, at least in America and England, is using the term "deviant" in a manner that differs markedly from the definitions of scientists, and particularly to locate anyone using it in a manner applying to all those who differ from the norm or average, whether in a creditable or discreditable direction (p. 174).

Specifically, Sagarin (1985) argued, the term "positive deviance" is an error discerning between "deviance" and "deviation." Accordingly, he stated that deviance is a negative departure from normal whereas deviation is a departure from normal in either direction, whether perceived positively or negatively by larger society. Opponents to this perspective, however, argue that deviance, as defined in the social sciences, is unique from deviance in other sciences, in that it makes no reference to the negative valance of deviance (e.g., Ben-Yehuda, 1990).

Consequently, a second view of deviance, the constructionist view, is characterized as a 
dynamic view of conformity whereby individual behavior is evaluated along a range of normal behavior that is negotiated by the norms of that culture (Coakley, 2009). According to this perspective, deviance is "any act, role/career, attribute or appearance that departs significantly from social situational expectations... [S]ome deviance is appraised as violating situational expectations and is termed negative deviance while other deviance is viewed as surpassing social situational expectations and is called positive deviance" (Dodge, 1985, p. 22). Therefore, deviant behavior is any behavior that falls outside of the normal range, on either the underconforming (i.e., negative deviance) end of the spectrum or on the overconforming (i.e., positive deviance) end of the spectrum. According to constructionists, deviance is negotiated as individuals determine the range of acceptable behavior on the basis of their values, test the boundaries of acceptable behaviors, and challenge power dynamics as boundaries are tested (Coakley, 2009). As a result, most social artifacts (i.e., ideas, traits, actions) are considered normal and acceptable within the culture. At the extremes, underconformity may lead to lawlessness and widespread anarchy where overconformity may lead to unquestioning acceptance of norms and, resultantly, fascism. Therefore, both types of deviance are considered abnormal in that they are a departure from the normal, and both may lead to dangerous consequences.

As a final note on deviance in the larger sociological context, some researchers have argued that the constructionist view of deviance, to include over- and under-conformity, can be further broken down based on the acceptability of social artifacts (i.e., ideas, traits actions). Specifically, Heckert and Heckert (2002) proposed a 2x2 taxonomy for classifying deviance that examines both the extent to which rules are followed or broken (i.e., normative expectations) and the evaluation potential of the deviant behavior (i.e., social reactions and collective evaluations; see Figure 6, Appendix H). As a result, their typology included (a) positive deviance (i.e., 
socially acceptable overconformity), (b) rate-busting (i.e., socially unacceptable overconformity), (c) deviance admiration (i.e., socially acceptable underconformity), and (d) negative deviance (socially unacceptable underconformity).

Applied to sport, examples of each include: (a) the use of routine NSAID injections to manage pain and continue playing as a form of positive deviance, (b) steroid use to break records as a form of rate busting, (c) pitcher retaliation for the seemingly intentional targeting of his teammate in a previous play as deviance admiration, and (d) hiring someone to break an opponent's leg as negative deviance. In relation to the sport ethic, receiving NSAID injections to manage pain and continue playing despite chronic discomfort is a demonstration of one's commitment to the sport, to playing through pain and injury, and is therefore respected by other sport stakeholders. However, taking steroids places the recipient at an unfair advantage, and, thus although done to overconform to the norm of striving for distinction, is socially unacceptable and termed rate-busting. Although Heckert and Heckert's (2002) deviance taxonomy may have great utility in understanding athlete behavior, it has yet to be tested in the literature. Consequently, while it may prove valuable to explaining the results of the present study, a priori emphasis will be placed on understanding general overconformity in athletics as opposed to distinguishing between positive deviance and rate busting during data collection and analysis.

\section{Overconformity in Sport}

The willingness of the athlete to participate in behaviors condoned by the subculture of sport is how many athletes exhibit their commitment to the sport ethic. The sport ethic, as proposed by Hughes and Coakley (1991) was developed following three years' worth of informal conversations between the authors and athletes, coaches, team trainers, and physicians at the amateur and professional levels. These conversations were combined with existing 
research on high performance sport to better understand the structure of elite sport and explain athletes' willingness to risk their bodies in pursuit of the athlete identity (Coakley, 2015). The definition, recognition, and evaluation of athletic identity, as manifested by the athletes' actions, as well as the definition, recognition, and evaluation of the sport structure, are then guided by how they is compare to the ideal: adherence to the sport ethic.

The norms of the sport ethic include: (1) dedication to The Game, (2) striving for distinction, (3) accepting risks and playing through pain, and (4) overcoming all obstacles in the pursuit of possibilities (Coakley, 2015; Hughes \& Coakley, 1991). As a reflection of the first expectation of the sport ethic, athletes should sacrifice other areas of their lives to pursue sport excellence. Athletes are expected to prioritize the game above all other activities, relationships, and identities in order to demonstrate to other athletes, sports staff, and the world, that they are truly dedicated to the sport, above all else. Athletes who elect to homeschool instead of attend public schools, in favor of increased training time, and that may experience withdrawal from same-aged peer groups as a result, thus, exhibit commitment to the norm of sacrificing for the game. Likewise, families who choose to postpone family vacations in favor of travel tournaments demonstrate their willingness to sacrifice the value of family time for the value of winning and distinction.

In an effort to strive for distinction, athletes are expected to demonstrate constant improvement. They are expected to seek the perfect performance, and, once each benchmark is reached, continue reaching for the next. In popular sport conversations, fans, coaches, and peerathletes discuss how "real" athletes are "hungry." That is to say that they are never satisfied with previous performances, no matter how ground-breaking they are. For instance, Nick Saban, after winning his second consecutive national college football championship in 2013, stated that 
although he appreciated what his team did to win, his returning athletes had little time to celebrate their accomplishment because it was time to move onto the next season, and, presumably, another national championship (Gould, 2013).

Accepting risks and playing through pain is best demonstrated by the many athletes who push their physical limits, accepting the risks of unregulated performance-enhancing substances or unhealthy eating practices, as well as competing while injured. This expectation is readily identified in cases like Kerri Strug, who was praised openly by the media for performing a second vault in the 1996 Olympics in Atlanta, GA, despite two torn ligaments in her ankle sustained on her first vault (Weinberg, n.d.) and Barrett Jones, who played in the 2013 BCS National Championship on torn ligaments in his foot (Gould, 2013). Perhaps less wellremembered is Brett Favre's admitted addiction to Vicodin in the mid 1990's (King, et al, 2014), an unfortunate result of his desire to play through chronic and debilitating pain. Perhaps less well-known, was Heidi Guenther who demonstrated her dedication to ballet by losing weight at her choreographer's request, regardless that she did so by severely disordered eating practices that eventually killed her two years later at 93 pounds (Baker, 1999; Hewitt, 1997). Although the ballet company she danced for stated openly that they had recently urged her to regain some of her weight, she received more roles and complements the thinner she became, reinforcing the norm that athletes who play through pain and injury are rewarded for their efforts. After all, ballet is "a fiercely competitive world in which physical perfection can often seem like the expected norm, rather than an unattainable ideal" (Hewitt, 1997, n.p.).

Finally, regardless of athletes' efforts to demonstrate their dedication through sacrifice, continual striving for perfection, and willingness to endure pain and fear without backing down, obstacles are a necessary part of sport. At best, in the spirit of equal competition, on a single 
occasion of one-on-one competition, athletes and teams have a 50\% chance of success in competition. Considering that many more athletes may compete in a single competition (e.g., track and field, swimming) and that many more teams may compete for a single title (e.g., national basketball title), the $50 \%$ chance of success may drop to less than $1 \%$. For instance, at the beginning of the academic year, one could argue that 128 of Division I-A football teams compete for a national title; however, only one will earn that title come playoffs. Additionally, the opposing team/athlete is not the exclusive obstacle athletes face. Yet, the norm of the sport ethic dictates that athletes should remain optimistic that they can overcome any obstacle (e.g., equipment failure, rule changes, officiating bias). Athletes are expected to maintain this state of eternal optimism, regardless of any adversity, else the athlete faces a diminishing support system for abandoning The Game.

Although many of the previous examples demonstrate an exception to the rule, rather than the rule itself, the expectation remains the same. That is, overconforming behaviors are said to differ "in degree, not in kind" from those behaviors expected of all athletes in elite sport (Hughes \& Coakley, 1991, p. 316). Therefore, whereas a small percentage of athletes overconform compared to their normally-conforming counterparts, all athletes, regardless of their degree of conformity, are exposed to each of the norms of the sport ethic.

Originally, those athletes proposed to be most at risk for overconforming to sport norms are athletes who (1) have a low self-esteem, leading them to develop a strong need for acceptance by their athlete peers, and (2) believe that athletic success is the most reliable means to gain respect from their peers (Hughes \& Coakley, 1991). More recently, Coakley (2015) added that athletes at risk of overconforming also include those that combine measures of masculinity and athletic identity into a single, meaningful identity. Waldron, Lynn, and Krane 
(2011) echo this sentiment, clarifying that athletes with strong social approval goal orientations are driven to demonstrate their identification with hegemonic masculinity to gain social approval. Additionally, Coakley (2015) postulates that athletes in elite sport are vulnerable to the expectations of the sport ethic given that as they increase in competitive level, and thus training intensity and frequency, their support from outside sources who might question the legitimacy of strict adherence to the sport ethic is minimized. That is, athletes receive increased sport-specific support from coaches, athletic trainers and physicians, psychologists, and nutritionists, among others. The more they are required to train in the controlled sporting environment, the less able they are to take advantage of outside sources of support that might differ in opinion, advice, and alternative resources. Therefore, they are more often reinforced and encouraged to overconform, than their peers, in an effort to continue training with the desired group.

\section{Athletic Identity Development}

At its core, deviant overconformity was originally thought to be the standard by which athletes solidify their "athlete" identity (Hughes \& Coakley, 1991). Hughes and Coakley (1991) proposed that the process by which athletes adopt overconforming behaviors occurs through a series of identity construction and confirmation stages as explained by Donnelly and Young (1988). Included in the process are the stages of presocialization, selection and recruitment, socialization, and acceptance or ostracism. Throughout the process, the athlete moves from gathering information about a sport subculture (i.e., presocialization) from outsiders to that subculture to achieving membership within that subculture (i.e., selection and recruitment) to learning about the true nature of the subculture known only to its members (i.e., socialization) and ending with either acceptance or rejection from other members of the subculture (i.e., acceptance or ostracism). 
It is during the socialization stage that aspiring athletes must make the decision whether to conform to the norms expected for membership in the elite group of individuals who can call themselves athletes or reject those norms and lose their membership. However, it is in the final stage that athletes are sometimes required to demonstrate their conformity to the norms of the sport ethic, and condoned by the subculture, by engaging in behaviors previously considered negative. Regardless of the conflict between what athletes believed about whether certain behaviors were acceptable or unacceptable in the sport context during the presocialization and selection/recruitment stages, failure to demonstrate elicit acceptance in the socialization stage, results in ostracism from the group in the final stage. Therefore, if an athlete is unwilling to participate in the behaviors condoned by the subculture and perceived as integral to solidifying membership, the individual is rejected from the group and cannot establish his/her identity as an athlete (Donnelly \& Young, 1988).

\section{Social Approval Goal Orientation}

Whereas the athlete identity was proposed as the foundation of deviant overconformity in Hughes and Coakley's (1991) original work, they also noted that athletic participation, and acceptance as "athlete," leads to a coveted status among athlete peers. Therefore, more recent literature has emphasized the role that social motivation plays in deviant overconformity (Waldron \& Krane, 2005). Focus on the importance of the role of social motivation on sport participation is in reaction to the idea that other theories (e.g., Achievement Goal Theory; Duda \& Nicholls, 1992) emphasize the motivating effect of demonstrating physical competence as a primary goal of sport participation. Proponents of the social motivation approach, on the other hand, argue that the sense of belonging, itself, is a goal of sport participation, rather than a byproduct that supports the goal of demonstrating competence in the sport setting (Allen, 2003). 
Given that social hierarchies are developed on the basis of conforming to the sport ethic (Waldron, Lynn, \& Krane, 2011), “athletes' attempts to fit in with teammates results in deviant overconformity" (p. 113). This is not to say that other motivations (e.g., demonstrations of competence) are not important driving forces of participation in sport. However, the focus on social motivation, as well, broadens understanding of motivation in sport, which allows researchers and practitioners to better understand sport behavior.

Social approval goal orientation is one facet of the social motivation to engage in an activity. That is, social motivation drives participants to engage in particular activities in an effort to capitalize on opportunities to build social bonds and receive support and validation from others (Allen, 2003). By examining the important driving force of creating and maintaining social bonds in sport settings (i.e., social affiliation orientation), as well as the driving force that athletes' sense of selves is reinforced by receiving validation from others (i.e., social validation goal orientation), one avoids the oversimplification of sport participation on the basis on proving physical ability, alone. Social validation, specifically, is derived from social approval, acceptance, and status. Athletes who are oriented to these outcomes, then, are motivated to participate in sport given the social benefits it can supply. As a result, athletes with high social validation orientation experience feelings of success only when they have attained the outcomes of approval, acceptance, and status. The motivation to obtain approval, acceptance, and status, then has "an impact on their feelings and actions in sport" (p. 554). Presumably, such actions may include overconforming behaviors if those behaviors are believed to contribute to physical competence and social validation. Later work, by Waldron and colleagues (Waldron \& Kowalski, 2009; Waldron \& Krane, 2005; Waldron, Lynn, \& Krane, 2011) suggests that social approval goal orientation is, in fact, related to overconforming behaviors, including hazing, 
disordered eating and steroid use.

\section{Overconformity in the Research}

The process of identity development and social acceptance has been documented in relation to overconformity in a handful of studies. Namely, Curry (1993) illustrated, through photograph and interviews with one collegiate wrestler, how the socialization process serves to norm such behaviors as playing in pain and/or while injured as an acceptable part of sport participation and that athletes who do so become role models for future athletes. Throughout his work with one athlete, Curry (1993) provided an illustration of Nixon's (1991) content analysis of the ways in which pain is handled in sport ${ }^{1}$. In his published account of that work, Nixon (1993) produced a typology of the sociological forces in sport that lead to the hiding, disrespect, negative evaluation, and depersonalization of pain that then leads to longer-term beliefs that pain and injury undermine masculinity but overcoming them can prove one's masculinity and doing so builds character. Specific sociological influences that impact athletes' perceptions of pain and injury include: (a) structural role constraints (i.e., expectations that the athlete is disposable, that there is something wrong with injured athletes, that the body must be pushed to perform optimally), (b) structural inducements and support (i.e., the athlete role is enticing given the potential for status, belonging, adoration, and support), (c) cultural values (i.e., character, pain tolerance, proving masculinity), (d) institutional rationalization (i.e., injury is part of the game but should not interfere with it, medical personnel can and do minimize the effects of injury, athletes' bodies are to be used in service to the team), (e) socialization of athletes (i.e., how

\footnotetext{
${ }^{1}$ Nixon (1991) originally presented his framework for how athletes respond to pain and injury in sport and the cultural influences that affect those responses at a national conference. His original presentation was referenced in Curry's (1993) work, cited here from Curry. Nixon eventually published that work in 1993, which is also included. The reader should note, however, that Nixon (1991) and Nixon (1993) refer to the same work, published first as a presentation (1991) and cited by Curry (1993) and later as a manuscript, cited in the present study.
} 
athletes "learn and internalize expected and desired behavior, roles, beliefs and feelings and develop self-conceptions" (p. 187) such as demonstrations of commitment to the game, loving sport unconditionally, experiencing pride and sense of immortality, trusting others to take care of them), and (f) accepting the risk of pain (i.e., the willingness of athletes to play hurt, blame themselves for injury, and push the body, despite the risks). Although Hughes and Coakley's (1991) work was not cited by Nixon (1993), conceptually there is much overlap between the two. More recently, Schnell and colleagues (Schnell, Mayer, Diehl, Zipfel, \& Thiel, 2014) attempted to identify athletes more likely to take risks that often lead to injury using 1,138 young, elite German athletes. They found that that athletes who were highly involved in elite level sport prioritized sport above non-sport domains (i.e., sacrificing for the game; Hughes \& Coakley, 1991) and those who tend to be more perfectionistic and focused on winning (i.e., egooriented motivational climate; Duda \& Nicholls, 1992) were most likely to take risks that might lead to injury. Given that many coaches and athletes in the United States subscribe to the idea that more is better and that athletes should "act tough and give 110\%" (Weinberg \& Gould, 2015, p. 462) it makes sense, theoretically, that Schnell and colleagues' (2014) findings may translate to collegiate wrestlers in the United States. In fact, Vetter and Symonds (2010) found that, among 149 Division II collegiate athletes, 50\% had chronic injuries, $24-31 \%$ felt physically exhausted frequently during competition season, and, even in the off-season, $18-20 \%$ were frequently physically exhausting. The former finding may be indicative of the norm to play through pain and injury (Hughes \& Coakley, 1991) whereas the number of athletes who were physically exhausted in season may be more related to the drive to distinguish oneself from others (1991). The latter finding might also reflect the cultural norm of sacrificing for the game (i.e., by maintaining intense training in the off-season, one sacrifices recovery; 1991). However, 
given that the sport ethic was not explicitly examined in either Schnell and colleague's (2014) or Vetter and Symond's (2010) work, the previous suppositions are a matter of conjecture, requiring further research.

Studies that have explicitly examined the role of the sport ethic on athlete identity and/or overconforming behavior include: John's $(1993 ; 1998)$ qualitative work with rhythmic gymnasts' and distance runners' disordered eating behavior and attitudes and Atkinson's (2011) ethnography of the culture of disordered weight control in collegiate and elite male runners, rowers, wrestlers, swimmers, gymnasts, skiers, and tennis players; Pettersson, Ekstrom, and Berg's (2012) research on the mental advantage gained by elite combat sport athletes who demonstrate their commitment to the sport using strict weight regulation practices; Waldron and Krane's (2005) investigation of the role of the sport ethic and achievement goal orientation on excessive violence, disordered eating behaviors, and steroid use in female athletes; Waldron and colleagues' (Kowalski \& Waldron, 2010; Waldron \& Kowalski, 2009; Waldron, Lynn, \& Krane, 2011) qualitative work on hazing in sport as a function of overconformity and social approval motivation; Heil's (2012) examination of the pain experience during the injury process in a distance runner and Fenton and Pitter's (2010) investigation of the role of socialization to the sport ethic on injury experiences in male rugby players. In each, the take-away remains the same. Behaviors athletes engage in as a result of their unquestioned adherence to the sport norms are not considered deviant within the sport culture. Whether athletes recognize those behaviors as harmful or not, they consider engagement with them to be an expected outcome of sport participation. Thus, if they value their sport participation and want to continue their involvement, they engage in overconforming behaviors with just as much commitment and discipline as they show up for practice. 


\section{Disordered Eating}

Whereas a preoccupation with weight has permeated society as a whole, when that preoccupation is included as part of sport, as an expected preparation strategy that athletes maintain a low body weight to perform well, it can lead to obsession, and, eventually, subclinical eating disorders (Johns, 1998). Throughout his work with rhythmic gymnasts and endurance runners, Johns $(1993,1998)$ uncovered repeated examples of the institutional expectation that athletes maintain and low body weight and countless examples of athletes' adherence to that expectation. The enduring acceptance of and adherence to weight-related expectations in sport as a symbol of their commitment and success, despite negative health consequences, was demonstrated again and again by athletes who both bought into the sport ethic and those who knew their behaviors were unhealthy, yet chose to engage in them, regardless, so they could continue their participation in sport.

Beyond the importance of demonstrating commitment to the sport ethic through disordered eating and other unhealthy weight management practices, an enduring belief that engaging in weight regulation provides the athlete with physical and mental advantages is also present. In a qualitative analysis of combat-sport athletes, Pettersson and colleagues (2013) found that participants reported the importance of weight regulation not only as a measure of sport identity, but also of self-discipline, control, focus, preparation, confidence, and power. Participants in that study still widely endorsed weight-regulation practices for the physical advantage afforded, but also discussed how important weight-regulation, as a symbol of the athlete's identity, mental strength, and mental power, is to gain the mental edge over their competition.

Disordered eating has been a major area of focus in the sport of wrestling over the last 
two decades. Although much research has investigated weight-loss strategies in high school athletes, many fewer have looked at weight loss practices in collegiate athletes. One such study found that although no athletes in the sample, which included athletes from sixteen different sports, self-reported scores significant of a clinical eating disorder, $20 \%$ reported symptom severity and frequency to be considered symptomatic (Petrie, Greenleaf, Reel, \& Carter, 2008). Worrisome is the finding that exercise for weight loss was the primary form of pathogenic weight control behavior (37\%) followed by fasting/dieting (14\%). Moreover, approximately $10 \%$ reported engaging in vomiting, laxative use, and/or diuretic use.

These findings are important given the perceived culture of wrestling. Specifically, following a series of three wrestler deaths in 1997 related to weight cutting and disordered eating, the NCAA changed its regulations to prompt a healthier weight management climate (Gibbs, Pickerman, \& Sekiya, 2009). In those regulations, wrestlers are prohibited from competing below a weight consistent with $5 \%$ body fat (Loenneke, Wilson, Barnes, \& Pujol, 2011). However, some debate exists about the reliability of measures used to predict a wrestler's weight at $5 \%$ body fat, prompting the call for future research on validation measures used to determine whether wrestlers are allowed to compete at matches. In a follow-up study about the effectiveness of the rule change, $40.2 \%$ of participants from 43 NCAA wrestling teams indicated that the rule changes deterred extreme weight loss behaviors. Regardless, many weight loss behaviors still existed, especially among freshman and lower weight-class wrestlers, including: gradual dieting $(79.4 \%)$, increased exercise $(75.2 \%)$, fasting $(54.8 \%)$, sauna use $(27.6 \%)$, and rubber/plastic suit use (26.7\%; Opplinger, Steen, \& Schott, 2003). Results indicated that those significant others most influential on decisions regarding weight management were coaches and peer-athletes. Of note, the use of saunuas and rubber/plastic suits are methods prohibited by the 
NCAA's new rules (Gibbs, Pickerman, \& Sekiya, 2009). Therefore, although the rule changes have been an effective deterrent in some respects, extreme weight loss practices still occur and are thought to be the result of long-standing mindsets about weight and performance held by athletes, coaches and clinicians (2009).

\section{Hazing}

As a recent national study on collegiate students indicated that varsity athletes experienced, on average, the highest rate of hazing encounters compared to every other social/activity group on campuses (Allen \& Maddsen, 2008). Explanations for the willingness to participate in hazing as a perpetrator, complicit party, or victim have been offered in recent years. The work of Waldron and colleagues (Waldron \& Kowalski, 2009; Waldron, Lynn, \& Krane, 2011) indicates that deviant overconformity may be one explanation for the many athletes who have been and continue to be involved in hazing practices. Specifically, their qualitative analyses suggested that the combination of the beliefs that being a part of the team required sacrifice and the endurance of the pain demonstrated commitment to the team, were at the heart of their participants' involvement in hazing behaviors. They also suggested that the desire to be part of the team, to be accepted by their peers, was a strong driving force. Those who conformed to the hazing behavior were accepted as members of the team, whereas those who did not were ostracized from the team. Moreover, in a study that examined athletes' perceptions of coaches' involvement, Kowalski and Waldron (2010) found that although some athletes perceive that their coaches do not accept hazing practices on their teams, others do. Levels of acceptance ranged from ignoring hazing all together to attempts to control it, to encouraging it. Thus, if uncritical acceptance to the sport ethic is the standard by which team membership occurs, hazing is likely to continue to be a problem on athletic teams. 


\section{Excessive Violence}

The idea that athletes view their bodies as machines drives much of the insight into why athletes may intentionally harm their opponents during competition (Waldron \& Krane, 2005). Specifically, when athletes perceive their bodies as machines, to be sacrificed and endure pain and injury, where the potential for greatness is considered limited only by lack of effort and commitment, athletes are more likely to put their bodies, and those of their opponents, on the line. This perspective is thought to be tied to masculine norms that demonstrate power and performance, thus requiring demonstrations of physical superiority.

\section{Pain/Injury}

Because athletes perceive their bodies as machines, tools to be used to secure success, they may minimize, disrespect, negatively value, and depersonalize pain (Curry, 1993). According to Waldron and Krane (2005), "When bodies are considered machines, athletes' minds and bodies become disconnected. While the mind focuses on winning at any cost, the body can be compromised for the good of the cause. Thus, "real' athletes endure pain and take risks" (p. 320). Young (1993) analyzed the risk of injury in sport from a workplace standpoint, suggesting that the legal notion of violenti, or the assumption that one willingly accepts the risk of injury in the workplace, explains much about how athletes view pain and injury in sport. Similarly, in a study of Canadian male athletes (Young, White \& McTeer, 1994), the risk of serious bodily injury was minimized given the belief that participation in violent sport and the willingness to take the risk of serious injury are considered displays of masculinity. Fenton and Pitter (2010) added to the understanding of pain minimization in rugby players, also including that if an athlete succumbs to the pain or an injury and leaves the field, he is not permitted to return. Therefore, if athletes acknowledge the pain, they are no longer allowed to keep playing 
for the remainder of the match. This regulation in rugby, then, has led many ruggers to increase their pain tolerance and mask signs of injury from their coaches and staff using methods they have learned from modelling and previous experience.

\section{Performance Enhancing Substance Use}

The use of anabolic steroids, erythropoietin (EPO) and techniques like blood doping to improve performance have been the subject of media and research attention in recent years due to the dangers of taking substances not regulated by the Food and Drug Administration (FDA) and prohibited by governing bodies like the World Anti-Doping Agency (WADA) which diminishes close medical supervision (Coakley, 2015). Although better understanding and regulation of the use of these performance enhancing substances is important, stakeholders in sport may have overlooked the dangers of the use and misuse of painkillers in sport for pain management, further blurring the line between substance use for necessary medical treatment and substance use for performance enchancement. Specifically, Cottler and colleagues (2011) found that $52 \%$ of the professional football players surveyed used opioids during their careers to manage pain, with $71 \%$ reporting that they misused opioids and $63 \%$ obtained them through illegal practices at some point in their careers. They found that the prevalence rate of opioid use was $7 \%$, which is three times the prevalence rate of use in the general population. Although the long-term effects of misuse in the sport setting are not well-understood given confounding variables like undiagnosed concussions and heavy alcohol consumption, misusing opioids leads to an increase of future misuse and abuse. Just as institutional pressure to use anabolic steroids, EPO, and techniques like blood doping influence athlete choices to use, institutional pressures to take painkillers also affects athlete choices to use (King, et al., 2014). Analysis of the cultural forces that influence choices to use included identity development, race, gender and labor. 
Implicit in those forces is the role of masculine norms, which are embedded in the wider culture of sport, and voluntary assumption of risk (i.e., violenti, Young, 1993) on athletes' use and misuse of painkillers to maintain athlete identity and continue playing sport. Perhaps a distinction is made between the use of steroids as a form of creating unfair advantage, which demonizes users (i.e., rate-busting, Heckert \& Heckert, 2002), and the use of painkillers as a sign of an athlete's commitment to sacrifice, accept no limits, and strive for distinction, labeling users as heroic (i.e., positive deviance, Heckert \& Heckert, 2002; King et al., 2014). Regardless, the side effects of both types of drugs is cause for concern in the sport subculture. 
APPENDIX B 


\section{Extended Methods}

What follows is an in-depth exploration of the researcher's worldview, as a researcher, as well as of the selected methodologies. In order to better understand the researcher's choice in methodology, it is important to understand how it fits with her general views on how and what types of information can be known. From there, it becomes easier to understand the chosen research design and associated methods within the context of the current study. Consistent with the researcher's worldview, she will use first-person language when discussing her research philosophy.

\section{Researcher Philosophy of Science}

When compiling a research plan, explicitly stating the philosophical worldview, or paradigm, assists the reader in understanding the rationale for the chosen approach (Creswell, 2009). To accomplish this task, it is necessary to address the researcher's worldview or "basic set of beliefs that guide action" (Creswell, 2007, p. 19). These beliefs address a number of areas surrounding the nature of knowing, including ontology, epistemology, axiology, rhetoric, and methodology, as well as the overarching paradigm that encompasses those beliefs.

\section{Ontology}

At the basic level, ontology is the nature of reality and its characteristics (Creswell, 2007). Within this philosophy, there are two ideas about the nature of reality: (a) that there is one, universal truth that is the only reality and, (b) that reality is created from multiple truths that are grounded in individual perception (Ponterotto, 2005). These ideas can then be further broken down into truths that can be uncovered and defined, and those that cannot. For instance, some researchers (i.e., positivists) believe that there is one single truth that can be discovered, explained, and predicted; others (i.e., post-positivists), however, believe that one true reality is 
out there, but that it cannot be identified.

According to a subjectivist or relativist ontology, there are multiple realities, realities that are constructed by individual experiences. Therefore, "there are as many realities as there are participants (plus one: the investigator)" (Morrow, 2007, p. 213) and the meanings ascribed to those realities are a result of the interaction between researcher and participant, in addition to all previous experiences. Lauer (1967) once said, “[o]nly one source of certainty exists, what I think, what I feel, in substance, what I perceive" (cited from Moustakas, 1994, p. 26). Of note here is that reality, according to an ontonology of multiple truths (i.e., subjectivity), is not only constructed by the current experience, but also by past experiences and social context (Corbin \& Strauss, 2008).

A third, hybrid ontology in which researchers believe that an external reality exists, but that it is only known through individual lenses of perception has been termed "constructionist" (Crotty, 1998). Admittedly, this is the core ontology I most relate to. Although the subjective side of the constructionist ontology is emphasized in my current research study, the objectivist side of me occasionally influenced my interpretation.

\section{Epistemology}

Epistemology, on the other hand, is the study and acquisition of knowledge. In essence, whereas ontology is concerned with what the reality is, epistemology is concerned with how that reality is known by fixtures within that reality. It is concerned with the relationship between the knower and the would-be knower; namely for the purposes of research, the relationship between the participant and the researcher. Traditional epistemologies dictate that the researcher either maintains an objective ideal with her participants or takes a more subjective stance (Ponterotto, 2005). In accordance with qualitative research, I took a more subjective stance in my current 
work in an attempt to minimize the distance between me and my participants (Creswell, 2007). Due to the sometimes negative connotation of "subjectivity" in scientific research, I refer to sensitivity, as proposed by Corbin \& Strauss (2008) instead. In this way, I immersed myself in the lives of each participant in an effort to better understand the context of that participant.

As Dewey (1938) said, "Neither inquiry nor the most abstractly formal set of symbols can escape from the cultural matrix in which they live, move, and have their being" (p. 20; cited from Corbin \& Strauss, 2008). According to pragmatists, understanding these meanings is a function of social and cultural context that cannot be separated from each other (Corbin \& Strauss, 2008). Further, Dey (1993) explained that "there is a difference between an open mind and an empty head. To analyze data researchers draw upon accumulated knowledge. They don't dispense with it. The issue is not whether to use existing knowledge but how" (p. 63; cited from Corbin \& Strauss, 2008). If I strive to separate my interpretation of the data from my own previous experiences, I am separating my understanding of meaning from my cultural and social context — a feat deemed impossible by pragmatists like Dewey. Therefore, it was my goal to be sensitive to understanding how my past experiences and perspective of reality influenced my interpretation of my participants' reality and how I came to interpret their realities that way. As Corbin and Straus (2008) clarify, "our findings are a product of the data plus what the researcher brings to the analysis" (p. 33).

\section{Axiology}

A third consideration while conducting research is the role and place of values in the research process, also known as axiology (Ponterotto, 2005). As with ontology and epistemology, two major dichotomies of axiology exist: value neutrality and value awareness. In traditional quantitative research, the researcher strives to neutralize values, attempting to collect 
and analyze data without the influence of researcher values (Creswell, 2007). However, this process is often difficult, especially in qualitative research, where the researcher is in greater contact with the participants. Additionally, the researcher sometimes investigates phenomena with which she has had experience. As experiences shape individual realities (Corbin \& Strauss, 2008), it is inevitable that the researcher develops values and biases based on those experiences.

Therefore, I strove for value awareness in the current work. I understood that my values and biases would invariably color my interactions with participants and my interpretation of the data. Instead of ignoring my values and biases, hoping to operate in their absence, I sought to “position” myself (Creswell, 2007, p. 18) in the research by understanding my own values and biases and evaluating how those impacted my research. To accomplish this task, a number of self-reflexive strategies were employed; see Researcher as Instrument for more information.

\section{Rhetoric}

In the reporting of research, two major types of language, or rhetoric, are present. The first is a more scientific approach, striving to depersonalize the researcher. This rhetoric is common in the positivist and postpositivist schools of thought (Ponterotto, 2005). A first-person language, however, is often used for researchers in constructivist and criticalist paradigms. The second option details the expectations, values, and biases of the researcher, paying special attention to the impact on the research process and emotional and intellectual evolution of the researcher as a result of the project. Further, Caulley (2008) suggests that striking a balance between first- and third-person language is appropriate. Specifically, he suggests using firstperson language in the methods sections of a manuscript, given that the researcher can speak to first-person experiences. For the remainder of the document, he recommends speaking in the third person, given that, however intimately the researcher is aquainted with past research and 
participant data, she can never truly know the experience. The current study sought to reflect Caulley’s (2008) axiology.

\section{Methodology}

The methodology of research addresses the process and procedures of that research (Ponterotto, 2005). Methods of research can be either deductive or inductive in nature. Methods that are deductive begin with a theory or question and form an a priori hypothesis. Specifically, the researcher has an idea of what the explanation for the phenomenon is and then seeks to confirm that hypothesis throughout the research process. Conversely, an inductive methodology seeks to uncover meaning as the research progresses. The researcher explores a phenomenon from the ground up, allowing the description and meaning of the phenomenon to emerge and change based on the researcher's experiences during data collection and analysis. Given the nature of inductive methodology, even the research question may change throughout the process, evolving as more information is obtained and new meanings emerge (Creswell, 2007). It is a balance of both methods that drove the current study. Although I had ideas about how the sport subculture affects identity formation, and the relationship between identity development and deviant overconformity, the mechanisms by which those changes occur is largely unknown and a change in the research question may have been warranted.

\section{Paradigm}

As stated earlier, the researcher's philosophy of science (i.e., the combination of ontology, epistemology, axiology, rhetoric, and methodology) lends itself to specific philosophical worldviews or paradigms (Ponterotto, 2005). The current study was nested within the constructivist-interpretivist paradigm. Kant once said that "human perception derives not only from evidence of the senses but also from the mental apparatus that serves to organize the 
incoming sense impressions" (cited from Ponterotto, 2005). Specifically, the meaning of a phenomenon is constructed as individuals engage with the environment (e.g., Corbin \& Strauss, 2008; Creswell, 2009).

Within the field of sport psychology, students are often reminded that perception is reality; we are told that it is important to remember that although the actual occurrences influencing athletes may seem one way to the consultant, the important thing is how the athlete perceives those occurrences. It will not matter what "true" reality is to the athlete struggling with performance anxiety or injury recovery, he or she will appraise the situation based on his or her perceptions of that reality in concert with previous social interactions and personal history. As this view is personal to the individual athlete, understanding his individual reality takes some research in itself. The consultant must work with the athlete, asking questions and encouraging reflection to understand the "hidden" reality the athlete perceives and make sense of his experience. For the current study, given my belief that perceptions reflect reality and that the meaning of that reality is complex, requiring critical investigation, the constructivistinterpretivist paradigm was appropriate.

Additionally, I subscribe to the idea that the mere act of conversing with the athlete and investigating how life influences the participant's reality—as it is yet another experience that works to shape the athlete's reality_alters that athlete's present perception of reality. If, as the relativist ontology of multiple realities suggests, athletes perceive reality based on their own experiences, social interactions, and personal history, the social interaction of an athlete with a researcher will influence his perceptions of reality, both in the recollection of events and in the appraisal of future events. This second philosophy speaks to the importance of understanding the context of the athlete, a practice that is encouraged within the constructivist-interpretivist 
paradigm.

"[M]ethodologies may firmly rule out personal experience from inquiry in the name of 'objectivity.' Then, too, they may undervalue... the importance of self-reflection both in its relation to what reality 'is' and to its role in 'knowing' it”' (Strauss \& Corbin, 2008, p. 5). Researchers are not exempt from forming their own perceived reality based on past experiences, social interactions, and personal histories. As such, it was not unlikely that I carried various values, beliefs, and biases surrounding that reality. Therefore, it would be naïve to believe that I could interpret participant reality without some influence of these values, beliefs, and biases. My subsequent recognition that my interpretation of the athlete's reports would be filtered through my values and biases is encouraged within the constructivist-interpretivist worldview.

Finally, the current study seeks to understand how wrestlers define the athlete identity and experience deviant overconformity. It was framed within a constructivist-interpretivist paradigm. As a result, I sought to identify participant identity and overconforming behaviors inductively and deductively, providing the research community with a better understanding of how wrestlers define the athlete identity and their experiences with deviant overconformity.

\section{Qualitative Research Strategy}

For the current study, a combination of life story interview and semi-structured interview was chosen to investigate the meaning attributed to the athlete identity, as defined and experienced by wrestlers, themselves. By combining life story and semi-structured interviews, I was able to get a picture of the interaction between participants' personal experiences with the athlete identity, including its roots and consequences, and the sociological culture in which the athletes lived, including its roots and consequences. Therefore, a greater understanding of the narrative methodology is first presented, followed by a summary of life story interview methods 
and semi-structured interview methods. Then, a discussion about my proposed role was presented in depth to supplement previously presented information and clarify how and why I designed my study. Finally, a detailed description of the data analysis is offered.

\section{Narrative}

"A difference between narrative analysis and either everyday or performance storytelling is that analysts ask how people's lives are defined as much by the stories they overlook as by those they do become caught up in" (Frank, 2010, p. 4). Narrative methodologies share the foundation that story is central to the self; that without stories, one would lack understanding of who one currently is as well as who one might become (Marshall \& Rossman, 2006). Narrative designs attend to “diversity, contradictions and failures of meaning, research participants' own generations of meaning, and to the mutual constitution of meanings between participants, researchers, the research context and the wider context" (Esin, Fathi, \& Squire, 2013, p. 203). That is, they are seen as appropriate vehicles for understanding identity (Kraus, 2007), which is at the heart of the current study.

Narrative research has been recently argued for in the domain of sport and exercise psychology for a variety of reasons (Smith, 2010). For one, some researchers have reacted negatively to post-positivist methodologies that, some feel, lack the depth of context inherent in the athlete experience. For others, questions of the ability of the researcher to adequately communicate the athlete experience arose. Consequently, several researchers have turned to an interpretivist paradigm which supports the study of context and individual interpretation as the cornerstone of understanding reality (Carless \& Douglass, 2013a, b; Smith \& Sparkes, 2009a). Other researchers (e.g., Vajda, 2007), however, have turned to narrative research given its potential applied and analytical benefits (e.g., therapeutic benefits of the act of storytelling, 
richness of data collected and analyzed) and opportunities for creativity in research. Moreover, as a "social artefact," stories tell about the individual and the society, simultaneously. This last point allows the researcher to understand the person, the environment, and the complex interaction between the two instead of attempting to control them. Furthermore, in sport and exercise psychology, particularly, many phenomena are studied from an interactional perspective, reflecting the concept of reciprocal determinism which states that just as the individual is affected by the environment, he also affects it (Weinberg \& Gould, 2015). The ability of narrative designs to address these issues makes it appropriate in the sport and exercise setting.

Although various definitions abound, narrative may be defined as "a complex genre that routinely contains a point and characters along with a plot connecting events that unfold sequentially over time and in space to provide an overarching explanation or consequence" (Smith \& Sparkes, 2009a, p. 2). The goal of narrative research is to interpret the perceptions, meaning making, and social actions of participants, focusing on the how and what of the story at the same time (Smith, 2005; Smith \& Sparkes, 2009b). Further, an important distinction has been made between stories, which are the product of narrative, and narrative, which is the process and structure of telling stories. Regardless, the two are necessarily linked. As humans, we "live in, through and out of narratives. They serve as an essential source of psycho-socio-cultural learning and shape who we are and who we might be" (Smith \& Sparkes, 2009a, p. 3). "Stories always pose that question: what kind of truth is being told? Stories never resolve that question; their work is to remind us that we have to live with complicated truths" (Frank, 2010, p. 5). Stories guide how we see the world and the things we do in that world. The human experience involves "lead[ing] storied lives" (Smith, 2010, p. 87). 
As such, narratives create meaning for, adapt existing meaning of, and receive meaning from the human experience, which makes them essential to understanding the self (McAdams, 2001). Moreover, the power of the narrative is that it exists only in the presence of real or imagined others, thus it is both personal and social (Smith \& Sparkes, 2009a). To add further complexity to the function of narratives, because they are personal, as well as social, the narrator exercises some control over what stories are told to whom, in what ways at what time, and with what details, within the context of cultural narratives. In part, the narrator's choices are made with reference to the cultural narratives available at the time, creating a template for the construction and performance of the story, and in part from the narrator's fluid and dynamic sense of self (Smith, 2010). The result of which, in addition to personal meaning that fulfills the individual experience, is that narratives are co-created by the narrator and the audience, shaping the social identity afforded to the narrator. Because people are socialized to ascribe certain cultural meanings to cultural experiences, which are understood through the individual lived experience, the narrative audience (i.e., real or imagined others) in part determines the meaning of the experience. Therefore, as sense cannot be made of non-sensical stories, the story told must be convincing to the audience of real or imagined others in order to carry any meaning to the individual, thus shaping and guiding that individual's identity (McAdams, 1998) as well as future behavior within the culture (Smith \& Sparkes, 1998). From this perspective, when doing narrative research, it is important to keep in mind that the researcher becomes the audience for the particular story shared. Accordingly, because narratives are interactive in this sense, the researcher becomes a co-author in the creation of the narrative under study (Smith \& Sparkes, 2009a).

\section{Life Story Research}


Life stories, a specific type of narrative inquiry, assist the individual with selfunderstanding in a cultural context. That is, individuals make sense of themselves and their social worlds through a process of unifying their experiences into a coherent explanation of who they were, are, and wish to be. According to McAdams' (1985) Life Story Model, "identity is an internalized life story" (2001, p. 101).

Marshall and Rossman (2006) outline five criteria for life histories: (1) the individual is accepted as a member of the culture, (2) the data should include the role played by significant others in conveying socially-constructed realities (3) the data should include the rules, codes, rituals, and myths of the culture, (4) the data should focus on capturing the process of the phenomenon for the individual, and (5) the culture should be understood through the lens of the individual's life story. To address the first criteria in the current study, participants were chosen on the basis of their membership in the collegiate wrestling subculture. The life story interview guide for the current study was adapted from McAdams and Guo's (2014) work with the remaining criteria in mind.

Life stories tend to focus on "critical or fateful moments" that help make sense of the socialization process (Peacock \& Holland, 1993, p. 116). Additionally, life stories are "uniquely suited to depicting and making theoretical sense of the socialization of a person into a cultural milieu" (Dollard, 1935, as cited in Marshall \& Rossman, 2006, p. 115). Salient to this socialization process is an emphasis on understanding how individuals learn to meet the "normative expectations" of a culture or society (p. 116). Given that athletics, especially the subculture of collegiate athletics, is a largely closed society and that the norms and resulting behaviors I sought to explore lend themselves to secrecy except from group members, understanding its processes from the inside was invaluable. Additionally, this approach was 
particularly aligned with my epistemological stance in that "[life history researchers] assume a complex interaction between the individual's understanding of his or her world and that world itself” (115).

Although life stories are well-suited to the researcher's intent of seeking to investigate meaning, socialization and normative expectations, they may also be particularly beneficial to the participant. Given the relationship between life stories and individual identity (McAdams, 2001), participants of life story research may experience profound therapeutic benefits simply by engaging in the research process (Vajda, 2007). Regardless of the benefits of narrative research in general and life story research specifically, as with all methodologies, several critiques are worth noting. For one, concern over the believability of life stories has been voiced. Critics who share this concern typically site the inability for a story to be proven (e.g., Fogelson, 1979, as cited in Peacock \& Holland, 1993). Given that stories are co-authored by the narrator and the audience, and that the narrator holds the ability to choose what details to share about what stories that are told, believability of the story, in the absolute sense, is a valid argument. However, life story researchers, as interpretivists, are more interested in the process of meaning-making and the interpretation of the human experience, as opposed to the discovery of an absolute truth prized by positivists. Inherently interpretivist in nature, life story research seeks to understand the multiple realities created by the multiple interpretations of the lived experience of the individual experiencing life (Sparkes \& Smith, 2009). Thus, by shifting the focus from the absolute truth, an object solely outside the individual, to be captured and studied to the truth as perceived by the individual, the absolute believability of life stories is a moot point—whether an event did or did not occur in external reality is irrelevant when seeking to understand how the individual makes meaning of the world and choices about how experiences are shared with that 
world.

Another critique of life story research is that, if all stories are unique, attempting to study shared phenomena through the exploration of individual life stories is counter-productive, if not impossible (Peacock \& Holland, 1993). However, as McAdams (1998) notes, a unique life story and commonalities between life stories are not mutually exclusive. That is, while specific experiences may differ, they may share common elements worthy of study within the unique life story framework. Because life stories are co-authored by their audience, they reflect cultural values and norms, providing insight into cultural phenomena. As such, "they have two sides, one personal and the other social" (Frank, 1995, as cited in Smith \& Sparkes, 2009b). By learning about individual socialization to cultural norms, cultural norms are necessarily highlighted and understood in a context greater than the individual. Therefore, life story methodologies capitalize on the complex interaction of the social and the personal, seeking to better understand the socialization process by making meaning of individual identity formation within the cultural context in which it was created (Marshall \& Rossman, 2006). In keeping with the advantage of simultaneously studying the person and the culture, life stories have a "place as simultaneous creators and creatures of social, cultural, and psychological dynamics" (Peacock \& Holland, 1993, p. 374).

Once the decision was made to employ life story strategies, the researcher focused on how to do so. For college-aged participants, McAdams and Guo (2014) propose that a structured approach may facilitate the formation of identity by making explicit the process of identity exploration using narrative. For instance, by explicitly asking a participant to tell the story of the most challenging moment of his life, he is forced to make a choice about what story to tell. That choice, then, tells him something about how he understands himself, which shapes his identity. 
Had he made a different choice, he would likely have provided a different meaning of his experience, eliciting a different interpretation of his identity.

\section{Role of the Researcher}

I tend to lean towards a social constructionist epistemological stance (Crotty, 1998), with strong interpretivist curiosities. I agree that objects exist, with or without human consciousness, but that the meaning ascribed those objects is integral to the understanding of our world. This meaning is created through two processes: the first of which is from the meaning we are socialized to understand as it was created by the greater collective culture (i.e., larger societal cultures, as well as smaller subcultures); the second is through individual experience and interaction both with that culture and with the object, itself. In the present case, the meaning of "athlete" and "sport" is created by the greater collective culture in North America, as well as by the subcultures of the specific sport, region, level of competition, and team. They are also created by the athlete within that culture, as a product of his experiences in North America, the specific sport, region, level of competition, and within the specific team.

Moreover, if the individual athlete is shaped by each interaction between himself and the individuals within various cultures of which he is a part, he must also be influenced by his daily interactions not directly related to sport. Therefore, I tend to believe that the researcher is hardpressed to remove herself entirely from the research process. That is, I believe, as do others (Smith \& Sparkes, 2009a) that the interaction of the researcher and the participant, in part, shapes the story told by the participant, as well as the future of the participant.

Therefore, in qualitative research, it is necessary that the researcher makes known her values, biases, and experiences in relation to the present research. According to Creswell (2007), all "writing is 'positioned' within a stance” (p. 179). In an effort to position myself as a 
researcher within the research process, I strive to explicitly state my values, biases, and experiences as the existence of those values, biases, and experiences will influence my interactions with my participants, the stories they share (Smith, 2010; Smith \& Sparkes, 2009a), and my interpretation of the data (Creswell, 2009).

Stance on Subjectivity. "In the absence of an articulated perspective on subjectivity, researchers leave themselves open to questions regarding whose perceptions are really being described in the findings" (Morrow, 2005, p. 254). Although "bracketing" or becoming aware of biases and striving to set those aside during analysis is common in phenomenological research (2005), in keeping with narrative research traditions, the current study will be nested in a view of subjectivity that acknowledges biases, but instead of setting them aside, strives to acknowledge their impact throughout project conceptualization, analysis, and interpretation.

Methods of Reflexivity. In accordance with my social contstructionist ontology, and constructivist-interpretivist worldview, I set out to make my implicit values and biases explicit by exploring my experiences related to being an athlete and a coach at the collegiate level (see Appendix F for a background of the researcher's sport experience). To accomplish this task, I am taking part in a process known as reflexivity, or self-awareness. The goal of reflexivity is to cultivate the self-awareness and deal with assumptions created from personal experiences or during interactions with participants.

This process included methods such as analytic memos (see Appendix I; Saldana, 2013), the use of a peer research team and member checks (Morrow, 2005). By asking participants to complete two brief written exercises, the researcher acquires additional data to include in and provide context for analysis. The AIMS, SMOSS, and written expression exercise were analyzed following the second round of coding and used as methods of data triangulation. As a result, 
information gathered in written form served to add context to the analysis (e.g., level of participant identification as an athlete served to provide additional information about the athletic identity for each participant) and to better understand areas of discrepancy (e.g., using principles of the FCE, written expression about participants' observations of overconforming behaviors in other athletes may reflect a different level of participant overconformity than presented during the interview).

A researcher background statement served as an exercise in self-reflexivity (see Appendix F). In the researcher background statement, the researcher reflected on her experience with the athlete identity and overconformity in sport. By re-visiting her statement periodically, the researcher can analyze areas of bias and how they influence her interpretation of the dataset.

Analytic memos (Saldana, 2013) serve to document self-understanding and thought processes throughout the research process. It is recommended that analytic memos begin at the time the research process begins, and continue until the project is closed. The use of analytic memos to establish trustworthiness will serve two purposes. First, it will be one measure of selfreflexivity for the researcher, highlighting researcher bias during the data collection and analysis processes, allowing her to express the extent to which her biases affect her interpretation of the data. By examining her analytic memos periodically, she can increase her awareness of past instances of how her bias(es) influenced interpretations as well as monitor current and anticipated influences. Second, analytic memos will provide the researcher with a means to use research team members to check researcher bias without burdening them with full interview transcripts, AIMS and SMOSS results, and written expressions.

In order to maintain a balanced view of data collection and analysis, the use of a peer research team is warranted as the team may serve to reflect or refute the researcher's 
interpretations (Morrow, 2005). Following the completion of pilot data collection, the researcher asked two other researchers to assist with round two coding of her data. One member of the research team possessed extensive experience as an athlete and coach in another sport and familiarity with qualitative data analysis. The second member had expertise in life story research design in a different field and considerable experience as a national athlete. The research team met on three occasions to discuss participant selection, data coding, refine provisional codes, and draw the researcher's attention to potential areas of bias during interpretation (see associated analytic memos, Appendix I).

To ensure accurate representation of participant experiences, I will employ member checks, during which I will present interpreted data to the participants and check for accuracy (Morrow, 2005). To do so, I will invite participants to review their word clouds, as they are a much briefer form of data presentation than full interview transcripts or lengthy narrative constructions; however, both will be made available to participants, should they prefer to read them and provide feedback. When presented with his word cloud, the pilot participant expressed appreciation for the ease and brevity with which he could review his extensive data. He did not indicate any need for revisions, deletions, or additions.

Participantness, Revealedness, and Intensiveness/Extensiveness. Some final considerations regarding the role of the researcher should be made: participantness, revealedness, intensiveness and extensiveness (Marshall \& Rossman, 2006). Participantness refers to the degree to which the researcher is involved in the participants' life (Marshall \& Rossman, 2006). Researchers fall on a continuum from full participation, in which the researcher is completely immersed in the participants' daily activities, to complete observer, in which the researcher is not at all involved in the participants' daily life. For the current study, the researcher plans to take 
more of an observer role, limiting contact with participants to the interview/written exercise processes and member check processes, only. In this way, my biases will likely have less impact on the participants' perceived present world than daily observation would.

One ethical consideration in any type of research is the amount of disclosure the researcher provides the participants. Known as "revealedness," the researcher will fully disclose the purpose of the study, completely deceive the participant during data collection, or will fall somewhere in between (Marshall \& Rossman, 2006). The current study takes direction from Taylor and Borgdan (1984) who propose the researcher be "truthful yet vague" when explaining the purpose of the study (as cited in Marshall \& Rossman, 2006, p. 73). In this way, the researcher will avoid some social desirability responding without unduly deceiving the participant.

A final consideration for the role of the researcher is intensiveness/extensiveness. Intensiveness/extensiveness refers to "the amount of time spent daily in the setting and the duration of the study" (Marshall \& Rossman, 2006, p. 73). For the current study, the amount of time spent daily in the setting will be minimal, limited to two total meetings per participant. The duration of the study will be limited to a single athletic season.

Finally, trustworthiness is established on the criteria of width, coherence, insightfulness, and parsimony (Lublich \& Tuval, 1988). Specifically, width refers to the degree to which complete data has been sought out and collected. In order to demonstrate sufficient width, researchers are encouraged to include illustrative quotes from various data sources and suggest alternative interpretations so as to allow the reader to judge, for herself, the soundness of the researcher's findings. Coherence, then, refers to the degree to which the researcher presented a meaningful interpretation and analysis of the data as a single product. Coherence is judged on the 
basis that, internally, each portion of analyzed data fits with others, as well as externally, the overall picture fits within existing theories and research. Insightfulness refers to the originality of the research, such that it contributes to the reader's understanding of herself and the larger culture. Finally, parsimony is best evaluated to the extent that the results are presented concisely and elegantly. Using width, coherence, insightfulness, and parsimony as a measure of qualitative research quality has been endorsed by researchers in sport and exercise psychology who performed narrative, life story research (e.g., Carless \& Douglas, 2013a). Given the similarity of aims between that work and those of the current study, the evaluation of research(er) quality on the basis of width, coherence, insightfulness, and parsimony was deemed appropriate.

\section{Data Analysis}

One strength of narrative inquiry is that the content of the story (i.e., the what) and the process by which the story is told (i.e., the how) are presented simultaneously (Smith \& Sparkes, 2009b). Through analysis, the focus is on stories where individual meaning making and coauthoring are central as opposed to treating the data as reports where universal truths are sought to be uncovered. Moreover, the selected analyses allow for both the personal and the social to be examined together. As the chosen analyses are explained in detail, it is important for the reader to remember that " $[\mathrm{t}]$ here are no formulae or recipes for the 'best' way to analyse the stories we elicit and collect. Indeed, one of the strengths of thinking about our data as narrative is that this opens up the possibilities for a variety of analytic strategies" (Coffey \& Atkinson, 1996, p. 80 as cited in Smith \& Sparkes, 2009b, p. 281).

Following immersion in the data (i.e., iterative reading and re-reading transcripts in whole and in detail), data from the current study were analyzed in two stages: categorical-content and holistic-content (Lublich, Tuval-Mashiach, \& Zilber, 1998). In the first, the content of the 
narrative (i.e., the what) was analyzed using provisional coding. Provisional coding is performed by generating a list of approximately 12 to 60 codes prior to data collection. The list is compiled using information from previous literature, existing theoretical frameworks, research questions, prior researcher experience, and pilot data (Saldana, 2013). That list is then compressed, adapted, or expanded as data is collected, coded, and analyzed, resulting in many fewer major themes. Provisional coding is considered appropriate when studies build from previous research and theory, as does the current study. The specific provisional code list for the current study (see Appendix E) was generated using concepts from existing theoretical frameworks (see discussion of deviant overconformity [Coakley, 2009; Coakley, 2015; Hughes \& Coakley, 1991] and the construction and confirmation of identity [Donnelly \& Young, 1988]), existing research on overconforming behaviors (e.g., Coakley, 2009; Hughes \& Coakley, 1991; Waldron \& Kowalski, 2009; Waldron \& Krane, 2005), and information gathered from the pilot interview. Although analyzing with a starting set of codes makes comparison to theoretical frameworks and previous research an easier task, it can also lead the researcher to attempt to fit the data to the codes (Saldana, 2013). Therefore, it was important for the researcher to continually reflect on the role her personal biases (see Research Background, Appendix F), and researcher biases, generated from her knowledge of existing literature and frameworks, had on her data analysis. Using analytic memos (Saldana, 2013) helped her maintain self-awareness to avoid the trap of fitting her data to existing work and allow her to gain perspective about what the data actually presented (see Appendix I for selected analytic memos). Additionally, periodically sharing her coding and analytic memos with research assistants and committee members held her accountable to being open to the stories her data told.

In the second round of analysis, narrative analysis (Sparkes, 2005) was used to analyze 
the process by which the story was told (i.e., the how), to better understand how the greater sport culture affected the stories individuals told and their responses to cultural pressures. Narrative coding is considered appropriate for research lines investigating identity development and "psychological, social, and cultural meanings and values" (Saldana, 2013, p. 132). By examining the participant's story holistically, the researcher gained insight into the psychological, social, and cultural forces that comprise "the human condition" (p. 132). "The ultimate goal is to create a stand-alone story as research representation that may depict 'how and why a particular outcome came about' (Polkinghorne, 1995, p. 19)" (p. 134). The stand-alone stories for the current study followed Caulley's (2008) techniques for writing creative nonfiction.

Data from the current study was presented in two ways. In order to address the first research question, the researcher utilizes a word cloud program (Wordle; Feinberg, 2013) to illustrate how the participants defines the "athlete" identity. Word clouds are visual depictions of word/concept frequencies (McNaught \& Lam, 2010). Specifically, words that appear more frequently are afforded larger font whereas words that are rarely recorded are given a smaller font. Doing so allows the reader to understand main themes quickly. Although word clouds are recommended for use with full transcripts (2010), word clouds were presented for the present study using final coding of the transcripts. Moreover, by assigning certain categories of words different colors, deeper understanding was achieved. For instance, in the current study, positively-charged words were presented in green, neutral words will be presented in white, and negatively-charged words will be presented in red. This allowed the reader to understand some of the deeper context of what it means to be an athlete, as defined by athletes, without pouring through extended interview excerpts. For the researcher, creating a word cloud early in the analysis process was useful in future analysis (2010) to answer other questions related to how 
athletes identify the "athlete" identity and referring to it later in the analytic process acted as a measure of (dis)confirmation of findings.

Narrative accounts, using created nonfiction techniques (Caulley, 2008) generated from participants' own words, represented findings from the remaining research questions. Other methods of qualitative data presentation that focus on the researcher's interpretation of the data, using quotes from participants for support, tend to break up the flow of information and dissect the experience. Given that the current study used a narrative design, in large part to maintain the context of the data, using data presentation methods that maintain the context and flow was important. Waldron, Lynn, and Krane (2011) adopted creative nonfiction in their work on hazing in high school and collegiate male sport teams. The result is several compelling examples of hazing in sport that lead the reader to feel a part of the story, to intimately understand the details in ways that connect the reader, who has never and will never know the participant, to his lived experience. Specific techniques/considerations offered for using creative nonfiction in qualitative research (Caulley, 2008) include: purposeful openings that lead the reader to buy in and develop interest; scenes infused with detail, conversation, and metaphor that intensify the reading experience and act to "make the past present" (p. 429) as opposed to consolidated summaries that sap the experience of life and vitality; emphasis on the active voice by avoiding unnecessary grammatical crutches (i.e., "killer be's” (p. 434), unnecessary adverbs and adjectives); appropriate point of view, word choice, tone, sentence structure, litany, and prose rhythm that keeps the reader's engagement; and closings that are once expected and disappointing because the story has to end. Of critical importance when using creative nonfiction in qualitative inquiry is the necessity to maintain loyalty to the "truth" revealed by the data. Authors specifically caution against compression, or the consolidation of many events into a single, fictional account, 
for the sake of easier narration or to save space. However, compression that remains loyal to the data is encouraged. Recommendations for rigor include a commitment to the truth, fidelity to what is known and avoidance of conjecture, strategic compression, and member checking (Caulley, 2008). Creative nonfiction in narrative research is an intense endeavor through a thick forest explored by few, but by using written narrative to depict oral narrative, the researcher was better able to convey context and emotion, deepening the reader's understanding of, interest in, and empathy for the phenomena of interest. 
Dear Participant,

This letter is a request for you to take part in a research project to learn about the athlete identity. This project is being conducted by Ashley Cranney, MS in the Department of Sport Sciences at WVU with supervision of Dr. Jack Watson, a professor in the College of Physical Activity and Sport Science, for a Doctoral Degree in Sport and Exercise Psychology. Your participation in this project is greatly appreciated and will take approximately 1-2 hours for two interviews and 10-20 minutes to fill out the two questionnaires.

Your involvement in this project will be kept as confidential as legally possible. All data will be reported with identifying information removed. You must be 18 years of age or older to participate. Your participation is completely voluntary. You may skip any question that you do not wish to answer and you may discontinue at any time. Your class/team standing will not be affected if you decide either not to participate or to withdraw. West Virginia University's Institutional Review Board acknowledgement of this project is on file.

I hope that you will participate in this research project, as it could be beneficial in understanding the development of the athletic identity and how that identity is fulfilled. Thank you very much for your time. Should you have any questions about this letter or the research project, please feel free to contact Ashley Cranney by e-mail at Ashley.CokerCranney@mail.wvu.edu.

Thank you for your time and help with this project.

Sincerely,

Ashley Cranney, M.S. 


\section{Only Minimal Risk \\ Consent Information Form (without HIPAA)}

$\begin{array}{ll}\text { Principal Investigator } & \text { Ashley Coker-Cranney } \\ \text { Department } & \text { Sport and Exercise psychology } \\ \text { Protocol Number } & \text { Click here to enter text. } \\ \text { Study Title } & \text { Understanding Identity and Overconformity in Collegiate Wrestling } \\ \text { Co-Investigator(s) } & \text { Jack C. Watson, II, PhD } \\ \text { Sponsor (if any) } & \text { Click here to enter text. }\end{array}$

\section{Contact Persons}

Thank you for taking the time to consider participation in the following study.

In the event you experience any side effects or injury related to this research, you should contact Dr. at (304) _____ (After hours contact: Dr. at (304) ___ questions, concerns, or complaints about this research, you can contact $\mathrm{Dr}$. or Dr. at (304)

For information regarding your rights as a research subject, to discuss problems, concerns, or suggestions related to the research, to obtain information or offer input about the research, contact the Office of Research Integrity \& Compliance at (304) 293-7073.

In addition if you would like to discuss problems, concerns, have suggestions related to research, or would like to offer input about the research, contact the Office of Research Integrity and Compliance at 304-293-7073.

\section{Introduction}

You, have been asked to participate in this research study, which has been explained to you by the principle investigator. This study is being conducted by Ashley Cranney, a doctoral student in the Department of Sport and Exercise Psychology at West Virginia University.

\section{Purpose(s) of the Study}

This study is designed to develop deeper understanding of how athletes define what it means to be an athlete. You will be asked to share your life story as it relates to sport, sharing only what information you feel comfortable sharing. The information gathered from this study will assist Sport and Exercise Psychology Professionals better understand what it means to be an athlete and how athletes meet the expectations of that identity.

\section{Description of Procedures}

This study involves meeting with the principle investigator on two occasions and will take approximately 30-60 minutes each time for you to complete. During each meeting, you will speak with the principle investigator about your life 
experience with sport. She will provide you several prompts for information related to the purpose of the study. The information you choose to share on those occasions is up to you. You will also be asked to fill out two 7-item questionnaires regarding your relationship with your athletic identity and behaviors related to the fulfillment of that identity that you have witnessed throughout your sport career. Each will take approximately 5-15 minutes. You do not have to answer all the questions. You will have the opportunity to see the questionnaires before signing this consent form.

\section{Discomforts}

There are no known or expected risks from participating in this study, except for the mild frustration associated with answering the questions.

\section{Alternatives}

You do not have to participate in this study.

Alternatives that could be considered in your case include:

\section{Benefits}

You may not receive any direct benefit from this study. However, many other individuals who have participated in life story research have reported benefits related to increased self-awareness and understanding. The knowledge gained from this study may eventually benefit others.

\section{Financial Considerations}

There are no special fees for participating in this study.

\section{Confidentiality}

Any information about you that is obtained as a result of your participation in this research will be kept as confidential as legally possible. Your research records and test results, just like hospital records, may be subpoenaed by court order or may be inspected by the study sponsor or federal regulatory authorities (including the FDA if applicable) without your additional consent.

In addition, there are certain instances where the researcher is legally required to give information to the appropriate authorities. These would include mandatory reporting of infectious diseases, mandatory reporting of information about behavior that is imminently dangerous to your child or to others, such as suicide, child abuse, etc.

Audiotapes or videotapes will be kept locked up and will be destroyed as soon as possible after the research is finished. In any publications that result from this research, neither your name nor any information from which you might be identified will be published without your consent.

\section{Voluntary Participation}

Participation in this study is voluntary. You are free to withdraw your consent to participate in this study at any time.

Refusal to participate or withdrawal will not affect your class standing, grades, or participation on the team and will involve no penalty to you.

In the event new information becomes available that may affect your willingness to participate in this study, this 
information will be given to you so that you can make an informed decision about whether or not to continue your participation.

You have been given the opportunity to ask questions about the research, and you have received answers concerning areas you did not understand.

Upon signing this form, you will receive a copy.

I willingly consent to participate in this research.

\section{Signatures}

Signature of Subject

Printed Name Date Time

The participant has had the opportunity to have questions addressed. The participant willingly agrees to be in the study.

Signature of Investigator or Co-Investigator

Printed Name Date Time




\section{Life Story Interview Guide}

This is an interview about the story of your life in sport. As a social scientist, I am interested in hearing your story, including parts of the past as you remember them and the future as you imagine it. The story is selective; it does not include everything that has ever happened to you. Instead, I will ask you to focus on a few key things in your life - a few key scenes, characters, and ideas. There are no right or wrong answers to my questions. Instead, your task is simply to tell me about some of the most important things that have happened in your life and how you imagine your life developing in the future. I will guide you through the interview so that we finish it all in about two hours or less.

Please know that my purpose in doing this interview is not to figure out what is wrong with you or to do some kind of deep clinical analysis! Nor should you think of this interview as a "therapy session" of some kind. The interview is for research purposes only, and its main goal is simply to hear your story. Everything you say is voluntary, anonymous, and confidential.

I think you will enjoy the interview. Do you have any questions?

\begin{tabular}{|c|c|}
\hline Life Chapters & $\begin{array}{l}\text { - Think of your life in sport as if it were a book-a novel } \\
\text { with chapters. } \\
\text { What would the chapters be? } \\
\text { - Divide your sport life story into its main chapters, and } \\
\text { for each chapter provide a title and brief plot summary. } \\
\text { - Explain what marks the end of one chapter and the } \\
\text { beginning of the next. }\end{array}$ \\
\hline $\begin{array}{l}\text { Key Scenes } \\
\text { High Point } \\
\text { Low Point } \\
\text { Turning Point } \\
\text { Positive Childhood Scene } \\
\text { Negative Childhood Scene } \\
\text { Vivid Adolescent Scene } \\
\text { Vivid Adult Scene } \\
\text { One Other Important } \\
\text { Scene }\end{array}$ & $\begin{array}{l}\text { - Focus on a few specific moments or episodes that } \\
\text { stand out as being especially memorable or important } \\
\text { in your life story in sport. } \\
\text { - For each scene, describe in detail what happened, who } \\
\text { was there, what you were thinking and feeling in the } \\
\text { scene, and what significance you believe the scene has } \\
\text { in the context of your entire life story in sport. } \\
\text { - Why do you think you chose this scene? } \\
\text { What might the scene say about who you were or are? }\end{array}$ \\
\hline Life Challenge & $\begin{array}{l}\text { - Identify the most important challenge, struggle, or } \\
\text { conflict you have faced in your life in sport. } \\
\text { Describe what the challenge is, how it came to be, and } \\
\text { how you have tried to address it or cope with it. }\end{array}$ \\
\hline Future Script & $\begin{array}{l}\text { - What does the next chapter of your life story look like? } \\
\text { - } \quad \text { fescribe where you think your life is headed in the } \\
\text { - What are your main goals for the future? } \\
\text { - How do you plan to achieve those goals? }\end{array}$ \\
\hline $\begin{array}{l}\text { Ideological Setting } \\
\text { Religious } \\
\text { Political } \\
\text { Most Important Value }\end{array}$ & $\begin{array}{l}\text { - Consider here your most important beliefs and values } \\
\text { about life and the world in sport. } \\
\text { - First, describe any values and beliefs that you consider } \\
\text { - } \quad \text { bow important for your life in sport. } \\
\text { - Finally, what develop those values and beliefs? } \\
\text { value in life? } \\
\text { - Why? }\end{array}$ \\
\hline Life Theme & $\begin{array}{l}\text { - Thinking back over what you have said in this interview, } \\
\text { do you see a theme or motif that runs through the story } \\
\text { of your life in sport? } \\
\text { - What might it be? }\end{array}$ \\
\hline
\end{tabular}

Note: Introduction adapted from McAdams (2008) The Life Story Interview, The Foley Center for the Study of Lives at Northwestern University. Questions adapted from McAdams and Guo (2014). 


\section{AIMS \\ (Brewer \& Cornelius, 2001)}

Please circle the number that best reflects the extent to which you agree or disagree with each statement regarding your sport participation.

1. I consider myself an athlete.

$\begin{array}{lllllllll}\begin{array}{l}\text { Strongly } \\ \text { Disagree }\end{array} & 1 & 2 & 3 & 4 & 5 & 6 & 7 & \begin{array}{l}\text { Strongly } \\ \text { Agree }\end{array}\end{array}$

2. I have many goals related to sport.

$\begin{array}{lllllllll}\begin{array}{l}\text { Strongly } \\ \text { Disagree }\end{array} & 1 & 2 & 3 & 4 & 5 & 6 & 7 & \begin{array}{l}\text { Strongly } \\ \text { Agree }\end{array}\end{array}$

3. Most of my friends are athletes.

$\begin{array}{lllllllll}\begin{array}{l}\text { Strongly } \\ \text { Disagree }\end{array} & 1 & 2 & 3 & 4 & 5 & 6 & 7 & \begin{array}{l}\text { Strongly } \\ \text { Agree }\end{array}\end{array}$

4. Sport is the most important part of my life.

$\begin{array}{lllllllll}\begin{array}{l}\text { Strongly } \\ \text { Disagree }\end{array} & 1 & 2 & 3 & 4 & 5 & 6 & 7 & \begin{array}{l}\text { Strongly } \\ \text { Agree }\end{array}\end{array}$

5. I spend more time thinking about sport than anything else.

$\begin{array}{lllllllll}\begin{array}{l}\text { Strongly } \\ \text { Disagree }\end{array} & 1 & 2 & 3 & 4 & 5 & 6 & 7 & \begin{array}{l}\text { Strongly } \\ \text { Agree }\end{array}\end{array}$

6. I feel bad about myself when I do poorly in sport.

$\begin{array}{lllllllll}\begin{array}{l}\text { Strongly } \\ \text { Disagree }\end{array} & 1 & 2 & 3 & 4 & 5 & 6 & 7 & \begin{array}{l}\text { Strongly } \\ \text { Agree }\end{array}\end{array}$

7. I would be very depressed if I were injured and could not compete in sport.

$\begin{array}{lllllllll}\begin{array}{l}\text { Strongly } \\ \text { Disagree }\end{array} & 1 & 2 & 3 & 4 & 5 & 6 & 7 & \begin{array}{l}\text { Strongly } \\ \text { Agree }\end{array}\end{array}$




\section{SMOSS}

(Allen, 2003)

Please circle the number that best reflects the extent to which you agree or disagree with each statement regarding your sport participation.

I feel things have gone well in my sport when...

1. Others tell me I have performed well.

$\begin{array}{lllllll}\begin{array}{l}\text { Strongly } \\ \text { Disagree }\end{array} & 1 & 2 & 3 & 4 & 5 & \begin{array}{l}\text { Strongly } \\ \text { Agree }\end{array}\end{array}$

2. I make some good friends on the team.

$\begin{array}{lllllll}\begin{array}{l}\text { Strongly } \\ \text { Disagree }\end{array} & 1 & 2 & 3 & 4 & 5 & \begin{array}{l}\text { Strongly } \\ \text { Agree }\end{array}\end{array}$

3. I belong to the popular group in the team.

$\begin{array}{lllllll}\begin{array}{l}\text { Strongly } \\ \text { Disagree }\end{array} & 1 & 2 & 3 & 4 & 5 & \begin{array}{l}\text { Strongly } \\ \text { Agree }\end{array}\end{array}$

4. My teammates and I have a laugh together.

$\begin{array}{lllllll}\begin{array}{l}\text { Strongly } \\ \text { Disagree }\end{array} & 1 & 2 & 3 & 4 & 5 & \begin{array}{l}\text { Strongly } \\ \text { Agree }\end{array}\end{array}$

5. I am the center of attention.

$\begin{array}{lllllll}\begin{array}{l}\text { Strongly } \\ \text { Disagree }\end{array} & 1 & 2 & 3 & 4 & 5 & \begin{array}{l}\text { Strongly } \\ \text { Agree }\end{array}\end{array}$

6. I make new friends who I socialize with outside sport.

$\begin{array}{lllllll}\begin{array}{l}\text { Strongly } \\ \text { Disagree }\end{array} & 1 & 2 & 3 & 4 & 5 & \begin{array}{l}\text { Strongly } \\ \text { Agree }\end{array}\end{array}$

7. I have fun with the others on my team.

$\begin{array}{lllllll}\begin{array}{l}\text { Strongly } \\ \text { Disagree }\end{array} & 1 & 2 & 3 & 4 & 5 & \begin{array}{l}\text { Strongly } \\ \text { Agree }\end{array}\end{array}$


Ifeel things have gone well in my sport when...

8. I am part of the "in" crowd.

$\begin{array}{lllllll}\begin{array}{l}\text { Strongly } \\ \text { Disagree }\end{array} & 1 & 2 & 3 & 4 & 5 & \begin{array}{l}\text { Strongly } \\ \text { Agree }\end{array}\end{array}$

9. Other kids think I'm really good at sport.

$\begin{array}{lllllll}\begin{array}{l}\text { Strongly } \\ \text { Disagree }\end{array} & 1 & 2 & 3 & 4 & 5 & \begin{array}{l}\text { Strongly } \\ \text { Agree }\end{array}\end{array}$

10. I receive recognition from others for my accomplishments.

$\begin{array}{lllllll}\begin{array}{l}\text { Strongly } \\ \text { Disagree }\end{array} & 1 & 2 & 3 & 4 & 5 & \begin{array}{l}\text { Strongly } \\ \text { Agree }\end{array}\end{array}$

11. Spending time with the other players is enjoyable.

$\begin{array}{llllllll}\begin{array}{l}\text { Strongly } \\ \text { Disagree }\end{array} & 1 & 2 & 3 & 4 & 5 & \begin{array}{l}\text { Strongly } \\ \text { Agree }\end{array}\end{array}$

12. I become friends with some of the others in my sport.

$\begin{array}{llllllll}\begin{array}{l}\text { Strongly } \\ \text { Disagree }\end{array} & 1 & 2 & 3 & 4 & 5 & \begin{array}{l}\text { Strongly } \\ \text { Agree }\end{array}\end{array}$

13. Others are impressed by my sporting ability.

$\begin{array}{lllllll}\begin{array}{l}\text { Strongly } \\ \text { Disagree }\end{array} & 1 & 2 & 3 & 4 & 5 & \begin{array}{l}\text { Strongly } \\ \text { Agree }\end{array}\end{array}$

14. I am one of the more popular players.

$\begin{array}{lllllll}\begin{array}{l}\text { Strongly } \\ \text { Disagree }\end{array} & 1 & 2 & 3 & 4 & 5 & \begin{array}{l}\text { Strongly } \\ \text { Agree }\end{array}\end{array}$

15. Just hanging out with the others is fun.

$\begin{array}{lllllll}\begin{array}{l}\text { Strongly } \\ \text { Disagree }\end{array} & 1 & 2 & 3 & 4 & 5 & \begin{array}{l}\text { Strongly } \\ \text { Agree }\end{array}\end{array}$




\section{Semi-Structured Interview Guide}

1. What does it look like to be an athlete? How do you define it?

2. How did you learn what it means to be an athlete?

3. What things are expected of people who are identified as "athletes?"

4. How do people fulfill those expectations? How did/do you?

5. Do you think that some people go too far? Have you? How?

6. Why do you think some people go too far? Why did you?

7. Is it a good or bad thing to go too far? Why? 


\section{Written Expression Template}

What do athletes have to "do" to be considered athletes?

How do you think athletes sometimes go "too far" to try and prove that they are athletes?

Why do you think athletes sometimes go "too far?"

Have you ever known anyone who went "too far" trying to fulfill the role of athlete?

What did he/she do that made you think he/she went "too far?" 
To what extent have you seen athletes do the following things? $(1=$ rarely, $5=$ often $)$

Practice/compete while injured/in pain.

$\begin{array}{lllll}1 & 2 & 3 & 4 & 5\end{array}$

In what ways have you seen athletes manage their pain/injury in order to keep playing?

Manage weight using unhealthy methods.

$\begin{array}{lllll}1 & 2 & 3 & 4 & 5\end{array}$

In what ways have you seen athletes manage their weight in unhealthy ways?

Use performance-enhancing substances.

$\begin{array}{lllll}1 & 2 & 3 & 4 & 5\end{array}$

In what ways have you seen athletes use substances to improve their performance?

Engage in excessive violence (e.g., trying to hurt an opponent) when practicing/competing.

$\begin{array}{lllll}1 & 2 & 3 & 4 & 5\end{array}$

In what ways have you seen athletes use excessive violence in practice/competition?

Engage in hazing practices.

$\begin{array}{lllll}1 & 2 & 3 & 4 & 5\end{array}$

In what ways have you seen athletes engage in hazing practices? For what purpose(s)? 


\section{Provisional Coding Framework}

PROVISIONAL CODES

Sport Ethic (Hughes \& Coakley, 1991)

- Striving for distinction (DISTINCTION): seeking to set oneself apart from others and achieve recognition for doing so

- Sacrificing for The Game (SACRIFICE): putting the sport above all else, prioritizing it over relationships, academics, work, etc

- Playing through pain and injury (PLAY THROUGH): playing in pain to prove commitment

- Accepting no limits in the quest for greatness (NO LIMITS): refusing to acknowledge "can't," continuing practices regardless of failure, perseverance in the face of extreme adversity

Reasons People Overconform (Coakley, 2015; Waldron \& Kowalski, 2009)

- Keep Playing (KEEP PLAYING): seeking to continue involvement in sport as long as possible

- Low Self-Esteem (SE): athletes with low self-esteem establish self-worth via peer acceptance

- Proving Masculinity (PROVE): being an athlete and being a man become the same identity

- Social-Approval Goal Orientation (SOC-APPROVE): athletes high in social approval goal orientation require the acceptance of their peers to feel good and successful in their sport experiences

Identity Construction and Confirmation (Donnelly \& Young, 1988)

- Presocialization (PRESOC): prior to joining the team, learn about sports/the sport, learn about the athletes in sports/the sport, spend time with others at/watching/talking about sporting events; all learning is from outside perspective

- Selection/Recruitment (JOIN): tryout or recruited to tryout/join team by coaches, friends on team, established athletes, admin

- Socialization (SOC): learn about what sport is from insiders/inside perspective; oriented to expectations of what it means to be an athlete, communicated specific behaviors to gain acceptance

- Acceptance (CONFIRM): accepted by peer athletes/coaches/spectators/media as athlete

Overconforming behaviors from the literature

- Disordered eating (DE): weight loss/gain methods (e.g., extreme dieting, saunas, diuretics, bingeing, additional workouts) to make weight

- Refusal to acknowledge illness/injury (INJURY): returning from injury too quickly, refusing to seek help for injury, ignoring injury, playing ill, sacrificing bodily sensations of pain (e.g., from restricted diet or overexercise) to play

- Pain management techniques (PAIN MGMT): routine painkiller injections, routine NSAID use, icing/heating pre/post practice/competition

- Performance enhancing substances (PES): anabolic steroids, conditioning at high 
altitude

- Excessive violence (INC. VIOLENCE): intentionally trying to hurt a player for points, taking cheap shots to ensure victory

- Overtraining (OVERTRAIN): training on own, in addition to team; practicing extra hours

- Underrecovery (UNDERRECOVER): hit the wall due to minimal recovery, body shuts down, trouble thinking

- Hazing (HAZING): perpetuating, complying, or receiving hazing rituals in an effort to fit in with the team

- Cheating (CHEATING): athletes who overconform may be more likely to cheat in an effort to continue playing

Additional codes generated from pilot interview

- Competition/Winning (WIN): who wins matters; winning is an essential component of sport

- Fun (FUN): sports are fun

- Coach (COACH): coaches are highly influential in the socialization and decisionmaking processes athletes encounter

- Acceptance/Caring (ACCEPT): athletes need to feel cared for by significant others

- Discipline/Commitment (DISCIPLINE): athletes are expected to work hard and exercise discipline as part of the sport subculture

- Honesty/Respect (HONESTY): athletes should respect their teammates and opponents, as well as the integrity of the game

- Bullying (BULLY): bullying is a central part of the sport subculture

- Athlete (ATHLETE): characteristics of the concept of "athlete" 
APPENDIX F 


\section{Personal Background}

Growing up, I participated in any sport I could convince my parents to sign me up for. Early on, I enrolled in gymnastics, soccer, and baseball. As I got older, my mother told me she was going to enroll me in dance classes because they would help me become a better athlete. Although I resisted at first, I found that I enjoyed them and signed up for as many as I could. When I reached middle school, I started tumbling classes and playing volleyball and basketball, experimented with track and field. As dance and tumbling became a larger part of my extracurricular activities, I dropped track and field and basketball to focus on dance, tumbling, and volleyball. By the time I reached high school, I broke my ankle just prior to volleyball season, which ended my volleyball career, so I focused all of my energy on dance and tumbling. After I healed, I started cheerleading. By my senior year, I resumed my love affair with track and field jumping events, and continued dancing, tumbling, and cheerleading. Through it all, my parents had one rule: if I signed up for it, I must finish the season. I was never allowed to skip a single practice or competition unless I was visibly, unquestionably incapacitated; sport teaches values and skipping practice or quitting, demonstrates poor commitment and dependability, which would not be tolerated.

Although I had always identified myself as an athlete, I began embracing the identity of dancer, which guided the majority of my decisions. I became so immersed in the world of dance and cheerleading that I would do anything to continue participation. I thought that my coaches walked on water and would do anything to please them. If I was told to diet, I did it. At one point, I spent several months eating a banana and drinking a bottle of apple juice to hold me through the day until I returned home at 10:00pm to have my bowl of cereal and go to sleep. I believed that holding true to my diet would surely separate me from my peers, distinguishing me 
from them by proving my commitment to dance and cheerleading. If I was told to lose weight, I did so and then some. I put in extra workouts and stopped eating the banana, excited at how much weight fell away. By the time track season began, I found myself too weak to perform, so I started eating a corndog at lunch to give me the energy I needed to jump. When my friends voiced their concerns, I added a second corndog and ate the breading. Through the many injuries I sustained, from sprained ankles to torn rotator cuffs to a sprained neck, I kept competing. I would take Aleve and ice each night and ignore the pain each day. My coaches praised me, my peers admired me, and the judges awarded my performances. I was certain that all athletes, or at least those who were really committed, who really cared, who were dependable, did the same types of things.

By the time I got to college, I focused only on dancing for my team. I took additional dance classes to avoid the dreaded "freshman fifteen" and continue to practice my skills. I was disappointed that my college coach was severely deficient in the coaching area, so I turned my attention to pleasing my captain. After all, if I wanted the best positions and the best parts, she was the one I had to please. By my senior year, my "coach" left us, leaving me, the captain, in charge of the program. Without someone to watch my excessive dieting and exercising behavior, I sustained several severe back and neck injuries that hindered my performance. However, I remembered that there is no excuse to miss practice, and my teammates relied on me to maintain the program, so I continued moving forward, using my school breaks to heal. When I blew my knee out and had surgery, I gave myself three weeks to rest before returning to full practice. In my mind, that is what real athletes, those who really wanted it, did. A few weeks after I returned, I tore my rotator cuff again. Shortly after, I strained several muscles in my back that made walking one of the most painful activities I could imagine. Near the end of the season, I got very 
sick, breaking my ribs by simply coughing. At that point, my parents began expressing their concern, my now husband asked me to quit at the end of the year, and my "advisor" recommended I consider hanging up my dancing shoes and applying to become the new coach. At first, I resisted. I was an athlete, not a coach. However, after I accidentally overdosed on cough syrup and was rushed to urgent care by the athletic training staff for uncontrollable muscle spasms, I realized I was not in the mental nor physical state to continue dancing. My body was telling me it was time to call it quits, and I could not silence it any longer.

After my last season, I was hired as the new coach. I swore, when I was hired, that I would not pressure athletes to lose weight and that if they were injured, they were benched until the athletic trainer told me, personally, that they were ready to return. For a few years, I felt fulfilled enough. However, the hole left inside of me from losing me athletic identity-from having it stolen from me-was too great. I had trouble exercising because my old injuries caused too much pain. I avoided situations where I might dance because the confrontation that I was no longer the athlete I had been was too great. In fact, I still avoid situations that call for social dancing for the same reason.

I had to find something to fill the hole, so I poured myself, everything I had, into being the best coach I could be. I spent hours and denied myself sleep to make certain my athletes always had everything they needed. In doing so, I continued denying myself basic needs. So, I finally made the decision to go to graduate school. The anticipation that my connection with the sport world was completely severed-I could not even pretend that coaching kept me involvedled me to search for another way to distinguish myself. In my search, I found that my only hope was to become the best graduate student in recent history. As with my identity as athlete, my identity as graduate student was equally important, requiring me to utilize the same discipline, 
determination, and commitment to set myself apart.

During my course of study, I learned about overconformity to the sport ethic. Everything about it spoke to me. I saw myself in the framework. I heard myself in the stories I read about athletes who put their bodies on the line for approval from their coaches and recognition from their peers. I became resolved to understand how the messages about what it takes to be a true athlete are conveyed, how athletes become willing to do anything for the recognition as athlete, the role coaches play in athletes' overconforming behaviors, how to explain the fear of losing such a prized identity, and, ultimately, to find a way to change the culture of sport. However, the part of me that still considers myself ultra-athlete, ultra-competitive, ultra-determined still wrestles with the idea that we should not praise overconformity. After all, by taking away the ethic of demonstrating commitment to the athlete identity, sport is no longer sport — at least according to the athlete in me. Even today, it is still a daily struggle not to feel the need to prove myself, to recognize that I am worthy for who I am, not the unquestioning commitment I feel compelled to display to others. As much as sport gave me, it also stole a great deal from me. It is my hope that through my research, I can help other athletes avoid the mental and physical pain I experienced then, and continue to experience now. 
APPENDIX G 


\section{Interview Pilot Study Results}

A pilot interview was conducted with an ex-wrestler to gain a better understanding of researcher biases and test methodological choices. The interview (i.e., combined life story interview and semi-structured interview) with "Dale," a Caucasian male in emerging adulthood who wrestled competitively in three distinct programs, was conducted over the span of 50 minutes and 37 seconds. Written data for the pilot participant were not gathered given that the primary goals of pilot data collection were to highlight researcher biases, guide semi-structured interview protocol, and refine provisional codes. All data from the pilot interview were analyzed using provisional coding, to give the researcher experience using this method and address the influence her biases had on data analysis. Presented below are a word cloud that addressed research question one and a narrative to address research questions two, three, and four.

From the pilot interview, the researcher was able to address each research question, albeit some with more depth than others. Specifically, "Dale" provided great depth of detail regarding how he identified the term "athlete." He also provided great detail regarding how social acceptance of his athlete identity occurred for him, highlighting the importance of his coaches and media rather than peer acceptance of the "athlete" identity. The difficulty faced by the researcher to address the third research question indicated that the researcher should include more focused questions to illicit participants' responses during the semi-structured interview, specifically as related to the participant's recognized meaning of the athlete identity.

Likewise, although the participant addressed several instances where he met the expectations of the "athlete" identity (research question four), more detail is needed to understand those behaviors. The second written exercise is proposed to help with this issue. Using the FCE principle, the researcher for the current study hopes that by prompting the 
participant to identify overconforming behaviors in others through a brief written exercise, he may be more willing to address those in himself given that his "observation" of overconforming behaviors in others may support his own engagement. If that is not the case, the FCE still provides a compelling reason to assume that the participants' accounts of observed overconforming behaviors may reflect his own, even if he is unwilling to share that information during the interview.

"Dale's" Definition of the Athlete Identity. "Dale" was presented with the following word cloud, based on his responses in the interview (Figure 7). Although previous qualitative research using word clouds entered an entire transcript into the word cloud generator (McNaught \& Lam, 2010), the word cloud generated for this study was based upon round one coding of the participant interviews for precision and clarity. The size of each word (with the exception of "athlete") indicates the frequency with which that word was referred to, and coded, to answer the first research question of how athletes define the "athlete" identity. Athlete characteristics/foci highlighted in green represent those characteristics/foci he spoke fondly about. Those highlighted in white were sometimes referred to positively and sometimes negatively. Words highlighted in red represent characteristics/foci that "Dale" referred to negatively.

By sharing the resulting word cloud with "Dale," he was able to check the accuracy of the researcher's analysis and interpretation. After presenting "Dale" with the word cloud and explaining what it represented and how it was represented, he offered no clarifications or changes. He reported believing it was an accurate representation of how he defined athlete.

"Dale's" Narrative. The following narrative is presented to address research questions two, three and four. It is presented in two consecutive parts. In the first, the researcher sets the stage for the story using "Dale's" greatest struggle, inviting the reader to understand the context 
of "Dale's" experience and deeply connect with that experience. Following the opening scene, a

monologue, compiled using mainly "Dale's" direct words, is presented to illustrate how he

learned about sport, was socialized into the role of athlete, and met his own and significant

others' expectations of the role of athlete.

He lived in the same small town his whole life. He played hide and seek in the field with his friends, armed with only a four wheeler and a head light. He played crossing the border and $a$ litany of other night games with the friends he had his whole life. The scrawny, shy kid who never really bothered anyone endured bullying and been in trouble at school for fighting back, but he continued wrestling anyway. He became good, very good. But now, Dale had a choice to make. His family was moving away. His assistant wrestling coach offered to let Dale live at his house for his last two years of school and continue wrestling with the team. The offer was alluring. It made him feel special. Important. Wrestling meant something to Dale. The program had won the last seven State titles in as many years and Dale was a part of that history. He was going to be on Varsity the next year. When his parents told him about the move, Dale didn't take it well. He was dominant in a dominant wrestling program but his parents wanted to move him away from that program, away from the coaches who cared enough for him to take him in and make him something great. It had given him confidence. Choosing to move away with his family was not an easy choice to make and in a new wrestling program with coaches who didn't care, who seemed like bullies, who weren't good wrestlers themselves, and a community that didn't support wrestling, Dale would look back on his choice and wonder if it was the right one to make.

Growing up, I spent a lot of time with my dad. My dad was the hardest worker I've ever known in my whole life. He would get up, work all day, come home, work 'til dark, get up, do it all again the next day, do it on Saturdays and Sundays. He'd include us. We'd haul firewood, haul hay, pick rocks. When we were with him, we were working, and we were working hard. He taught us that if you start something, you finish it.

To have fun, I'd play night games. Those were some of the best times of my life. We'd play crossing the border and hide and go seek with a four wheeler and a headlight. It was just fun to do stuff with friends, causing mischief. One time, I remember having a good time with one of my friends and my dad almost caught us. We were jumping and doing flips and backflips on the trampoline. We had pulled it over to the fence, so we were jumping off of the camper and onto the tramp. Then we saw my dad come around the corner in his truck. We lived in a pretty open space, so we knew there were still a few more minutes before he would finish the drive to our house. We knew we had to get inside and be in bed, so we hit the tramp, bounced off to the ground, then jumped up the stairs to my bedroom, hiding before he got halfway down the road. I think he knew, but he pretended he didn't see us. That was fun.

I lived in a pretty small town, so there weren't a lot of little leagues and things where I could learn to play sports. When I was started third grade, things really started to change. Where I went to school, when you hit the old side of the elementary school, then it mattered whether you won or lost. We kept score. It was fun. Winning means you get to keep playing, winning means you stay on the court. If you want to keep playing, you gotta win. There's always a winner and a loser. You play, you keep score, and winning is fun. I really don't believe in the 'everybody gets a trophy' thing that this world's turned into. There's always a winner and a loser. You can't have 
the "hey, we all came out to have fun" attitude. As kids, it might make you more competitive, but so be it. Eventually, you have to keep score. You have to lose to know what winning is worth.

When I was in junior high school, I played football and basketball and wrestled, but basketball and wrestling have the same season, so I had to pick one. Sure, there were hard times. There was one kid who bullied me in junior high because I was scrawny and shy. I think that's why he picked on me. He was a year older than me and a hell of a lot bigger than me. I don't know, maybe he got picked on or something. I think fighting and bullying comes from your upbringing. There's hazing in sport, but there's also assholes in the world. Bullies would pick on younger kids who did not have an older brother on the team. I got taped up once. I had my wrists and ankles taped together and I rolled off the seat onto a corner and knocked myself out. That was not a fun time. I think the kid wanted me to quit, but I was a good wrestler, so we had some battles.

When I started high school, I tried out for the basketball team and got cut. I wasn't sure what I was going to do, but the wrestling coach came to me and was like, "Hey, I watched you all through junior high and I want you to come wrestle for me." The season had already started and he drug me out there and made me start wrestling. It made me feel really special that he came and got me. It was really a turning point in my life. He made me a really good wrestler. He taught me to care about being physical, strong, in shape, and hard working. He really taught us how to work hard. Practice was hard and it was exhausting and we would do it until somebody puked, but it was fun. There aren't many sports where you compete for only six minutes and when you get done, you are one hundred percent exhausted. Every muscle in your entire body is exhausted and you are just done. The physical shape you have to be in for that is just unbelievable.

My coach had a really good program, they won States seven years in a row. I was just a scrawny little kid, so to make that effort to get me to come out, showed me that he was just a good guy. He cared. He didn't just have us there to win, even though it was a winning program, and we worked hard for him. There's no cutting in wrestling, but he wouldn't weed out the people who couldn't handle it. He would encourage us to stay the entire time and compete. There were awards for winning practice drills. It's just how he made sport fun. The coaches would sit there and encourage us like it was a match that actually mattered. A coach should motivate you to do the best that you can. I feel like I had a couple of coaches like that; they definitely impacted my life.

A few years after I started wrestling for my high school, my parents said we were moving. I was becoming very good, very dominant in a very dominant wrestling program. I was probably going to be on Varsity my next year, and they wanted to move me to a school that wasn't very good. It was probably my biggest challenge growing up. We had lived in the same place my whole life, and I was getting really good at wrestling, so when my parents said we were moving, I didn't take it well. I didn't want to leave my coaches and my program. So the assistant coach offered to let me stay at his house when my parents moved and finish my last two years wrestling with him. It made me feel special, important, like he really cared. He was my favorite coach, a really good coach. He still is my favorite coach. His offer gave me confidence. To have somebody care enough to make that offer was cool. Choosing to move with my family was a big struggle for me. It was probably the biggest struggle I had growing up.

When we moved to the new program, I was cynical. The new coach wasn't a great coach and it was a terrible program. He was not a very good wrestler. He seemed more like he wanted to bully us. There wasn't any support for the new program, either. At my old school, people actually cared about wrestling and would come watch, but at my new school, parents would come and that was it. I really missed my old program and my old coaches.

When I was a senior, I competed in State wrestling again. I got interviewed by the paper 
and they started following me. I had beaten my rival in Districts. I'm a very competitive person and beating him was a goal of mine so I really felt good accomplishing it. I felt like I was good and I was getting recognition for it. States were fun when we were winning; who wins matters. It was hard sometimes, too. I can remember running laps because they had free chocolate milk. You know what's really great at a wrestling match when you have to make weight? Free chocolate milk. Anyway, it was still my high point in sport. I was really close with some of my teammates. I actually hurt my ankle jumping up and down for my friend who beat his rival there. So I had to compete my last match on a hurt ankle. I found out later that I had a hairline fracture. I wrestled hard, though. I did well.

For me, sport was over after high school, but it taught me a lot. You have to finish what you start. You have to work hard, and you have to do it the right way. I don't believe in cheating, I would rather lose than cheat. You have to sacrifice. Wouldn't you want someone who sacrificed to teach your kid to sacrifice? It was over for me after high school, but I still enjoy sports. It's just fun playing morning basketball or after work with your friends. I still play intramural sports. I got a safety once in intramural football. A safety isn't really something that should be an option in intramural football, and I did that, but who cares about someone getting a safety in flag football? It's just intramurals, but it is fun. Looking forward, I want to play sports with my kids - pretty much any little sport thing they want to do. I can't wait, I'm so excited. I believe that if you play sports, you'll be able to walk better when you get older, and you'll learn to work hard and honor your commitments and do things the right way, so I'll teach my kids to play. I want them to know the value of hard work, commitment, and being in shape.

Brief Discussion of Pilot Results. In answer to the first research question, how athletes define that athlete identity, "Dale" talked about the importance of hard work and winning. He spoke of the need to lose to understand winning and that winning is fun. He spoke about the necessity that athletes are in shape, even contrasting the type of shape one must be in to wrestle with "easier" sports like basketball and football. Dale also provided information about the "athlete" identity that he did not agree with (i.e., bullying), but that seemed to be a part of the identity of some athletes he knew growing up. He discussed how dealing with pain and injury are a part of sport and that, although negative experiences, demonstrate athlete commitment.

To address research question two, pilot data supported the proposed stages of identity development presented by Donnelly and Young (1988), at least in relationship to coach acceptance of athlete identity. Specifically, "Dale" was oriented to the value of hard work in the pre-socialization phase. After being recruited to sport, he learned about what it meant to be a 
wrestler largely from his coaches. During the socialization phase, he was strongly oriented to an overall norm he labelled "commitment", demonstrated by working hard and competing, regardless of skill level, which reinforced earlier values instilled by his father. During socialization, he was oriented to sport-specific norms of striving for distinction, playing through pain and injury, sacrificing for the game, and accepting no limits (Hughes \& Coakley, 1991), although he did not use those words. Thus, Dale learned to measure his athletic identity on the basis of his success in a successful program and recognition from coaches and media.

Assessing what meaning the athlete identity held for "Dale" was more difficult in the pilot study. Specifically, at different chapters in his life, sport had served different functions, making a coherent understanding of its importance throughout his life difficult to discern. During his childhood, "Dale" discussed how the meaning of sport was centered on having fun with his friends. However, he indicated that once he entered the third grade, the emphasis of sport became the importance of winning, stating that "then it matter[ed] whether you win or lose." This shift related to his socialization that striving for distinction by beating his peers in competition is an important function of sport. Through his young teen and high school years, entitled "Puberty" and "Peak," respectively, the importance of winning, and what that meant for "Dale's" feelings of being "special" and "important," was amplified. In fact, he experienced both his high and his low points during his "Peak" chapter when he attended the State wrestling tournament and was interviewed by the paper, but then injured his ankle before his final match. When asked about the turning point in his life, "Dale" discussed the importance of his recruitment to high school wrestling by the coach. His greatest challenge/struggle/conflict also came during the same chapter when he had to make the decision to sacrifice living in the same city as his family or moving away from the distinguished wrestling program he had become a part of. In his final 
chapter, post-formal sport, "Dale" returned to a fun-based meaning of sport, where he discussed the importance of spending time with coworkers watching or playing sport. Interestingly, the importance of winning in sport seemed to stay with "Dale" as he also discussed several instances where winning has been important in his post-formal sport experiences. The finding that many of "Dale's" important life events occurred during the socialization and acceptance stages in "Dale's" athlete identity development process (i.e., "Peak") indicated that those experiences had a large impact on the meaning he ascribed the athlete identity and its influence on his life, in general.

Dale's narrative revealed an interesting tension related to the role he believed sport played in his development and the purpose it served in his life. Although he mentioned that fun was an important function of sport in his childhood and after the conclusion of his formal involvement in wrestling, he seemed to struggle with whether it was more important than winning. On several occasions, he contradicted himself, at once discussing how sport is "just fun" and then at other times that it was about winning. Particularly, although he discussed the importance of having fun in sport when one is young, he also vehemently opposed the idea of participation trophies, stating "I don't believe in the 'everybody gets a trophy' thing that this world's turned into." This is not surprising given his endorsement of the sport norm to strive for distinction. If everyone gets a trophy for showing up, how does one distinguish himself?

In response to how he fulfilled the expectations of the athletic identity, "Dale" discussed several behaviors generally considered to be indicative of overconformity. Specifically, he discussed running laps at a State wrestling meet because free chocolate milk was available to wrestlers. When sharing this experience, he alluded to the sport pressure to maintain a specific weight and the general acceptance in the wrestling culture that last minute weight management 
techniques are widely acceptable. Moreover, in relation to his high point in sport, "Dale" talked about how he competed his final match at the State wrestling tournament on a hurt ankle he earned earlier in the tournament, indicating his adherence to the norm that athletes play through pain and injury. However, rather than dwelling on the fact that his ankle was hurt, "Dale" refocused on the "high" he felt beating his rival at Districts earlier that season, who he had not beaten in previous matches. The theme that "Dale" often strove for distinction permeated his entire interview, reflecting the importance this norm had in his understanding of the wrestling subculture and athlete identity.

A considerable piece of "Dale's" life story addressed what he referred to as hazing and bullying. He cited specific incidents where he endured hazing and bullying practices, normalizing them as part of the sport subculture, explaining that it was expected to get "picked on" if one did not have an older brother on the team. Although he tolerated several bullying experiences, "Dale" also discussed how he eventually rejected the team norm and fought back against his attacker after he was taped up and knocked out. Therefore, while "Dale" expressed an agreement with several sport norms (e.g., as he modified his eating and exercise habits to lose weight, competed while significantly injured, considered living without his family in favor of continuing participation with a distinguished program, experienced great pride when publicly recognized for his accomplishments, trained until he puked, and kept practicing and competing to beat someone he had been previously unable to beat), he also took exception with those norms that skewed the playing surface (e.g., cheating, hazing/bullying). As a result, "Dale" provided evidence that whereas some athletes may engage in overconforming behaviors to demonstrate "commitment" and distinguish themselves, they may also reject other overconforming behaviors that do not align with the perceived integrity of the sport and the subculture. This view may be 
more in line with Heckert and Heckert's (2002) taxonomy of sport deviance that separates overconformity into socially acceptable (i.e., positive deviance) and socially unacceptable (i.e., rate-busing) behaviors rather than Hughes and Coakley's (1991) inclusive overconformity.

Moreover, whereas the reasons athletes engage in overconforming behaviors is not wellstudied, "Dale" did discuss the importance of overconforming in an effort to keep playing, as a response to self-esteem issues, and in an effort to prove his masculinity. For instance, on several occasions, and often when talking about his "peak," "Dale" discussed that winning was important because "winning means you get to stay on the court." When discussing the hazing/ bullying he experienced, "Dale" attributed being targeted by his attacker to his perception that he was "a scrawny kid... fairly shy... [and he] didn't really bother anybody." His efforts to prove his masculinity were most apparent when discussing his experience of fighting back against his bully and the resistance he displayed in the interview when asked about "less great times in sport." For example, he stated that "temper tantrums, pouting, running away" were embarrassing for him and that he did not want to share more information about instances in which he did those things. At one point, he even asked the researcher, "Why are you asking me to reveal skeletons that I don't care to?” By hiding instances where he behaved in a way he considered to be embarrassing, "Dale" was able to maintain his sense of masculinity and strength. His resistance indicated to the researcher the importance of anticipating and rolling with resistance. In future interviews, the researcher should re-frame several follow-up questions to ensure that they do not appear to be questions aimed at breaking down his masculinity, as it may be tied to the athletic identity in deeply-rooted ways.

Taken together, many of "Dale's" stories reflected the "act tough and give $110 \%$ " (Weinberg \& Gould, 2015, p. 462) attitude in sport that leads athletes to "play hurt or take undue 
risks" (Rotella \& Heyman, 1986 as cited in Weinberg \& Gould, 2015, p. 262). He reflected the sport norm of striving for distinction (Hughes \& Coakley, 1991) when he spoke about how special he felt that his coach was willing to let him live with him for two years to keep competing and how recognition from the media during his senior State meet made him feel. His consideration of staying with his coach is also reflective of the norm to sacrifice for the game (1991), given that he would have had to sacrifice living with his family for two years to do so. He ultimately chose to move away with his family, but nevertheless regarded that decision as the biggest struggle he had growing up. His willingness to compete his final match on a broken ankle and run laps after drinking milk to make weight demonstrated "Dale's" commitment to the norm that athletes endure pain and/or injury to continue to compete (1991). Finally, his goal to beat his rival in Districts, as well as his endurance in the sport, despite bullying, reflected "Dale's" belief that one cannot accept limits in the pursuit of excellence (1991). Dale's life story illustrated how athletes are driven to adhere to the sport norm in an effort to keep playing, which requires one continues to experience success in the form of winning, to gain social approval when self-esteem is low, as may have been the case given "Dale's" repeated references to being "scrawny" and "shy", and to prove their masculinity, as can be inferred by "Dale's" resistance during the interview. Thus, his story was roughly consistent with the work of Hughes and Coakley (1991), as well as Heckert and Heckert (2002), and provides an impetus for future research. 
APPENDIX H 


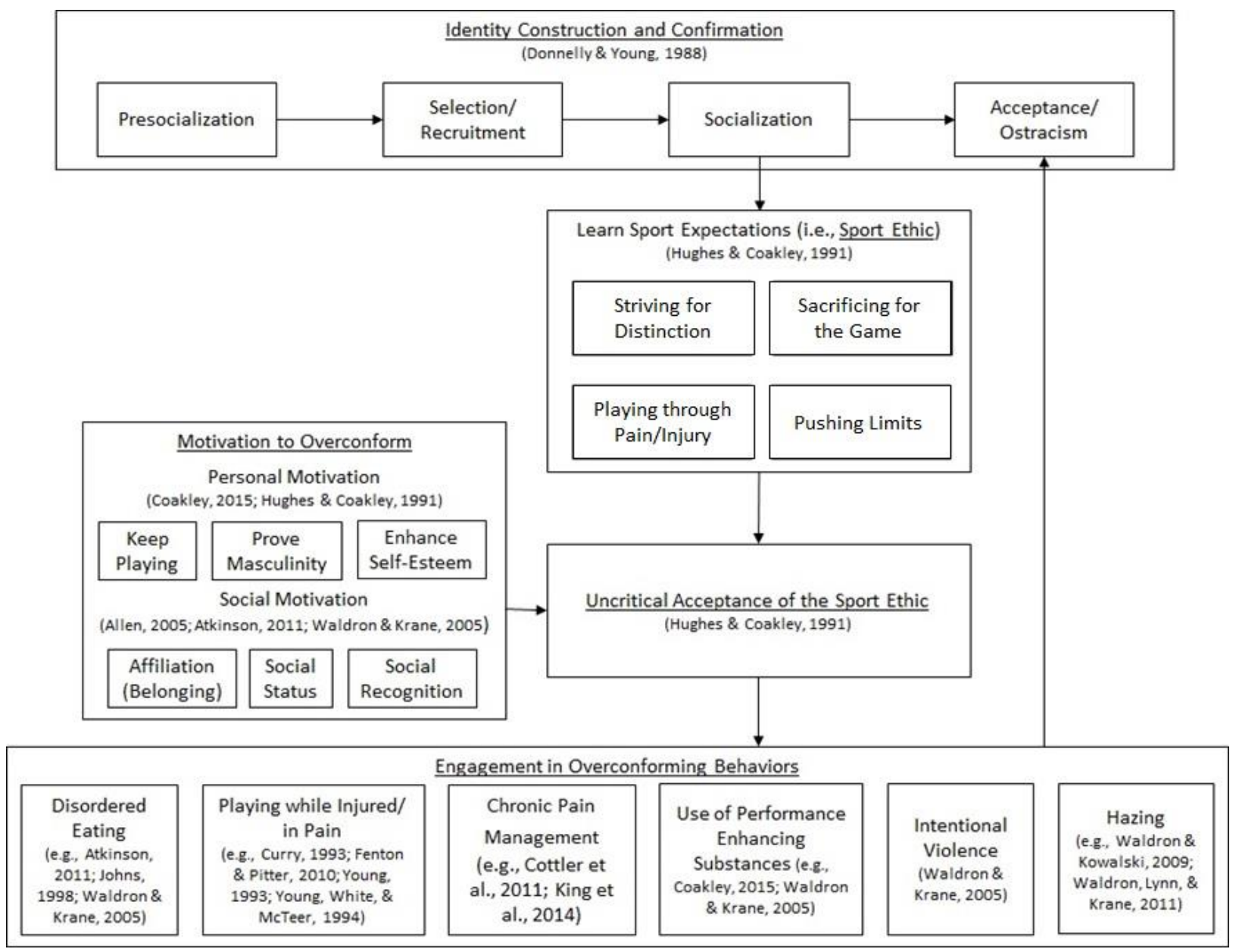

Figure 1. Conceptualization of Overconformity to the Sport Ethic in the Current

\section{Literature}


Percent Collegiate Athletes Engaged in Behaviors Associated with

Overconformity to the Sport Ethic

60

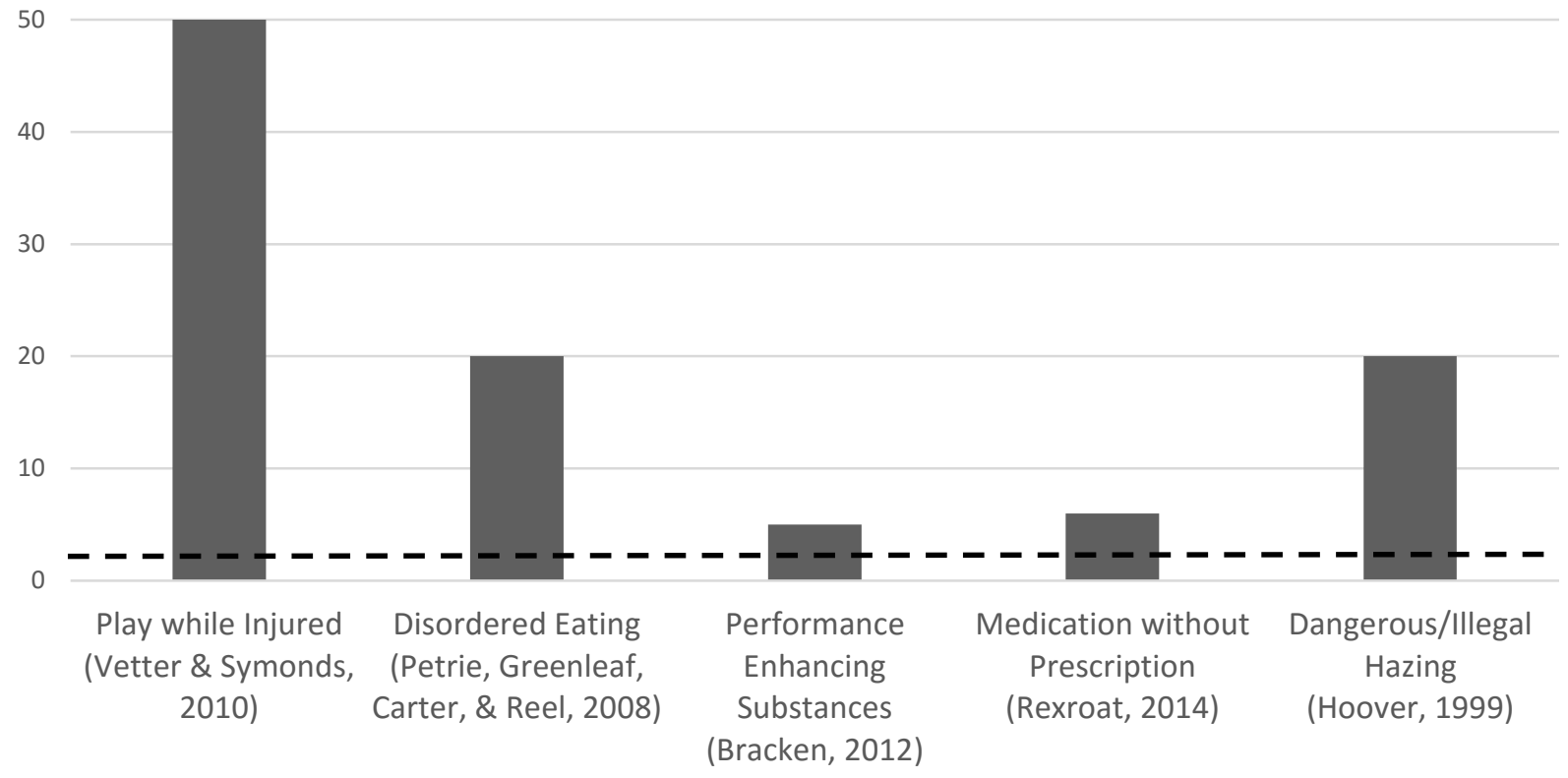

Figure 2. Prevalence Rates for Collegiate Athlete Behaviors Associated with Deviant Overconformity.

Note: Dashed line indicates expected prevalence rate $(2.5 \%)$ if behaviors were truly deviant according to the normal curve. 


\begin{tabular}{|c|c|c|c|c|c|c|}
\hline Purpose & Research Questions & $\begin{array}{l}\text { Theoretical } \\
\text { Frameworks }\end{array}$ & $\begin{array}{l}\text { Data } \\
\text { Collection } \\
\text { Methods }\end{array}$ & $\begin{array}{l}\text { Mode of } \\
\text { Reading } \\
\text { Narrative }\end{array}$ & $\begin{array}{l}\text { Coding } \\
\text { Employed }\end{array}$ & $\begin{array}{l}\text { Presentation of } \\
\text { Interpretations }\end{array}$ \\
\hline $\begin{array}{l}\text { Understand how } \\
\text { the "athlete" } \\
\text { identity is } \\
\text { developed, } \\
\text { ascribed } \\
\text { meaning, } \\
\text { performed. }\end{array}$ & $\begin{array}{l}\text { 2. What forms of } \\
\text { evidence do } \\
\text { athletes receive } \\
\text { from others that } \\
\text { identifies them } \\
\text { as athletes? } \\
\text { 3. In what ways do } \\
\text { athletes derive } \\
\text { personal meaning } \\
\text { from the "athlete" } \\
\text { identity? } \\
\text { 4. How do athletes } \\
\text { meet personal and } \\
\text { social expectations } \\
\text { of the "athlete" } \\
\text { identity in the } \\
\text { sport subculture? }\end{array}$ & $\begin{array}{c}\text { Identity } \\
\text { Construction and } \\
\text { Confirmation in } \\
\text { Sport } \\
\text { (Donnelly \& Young, 1988) } \\
\text { Overconformity to } \\
\text { the Sport Ethic } \\
\text { (Hughes \& Coakley, 1991) } \\
\text { Social Approval } \\
\text { Motivation } \\
\text { Orientation } \\
\text { (Waldron \& Krane, 2005) }\end{array}$ & $\begin{array}{l}\text { Round 1: } \\
\text { Life Story } \\
\text { Interviews } \\
\text { AIMS } \\
\text { SMOSS } \\
\text { Round 2: } \\
\text { Semi- } \\
\text { Structured } \\
\text { Interviews } \\
\text { Written } \\
\text { Expression }\end{array}$ & $\begin{array}{c}\text { Content- } \\
\text { holistic } \\
\text { (RQ2, RQ3, } \\
\text { RQ4) }\end{array}$ & $\begin{array}{c}\text { Narrative } \\
\text { (RQ2, RQ3, } \\
\text { RQ4) }\end{array}$ & $\begin{array}{c}\text { Creative } \\
\text { Nonfiction } \\
\text { (RQ2, RQ3, } \\
\text { RQ4) }\end{array}$ \\
\hline
\end{tabular}

Figure 3. Study Design Flow Chart 


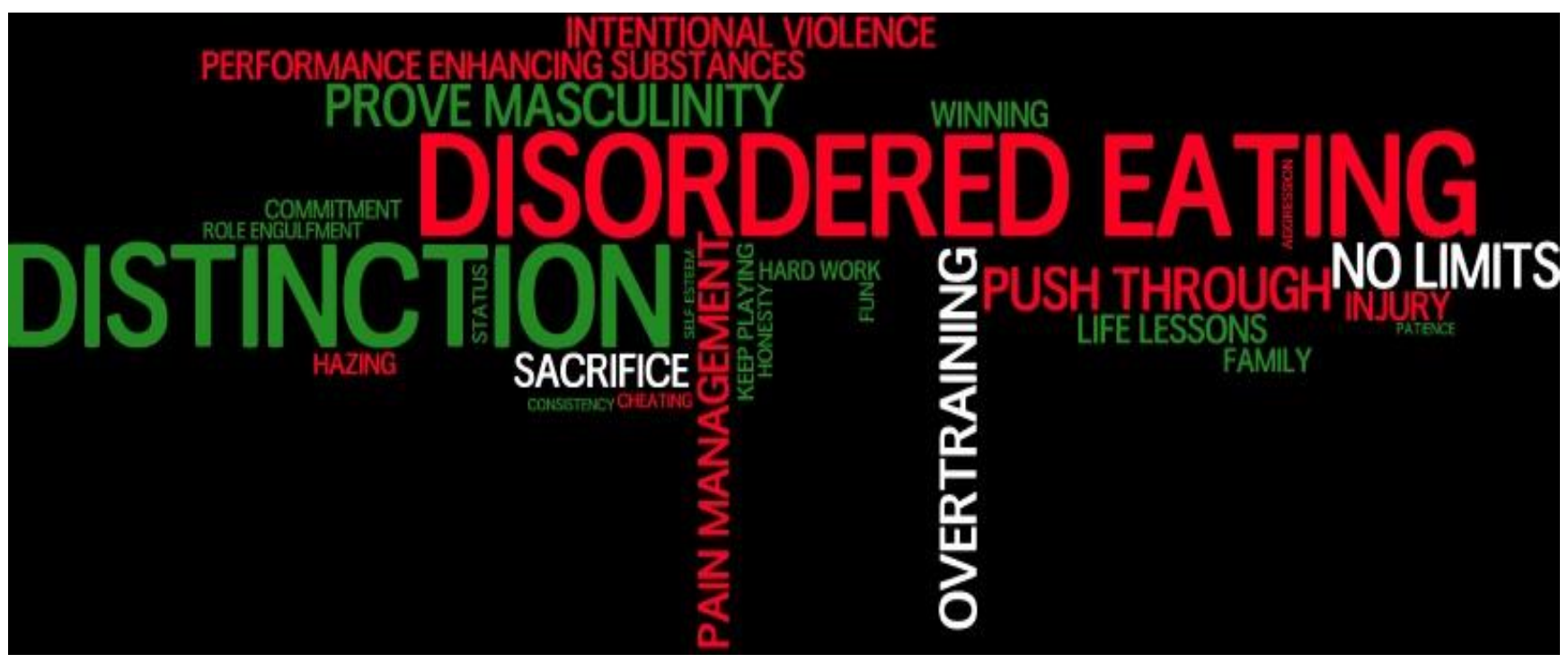

Figure 4. Athlete Identity Word Cloud 


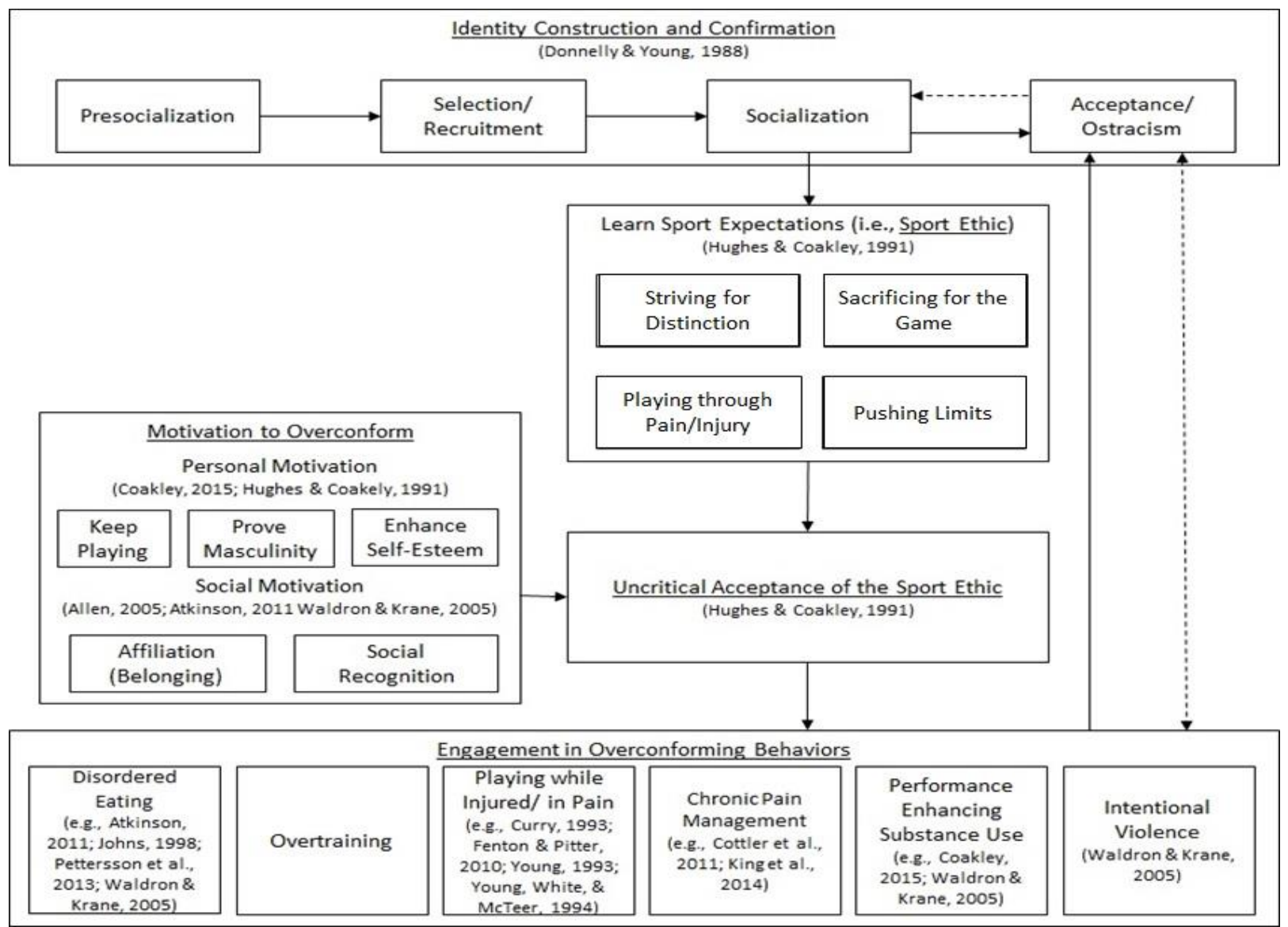

Figure 5. Overconforming Process for the Present Sample 


\begin{tabular}{|c|c|c|c|}
\hline & & \multicolumn{2}{|c|}{$\underline{\text { Normative Expectations }}$} \\
\hline & & Underconformity & Overconformity \\
\hline 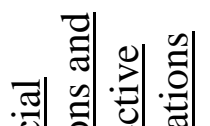 & Negative Evaluations & Negative Deviance & Rate-busting \\
\hline 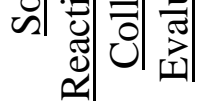 & Positive Evaluations & Deviance Admiration & Positive Deviance \\
\hline
\end{tabular}

Figure 6. Heckert and Heckert's (2002) Deviance typology. 


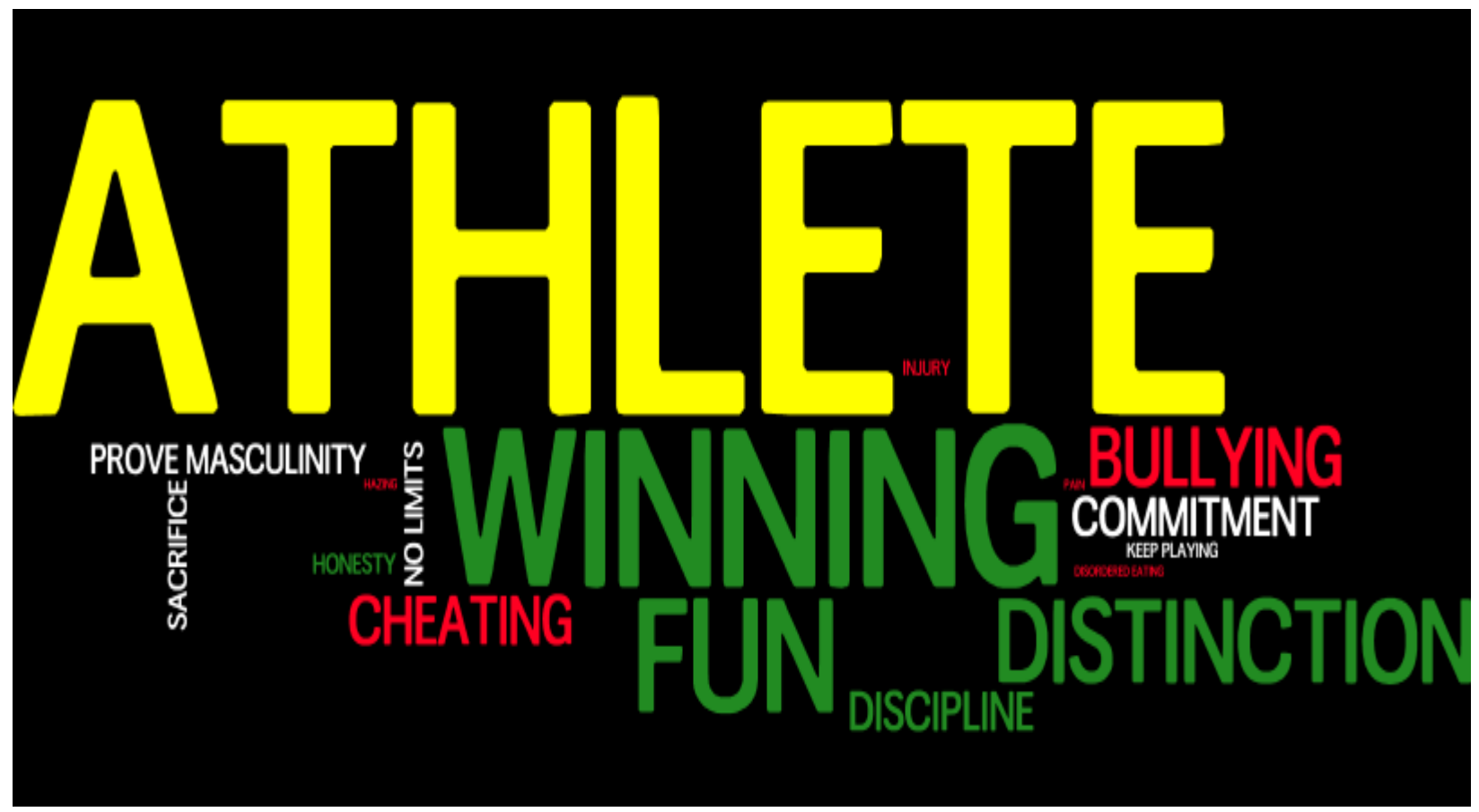

Figure 7. Word cloud created using words descriptive of the athlete experience, supplied by pilot interviewee. Note: Word cloud created using Wordle (Feinberg, 2013). 


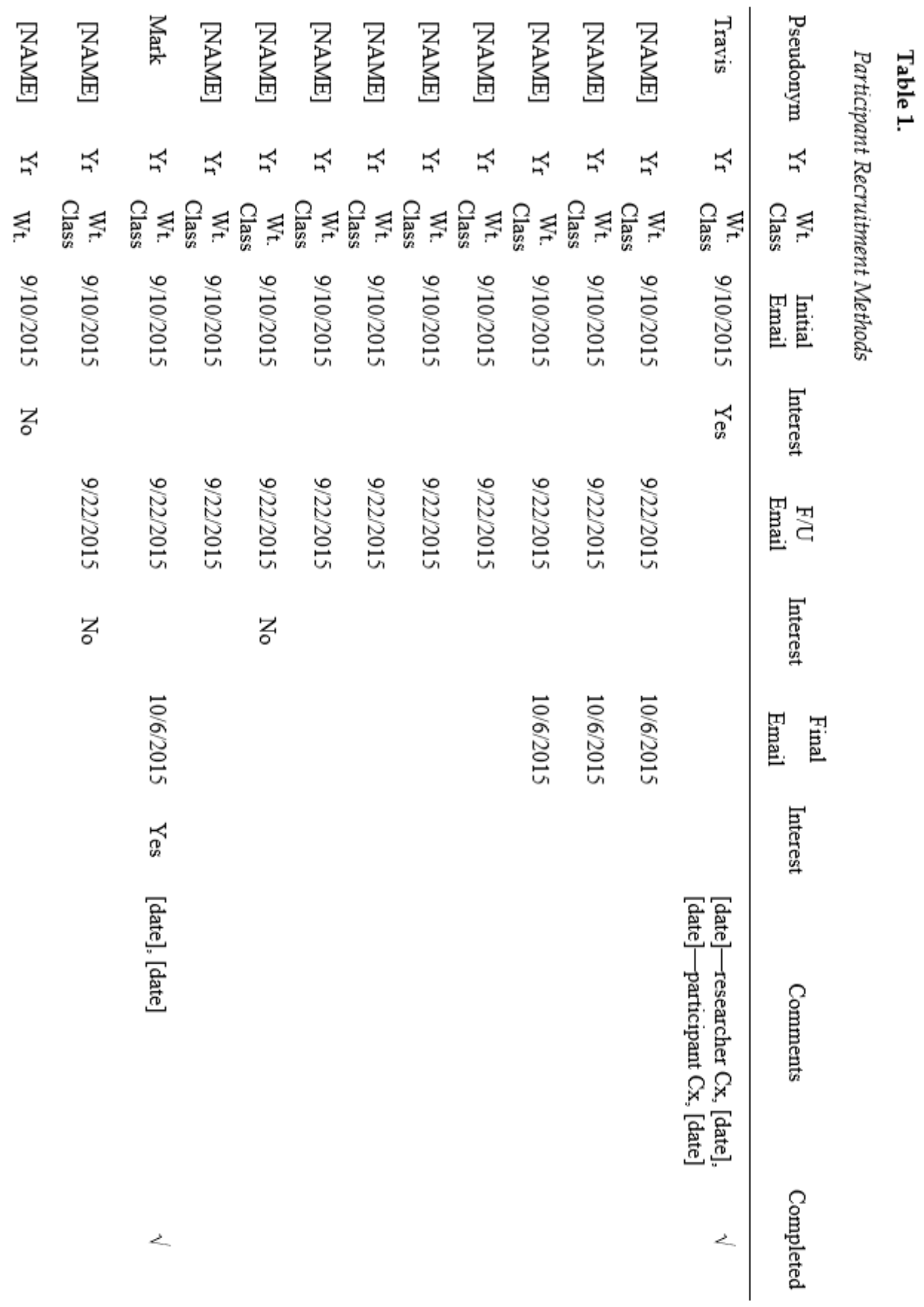




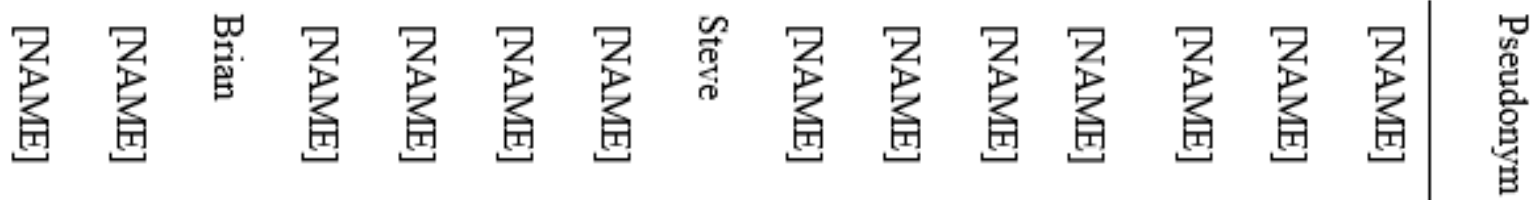

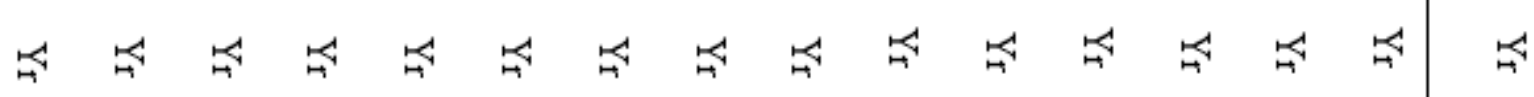

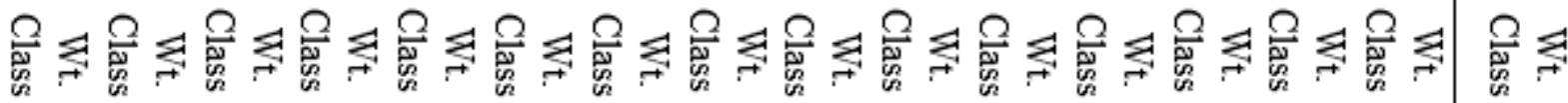

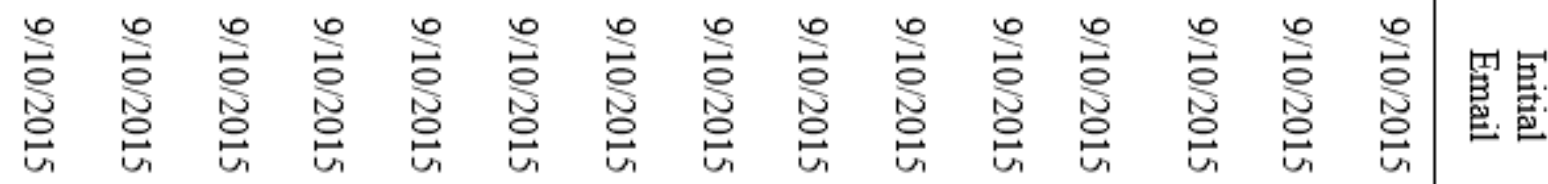

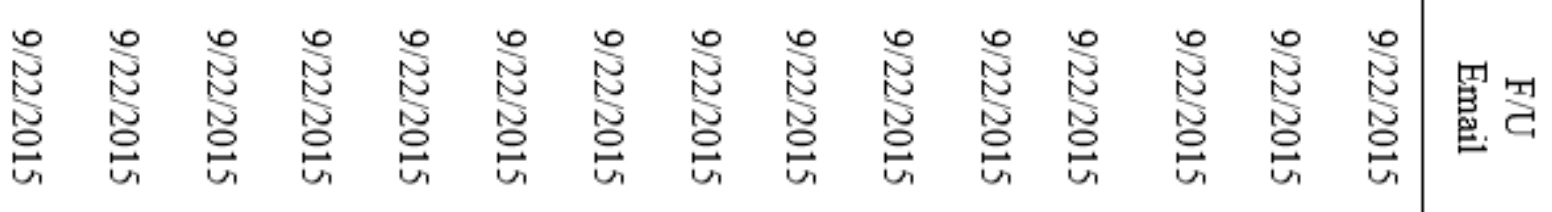

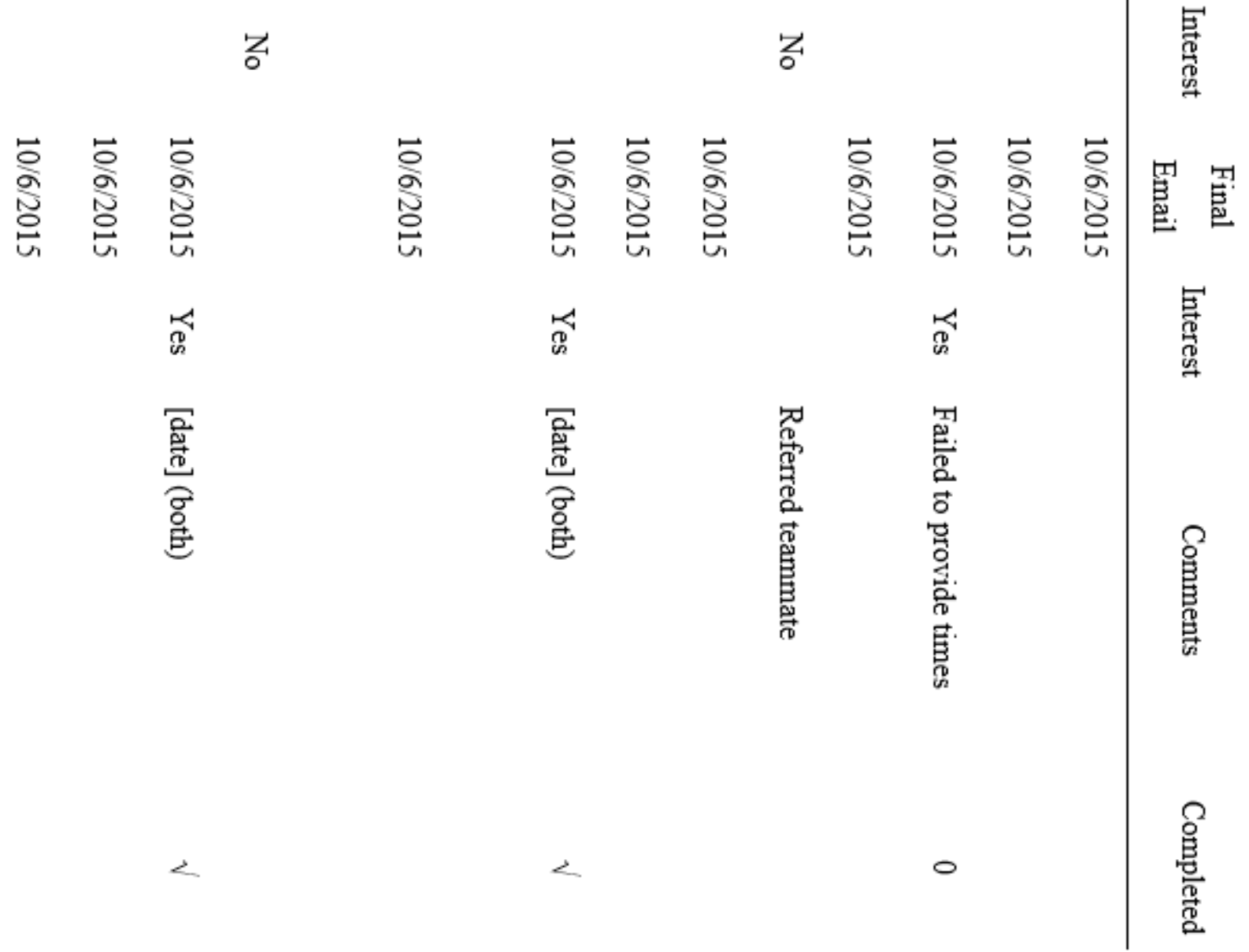




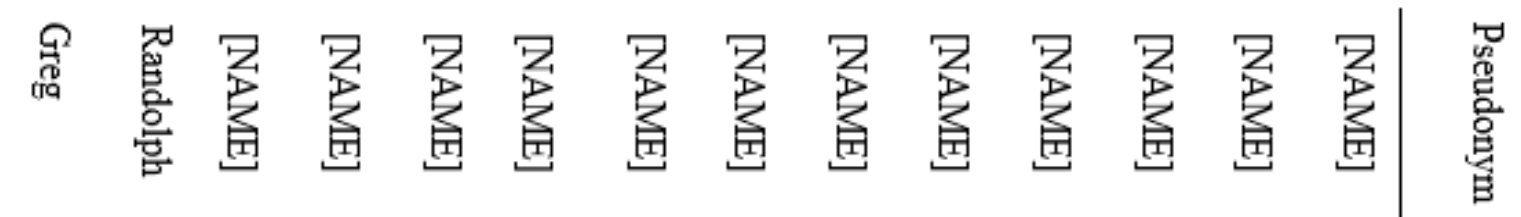

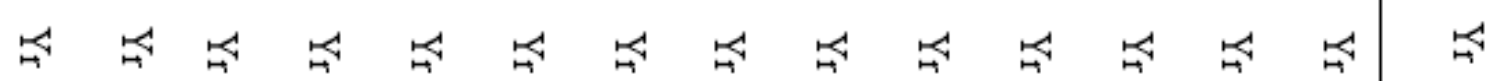

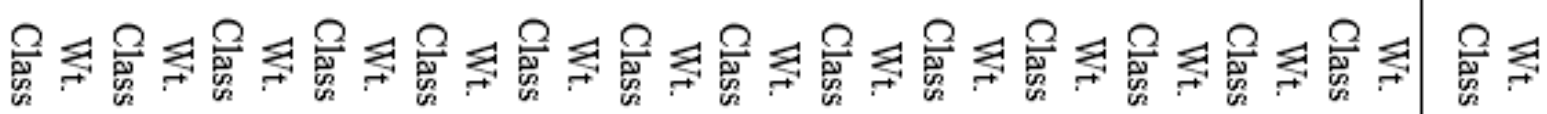

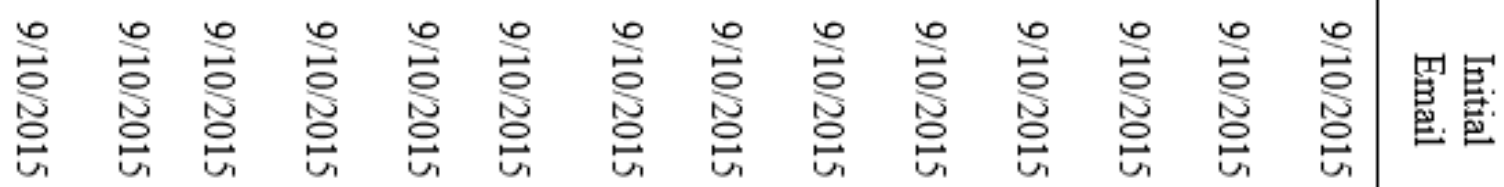

$$
\begin{aligned}
& \text { 萬 }
\end{aligned}
$$

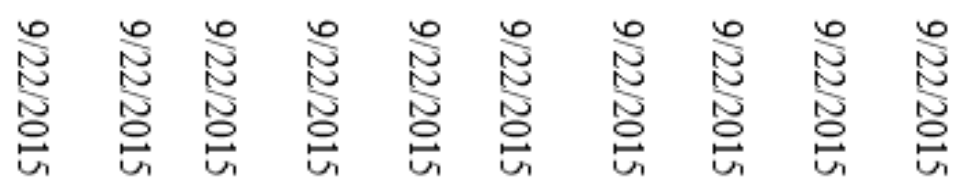

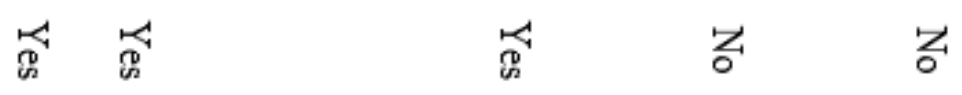

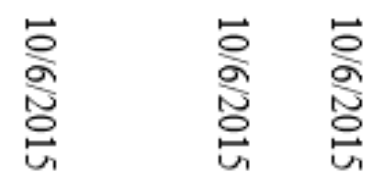

$$
\begin{aligned}
& \stackrel{\mathbb{R}}{\mathbb{R}}
\end{aligned}
$$

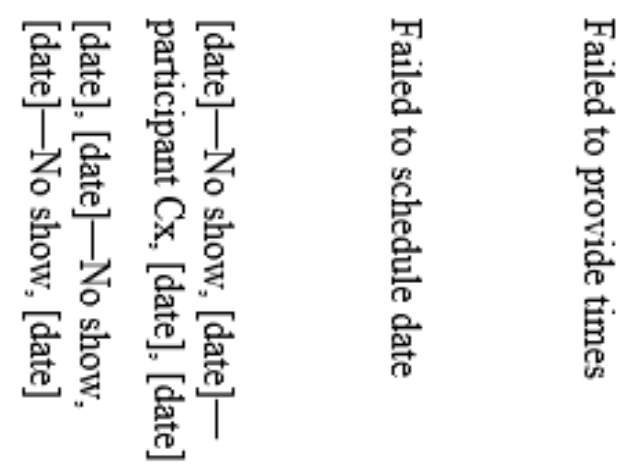

$$
\begin{aligned}
& <200
\end{aligned}
$$

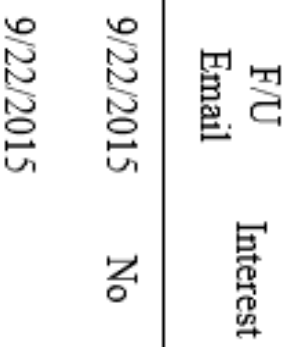

$$
\begin{aligned}
& \text { 四 团 }
\end{aligned}
$$

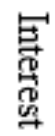

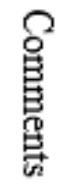

$$
\begin{aligned}
& \text { 葛 }
\end{aligned}
$$




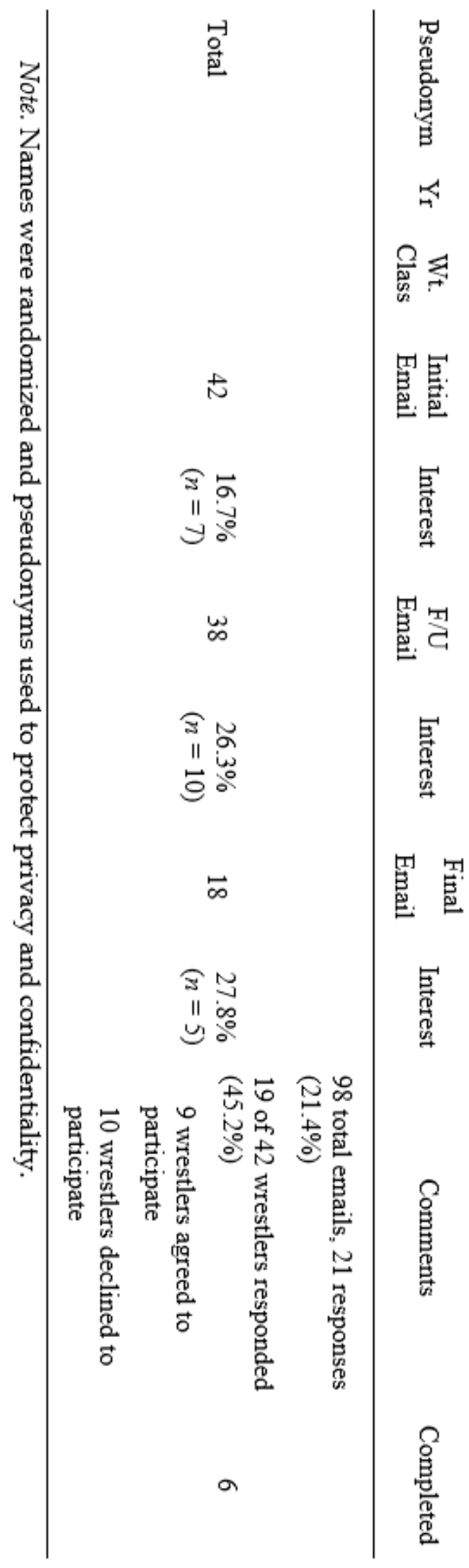




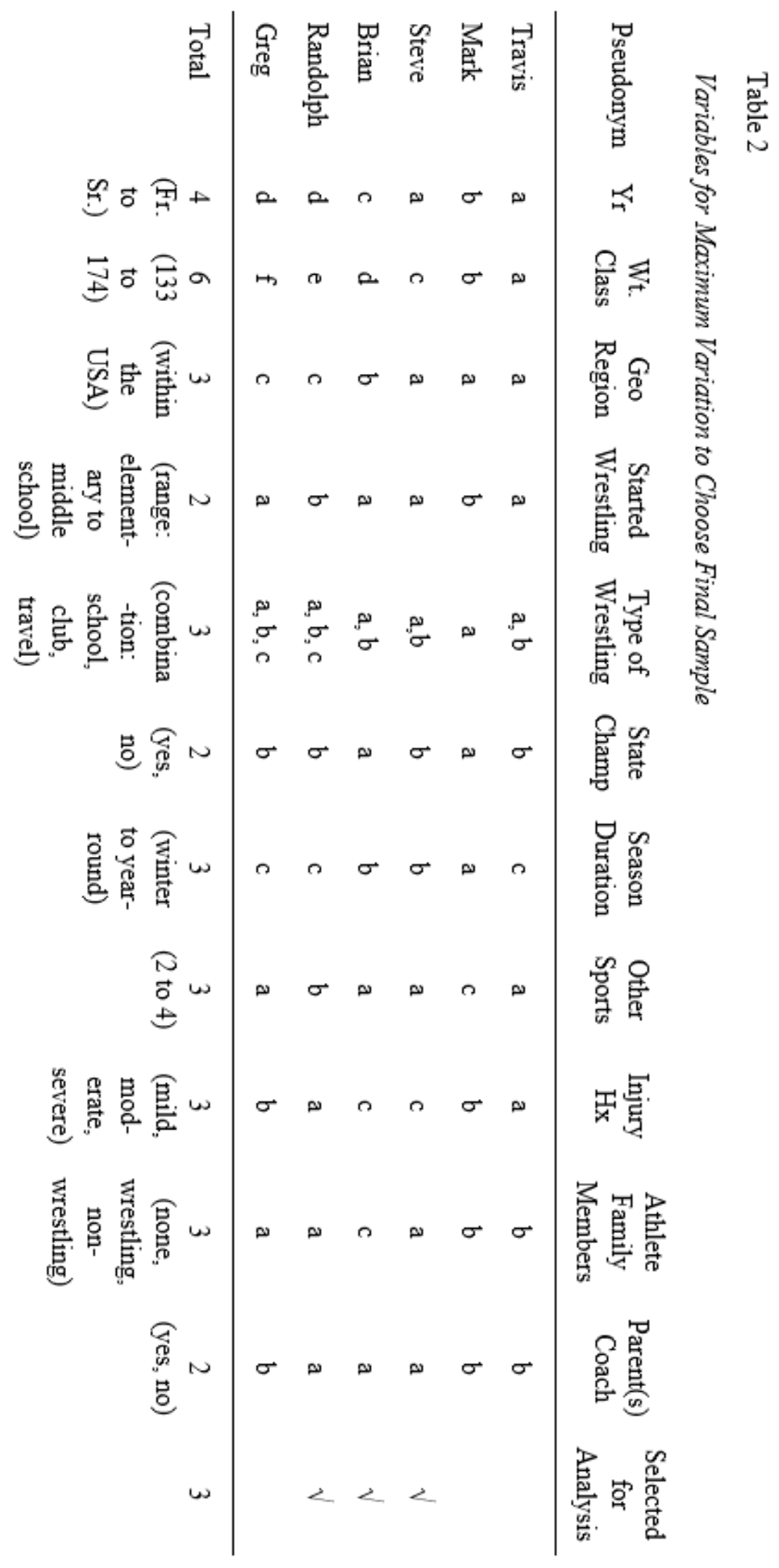


APPENDIX I 


\section{Analytic Memos}

August 20, 2014

\section{RESEARCHER LIFE STORY (ABBREVIATED)}

1) Peak

The day I got a thesis chair

The day I completed my data analysis for my thesis and found significant results

The day my thesis was returned revise and resubmit

The day I presented my SEC study results

2) Nadir

The day I was told I couldn't do a thesis because the advisor I was admitted under moved departments and I no longer had an advisor

The day my first manuscript submission (on my work with a SWAT team) was rejected from an applied journal for a lack of quantitative data

The day my revise and resubmit was rejected due to missing content (that I was told to remove). I was devastated

\section{3) Earliest Memory}

In my undergrad sport and philosophy class, we were doing a research project and I had no idea what to do!

4) Childhood Memory

I was always an observer

I have always loved to figure out how things work (like my brother's TMNT toys)

5) Future

I want to change lives with what I research.

I want to be a research guru. I was to be the go-to for qual/quant/mixed methods.

6) Turning Point

When I got into my lit review for my thesis and realized I actually loved research (the investigating, the writing, the measuring, the reporting - still working on loving the disseminating). I found that the more studies I read, the more passionate I became about the field. That, of course, also meant that I was bombarded by ideas so my chair finally told me that I couldn't save the world with a thesis. At first, I was crushed and incensed that the system was set up do encourage "just getting done." However, I've come to realize that if you never finish a project, you'll never share what you've found, and that helps no one. 
Thesis proposal-I killed it! It made me realize that I could actually do this.

August 27, 2014

\section{MY EPISTEMOLOGICAL STANCE}

I'm a post-positivist/constructionist. That is to say, perhaps, that I am a critical realist. The truth is out there, but we'll never truly understand the truth. We are all blind men with our hands on a different part of an elephant (the truth) and although we can describe our parts (the feet, the trunk, the tail) with details from our senses (some stronger than others), we cannot know what the whole truth looks like. Therefore, our understanding of the truth is based on our perception of it. When we investigate phenomena, we are asking different blind men to explain their experiences with the elephant, which they describe based on their experiences with other parts of the elephant and previous animals they've encountered.

My beliefs about understanding are that there is, in fact, one reality (no matrix here). However, the very act of perceiving that reality alters its meaning. We cannot remove experience from the knower any more than we can create a perfectly exact set of experiences between knowers (even identical twins must have some unique experiences; e.g., birth). Therefore, our understanding of reality is best-served by investigating both our experience with the phenomenon and the being/phenomenon itself, with the understanding that the knowledge we gain from that study is inherently imperfect (because no two beings or phenomena are exactly the same).

My position is in contrast with my old advisor (thesis chair) who believed that one could know the Truth as long as one used the right methods. Therefore, "failures" were the result of poorly designed studies.

October 8, 2014

\section{WHAT DOES MY WORK CHALLENGE?}

By deconstructing the institution of sport, and the resulting notion of "real athlete," we may, one day, challenge the belief that, in order to succeed in sport, to be recognized as a "member of the elite fraternity of athletics" (Hughes \& Coakley, 1991), one must betray one's body, must separate from the one-ness of self, in order to command the machine to do the work dictated by a society that demands the sacrifice of blood, sweat, tears, and physical well-being for the prize of the athletic identity. One day, hopefully, one athlete will challenge the idea of "real" athlete and pave the way for other athletes to question the heralded "uncritical acceptance of the sport ethic" and reject the sacrifice mandated by heroic archetypes long honored in the world of sport that have ruined many careers, bodies, and lives of sport participants, young and old. 
October 14, 2014

\section{WHAT ARE MY RESEARCH/INTERVIEW QUESTIONS?}

1) How is athlete defined?

a. What is your image of athlete?

b. Tell me about your story/life in sport.

2) How are expectations communicated?

a. Tell me about how you learned what it takes to be an athlete.

3) What does it mean to be an athlete?

a. What is/was your best/worst experience in sport?

4) What will athletes do to be identified as athlete?

a. Tell me about how you became an athlete. What did you do? How did you know you were an athlete?

b. If everything went perfectly, who would you be in $\mathrm{X}$ years?

November 4, 2014

\section{POST-INTERVIEW REFLECTIONS}

It feels good to get the first interview out of the way. Somehow, I was not quite ready for the potential stigma associated with this process. That's not to say that I wasn't expecting some resistance, I am asking some pretty invasive questions about several behaviors that tend to be closeted. And I did experience similar resistance with my thesis on disordered eating in female collegiate athletes, so I prepared for stigma about the behaviors I was examining, not about the research process, itself. In my introduction, I make a note to the participant that the life story interview is for research purposes, not a method of therapy, but I was not well-prepared for his response. It made me wonder if, by including that information into my introduction, I was actually planting the seed that the process might be therapeutic (see Theory of Ironic Processes). I'm not sure, I'll have to think more about this.

In terms of how the interview, itself, went, it was an interesting experience. My participant resisted me on several occasions and I think I handled it better sometimes than others. For instance, he was really interested in my opinion on one issue (I'll have to refresh my memory after I type this puppy up) and I tried to divert him. How can I expect my participants to tell me so much about themselves if I am unwilling to share anything about myself? On the other hand, this is for research and, although we are co-constructing the narrative, I don't want to add something that isn't there. But, then again, what if it is there and my resistance increases the participant's resistance not to share so I don't get anything? What to do...

I also need to read the transcript to check out when I led my participant. I didn't feel like I did 
this much, but I did hold on to a few pieces of information from him and ask him more about it. I'll have to talk to Malayna to make sure I'm not leading him.

November 13, 2014

\section{TRANSCRIPTION COMPLETE: REFLECTIONS}

Well, finishing the transcription is nice. It sure made me think a lot. I really felt like I was there with him during the interview, but there is a lot I missed. I hope this comes with time.

It was also good to be able to take a closer look at the participant's resistance and my role in creating and/or resolving it. For instance, he didn't want to talk about his lows, and I told him he didn't have to, but that I was curious about where his hesitation came from. As much as this was, clinically, the right thing to do, and he did open me, I think it made him distrustful of future attempts to do similar things. I'll have to watch for that counseling/consulting side of me in future interviews and keep reminding myself that this is for research, not therapy!

In another instance, he asked me my opinion about something, and I missed a huge opportunity to build rapport. I allowed the postpositivist side of me, the one that told me not to answer him, to outweigh the constructionist side of me that said it was fine. After all, if stories are coconstructed, I'll have to learn to be open to the process. I'll also have to remind myself that, even if the postpositivist in me says that not answering was the right thing to do, because stories are co-constructed, my refusal to answer likely did influence the rest of our interaction. I'm still not any closer to resolving my post-interview reflection about how I handled resistance, but at least I can look back and read it.

I've been really hard on myself over this past week about how I handled the participant's resistance, but at least I found one moment where I think I might have actually done a pretty good job. At one point he explained that he thought the point of an interview was that he was supposed to answer questions and I was supposed to analyze them. My response was

Well, that's the interesting thing about life story interviews is that some of the things that we talk about, you don't think about on a daily basis. And the interesting thing about life story interviews is that it's not just that you're answering questions, but you make very deliberate choices, even if their subconscious on some level, about what stories you are going to share and those stories say something about you because if you were someone else, you would probably choose different stories to share. So if you look back over the stories you shared, the memories you thought about that you didn't want to share, the values you expressed, how you thought you got to those, is there any kind of theme that runs through it? (553-560)

I think this was a good response because I was able to deflect and refocus him, while still getting him to buy in. He seemed more relaxed after this point, and more open, so I wonder had I spent 
more time talking about this up front, would it have improved the first half of the interview?

December 3, 2014

\section{IN-CLASS REFLECTION: MYSELF AS A RESEARCHER}

1) End of semester

By the end of the semester, I hope to see myself as a productive researcher. In some ways, I feel as though I've been quite productive (prepared two manuscripts for submission), but in other ways, I am disappointed with myself. I have always been highly organized, productive, and dependable. However, since having my son, I feel like a totally different person. In some ways, I love the person I am-I spend more time with my husband, am more present-minded, especially when I am with my son, which erases my stress about life when we engage, and I don't sweat the small stuff because I am much more flexible than I ever thought possible. However, as I get to know the new me, I have become aware that I feel like a part of me is missing - I mourn the loss of Ashley, the professional. Even though he is ten months old, and I am a mother, I am not sure that part of my identity is solidified, making me painfully aware that I am not the productive, dependable superwoman professional I used to be. I hope to regain the feeling of who I was, at least in part, by submitting my manuscripts, distributing my proposal, and giving myself a much-needed long weekend.

2) End of dissertation, five years later, end of career In the coming months, years, and decades, I hope to establish my new identity. I hope I can come to terms with the parts of myself I have lost and find a way to meet my needs in ways that are fulfilling to me, my family, and my field. I hope by the time I retire that I can make a lasting mark in this world through inspiring students, assisting colleagues, helping to change the sport subculture for the better, and further the field of sport and exercise psychology.

December 1-10, 2014

\section{ROUND 1 CODING REFLECTIONS}

First, I would be smart to put these in a word document instead of on the paper copy of the transcript. At the time, putting them on the transcript made much more sense, because I could see them right next to the transcript, so I had context whenever and wherever I needed it, but transferring them to a separate document now is a pain in the ass! I wonder how long it will take me to get this done... update pending... 


\section{Line Comment}

26 Interesting that, initially, sport ends for Dale when his formal participation, as a recognized athlete by other peer athletes is termination. Later, he discussed recreational sport experiences he had after he finished high school, but, initially, his "sport life" ends after his high school career ends

79 He seems to be struggling between what "chapters" society generally recognizes and the chapter he recognizes. On the other hand, he talks about how in childhood the games are supposed to be fun and "you're" not supposed to care about winning until later. On the other, though, he opens up that he "always wanted to win"

101 Recognition of accomplishments = high point

118 Interesting, later he talks about the importance of having fun, but he seems adamant that fun isn't enough - you have to win

120 Related to the sport ethic - you have to win to "stay on the court" and it is desirable to keep playing as long as possible ("if you want to keep playing, you gotta win")

151 Thinking of a high point was difficult, but he could remember "tons of low points"part of the athlete culture? E.g., with many reminders about what you're doing wrong with very little reinforcement about what you're doing right.

198 He mentions being scrawny twice. Once, it is in reference to peer bullying. This time, it is in reference to a coach taking interest in him, which, in turn, made him take interest in wrestling. It almost sounds like wrestling, itself, redeemed him from an identity of being "scrawny."

238 This almost sounds like it is central to the fun element in sport, even though fun is later represented as independent

249 Interesting that winning isn't positive, even though its mentioned several times

250 This sounds like central to his overall story, yet his is hesitant to give it the weight one might expect given that "they were some of the best times of my life"

274 I did not handle this resistance well!

328 Interesting that he chose this memory, reports it pleasantly, but then downplays it, as if he's not allowed to get excited about such a unique experience

333 Again, strong emotion displayed here, but then discounted because the sport is not deemed worthy of "feeling like a badass"

336 But earlier he states that sports are about winning and losing

361 If life stories are selective, why are the scenes he is remembering "stupid?"

374 The second narrative comes up again. He seems to have this tension about what makes a sport. On the one hand, it is something that requires a winner and a loser and is competitive. However, on the other hand, everything that is fun can be a sport

388 I missed a huge opportunity to build rapport here. The postpositivist voice in me said that it wasn't about me and I shouldn't answer, but the constructivist-interpretivist part of me said that was fine. After all, aren't stories co-constructed? And yet, I chickened 
out and refused to answer. Either way, whether I answered or not, would influence the rest of the interview; I just wish I would have chosen to engage more fully instead of standing at a difference

409 The coach wasn't good, so it may as well not exist

413 This seems to be especially salient because of the act of caring, as well as how it made D feel

432 Aha moment

$450 \quad$ Aha moment

458 So when the identity has to change, hold onto something else?

479 Again, the narrative tension between sport as a means for fun and sport as something sacred and competitive that should be honored and preserved. Here, he implies that the goal of sport is not fun, so cheating is not tolerated, but cheating is okay if the aim is to have fun (which sport is not in this instance)

483 Earlier, he said that cheating is blurry (474). Here, it seems clear-cut

508 After the interview, he asked me why I pressed him about cheating. I explained that some people view it differently (e.g., taking NSAIDs to compete on a broken ankle or purging right before a match might be just part of the game, but getting a lidocaine shot to block the pain or intentionally wrestling in an easier weight class for points might be cheating). His response was that, had I said that in the interview, his response would have been vastly different. HOW MUCH OF THAT IS LEADING THE PARTICIPANT AND HOW MUCH OF THAT IS AN ACCEPTABLE PART OF THE PROCESS?

513 Interesting that he started with "its blurry"

525 More tension between fun and... winning? I get the feeling that you have to choose between the two

553 I have to admit, I was impressed with myself here. He gave me a lot of resistance and I feel like I was able to deflect it and refocus him, getting more buy in pretty well

570 I'm not sure my bias could be any more obvious with this question!

n/a Semi-structured questions: At the time of the interview, I had not solidified my semistructured interview questions. Therefore, what follows is a product of our interaction together and the questions that came from listening to Dale's story. Interesting, BTW, that he was much more invested and descriptive during this portion. I wonder why?

592 He keeps saying sport, but it sounds more like he is describing athlete

640 Second time mentioning scrawny

651 Demonstrates how you can take it too far in an overconforming direction

683 I think he means coach here, not athletes

707 So we have more mixed messages. On the one hand, the answer "should" be conventional sports, and there's no place for participation trophies, but on the other hand, anybody can call themselves an athlete in their own way 
February 5, 2015

\section{Update: MALAYNA'S RESPONSE TO ME LEADING THE PARTICIPANT}

As open-ended as qualitative analysis is, the reality is that all researchers have to work with a purpose, or else it really may not be rigorous research. So, I shouldn't put words in my participant's mouth, but if I ask him to expand on something he brought up, perhaps I'm not really leading him, it was he who brought it up. On the other hand, by grabbing onto something and asking more information about it, am I not tipping my hand about what I'm looking for? Won't this bias my participant somewhat? I'm sure that I am, but how are you ever supposed to get in depth information about your research purpose if you don't explore the opportunities that come up?

February 9, 2015

\section{RESEARCH TEAM MEETING \#1}

So I've been nervous about putting together a research team (thanks to Malayna's Qualitative Data Analysis class), but I think it'll be good. I kept thinking I was going to have to explain too much because my research is founded in existing theory (unlike most students' data), but my team seems to get it. I even found out that not only is one member a pretty big deal in the formerathlete-turned-coach world, but my other, an expert(ish) in life story interviewing was a former national athlete. This should make for some interesting meetings. I look forward to our next one.

February 16, 2015

\section{RESEARCH TEAM MEETING \#2}

In some ways, this meeting was really useful, and in others, not so much. One of my group members did a great, thorough job analyzing my data, the other took more of a broad strokes approach. The latter was easier to follow in terms of the big picture, but I'm not sure I can qualify that as a contribution to interrater reliability. Of course, there's a lot of conversation in qualitative literature right now that questions the need for interrater reliability anyway. The former was great in terms of checking specific portions of the transcript, especially where I was worried that my biases were coloring my interpretations (whew! We were on the same page), but I found myself quite lost at times. She sometimes took me on tangents that I had a hard time following. At least she's sending me an excel file with her codes cleaned up, so I can look at those again during round 2 coding. 
Outside of coding, we all had a great conversation about choosing participants. It was interesting to see how the researcher with the experience in life story thought my pilot didn't yield much information (which is probably a function of the length and depth of many of her interviews and the different types of information we each focused on), whereas the researcher with more experience in many levels of elite sport thought my data was pretty rich. Our conversation gave me a lot to think about. Originally, I had not considered screening my participants before I interviewed them (I figured I should be grateful to get them since my population isn't very large), but maybe I need to in order to build some rapport up front (at least that's what my research team member recommended). Also, I had not thought I should oversample to make sure I get the depth of data I need, but maybe I need to consider it.

We also talked a bit about how much of my study's purpose I'll reveal to my participants. Of course, I don't want to lie to them, but if I tell them I am interested in knowing how/why they engage in disordered eating, playing while injured, hazing, performance enhancing substance use, and intentionally trying to hurt their opponents, I'll get doors slammed in my face. I need to find a way to be truthful but not too detailed. Perhaps I tell them I want to know how they learned what sport/athlete is, what you have to do to be a member, and what is acceptable/unacceptable as a member?

THE ROLE OF DEFENSE IN LIFE STORIES (McAdams)

The pilot participant keeps the bad part of sport under lock and key. Why is he protecting it? Why is winning the only acceptable thing to talk about?

February 9-23, 2015

\section{ROUND 2 CODING REFLECTIONS}

So, after the first time I wrote my analytic notes in the transcript, I told myself I needed to be better about getting them into a formal document the next round. Typical me, I did not do that, so here I am, compiling them all at once. This is not rigorous research practice-I seriously need to fix this for round 3 !

\section{Line Comment}

24 "Sport is life." What does this mean? What does it say about his socialization and other experiences?

55-59 He seems really stuck on what to call things moreso than the story itself

67-69 Fun is coming up a lot as the reason for playing sport

79-83 So there is some major transition - seeking some normalization

81 Did he mean elementary here?

100-103 Comparison, outcome of no limits? Maybe reading too much into this 
117-131 Just participating isn't good enough

123-124 I'm directing him here. Pull back?

127-128 You "can't" have fun... distinction?

130 Sport is not worthwhile if we don't keep score - where did this belief come from? Presocialization? Socialiazation?

133-136 Losing teaches the value of winning

$134 \quad$ Participation trophies $\rightarrow$ decreased excitement?

132-136 Theory: overconform to stay in sport b/c sport is exciting

144 No awareness of socialization process

150 Easier to think of lows than highs

$158 \quad$ Leading again?

179-181 Competing interests, logistical limitations $\rightarrow$ selection/recruitment

201 It is important that coach cared about more than just winning, but earlier stated that winning is the most important thing-paradox?

209 "not that he didn't care" but "he didn't give a shit"

211 Earlier, participation trophies are worthless, compared to winning, but when coach focuses on winning athletes, he's wrong

234 Sport is a site for socialization, even as a spectator?

249 Paradox? Sport value = learning or fun (which are not the same thing). What happened to the value of winning/competition?

258-264 Friends = fun, causing mischief $=$ fun, inactivity = boring. Must be active to have fun?

279 In sport, you have to be strong, cannot display weakness

284 Embarrassing reactions - sounds like hegemonic masculinity speaking

298-307 Organized physical activity = fun. In organized sport, it is all about winning, which is also fun, but there is a different tone

319 Sport was over because he was no longer on an organized team

322 In adulthood, shit talking and being and asshole is fun

334 Doing well in intramurals isn't important as winning in organized sport

335 Unorganized sport - maybe even fun - is not important, is not worthy of interest

344 Reference to the need to have winners and losers because without competition, life is boring

354 Still failing to see that story choice is personal

362 Wrestling subculture: binge (on chocolate milk) and purge (by running laps to make weight) 
375 So fun can cause a high, too?

$381 \quad$ Sport $=$ what you do for fun

$416 \quad$ Special? Want to participate because feel special? Distinct?

Distinction achieved via winning, coach attention, media recognition

$444 \quad$ Decreased social support $\rightarrow$ decreased risk-taking

$447 \quad$ Cynical because coach wasn't good. Ties back to winning? Distinction?

456 Future: fun = time with son. So no transmission of hegemonic masculinity to son?

462 Sport purpose as age $=$ health

Fun $\rightarrow$ winning $\rightarrow$ staying healthy

478 Cheating is okay if fun is the goal, but not for winning something important (like sport) is

$486 \quad$ Cheating is black and white?

499 Race to 100 brings in the serious, winning piece

500 There are limits to pursuing the win — cheating is unacceptable

525 Sacrificing enjoyment to prove commitment?

$525 \quad$ Fun is not a value

529 Learn about sport from friends, coaches, family

$537 \quad$ No wonder cheating is a black and white issue

567 But conventional sport matters more? Remember flag football...

619 Tension between sport mattering and sport as insignificant

$640 \quad$ Scrawny $=$ bad, shy = bad. Blaming the victim?

$647 \quad$ Culture of bullying?

649-650 Hazing = acceptable until one crosses the line to asshole

683 Sacrifice deserves respect, even as staff

686 Continue to socialize the importance of sacrifice

$704 \quad$ Hard work $\rightarrow$ distinction as athlete (in conventional sport?)

711 "right" to refer to self as athlete (as long as you put in the work) — but participation trophies are not worthwhile?

March 25, 2015

\section{PILOT DATA WOES}

How on earth am I supposed to put my pilot data into my document?! Why don't they write a 
book for that? Or for any formula about how to write a qualitative dissertation, for that matter? I suppose I have Creswell's book, but that's ridiculously vague, so all I have is a basic outline and I'm not even sure it fits my study. I'm all for being creative, but this is a dissertation, not a canvas!

April 6, 2015

\section{FINAL CODING COMPLETE!}

After three rounds of coding, I finally have a beautiful excel file of my pilot data. I coded it by type of code (life story, provisional by theory/component, new) and I think it'll really help me to interpret it all. I had the brilliant idea that I was going to present my analysis as a word cloud to answer RQ1 and as a narrative for RQ's 2-4, but I'm not sure how to actually do this. I saw an example of using narrative to represent compiled results in Waldron, Lynn, \& Krane (2011) and thought it was particularly consumable and alluring, I just don't know how to actually do it, yet. Would it be wrong if I just started playing with it to see if it is worthwhile before I look for research support?

BTW, definition of insanity: "doing the same thing repeatedly, hoping for a different result" (citation needed-I've been in dissertation mode for FAR too long!). I went through two rounds of coding where I wrote all of my memos in the margins and had to transfer them to the computer later, which was a pain in the ass. When I listened to a classmate talk about it, I secretly wished I could honestly say I thought he was nuts for writing it down instead of typing it up, but I was doing the same thing. I know I prefer to grade papers in hard copy, which is probably why I like coding on paper, but I seriously have to change things for my actual participants or I'm going to waste more hours on end than I care to think of. I think a lot of my resistance, too, comes from the fact that I like to be able to look at the actual transcript for context of my memo without having to re-type it, so I suppose I'll have to find a system. After all, it worked for Mac. I just need to commit to the extra step up front so I can save time on the background.

April 9, 2015

\section{WORD CLOUD, IT IS}

I just spoke with Dr. Watson about my final coding and showed him an example word cloud. He seems on board. He suggested I change the colors to reflect positive (green) and negative (red) codes, which probably isn't a bad idea. I have no idea how to do that, but at least it is a fun part of the project! 
On a side note, I figured out how to leave brief notes on my phone if I'm not sitting in front of a computer, which is making it easier not to forget my thoughts between checking in.

April 17, 2015

\section{UGH! THIS PILOT DATA IS KILLING ME!}

I have been trying figure out how the hell to incorporate this pilot data into my manuscript for weeks to no avail. I've tried it in the intro and in the lit review; I've put it in the methods, too. It just seems to disrupt everything wherever I put it. I'm so taking a poll about what other people do/have done!

April 20, 2015

\section{SUPPORT FOR WORD CLOUD AND CREATIVE NONFICTION}

I'm not sure why I didn't think to look in Waldron, Lynn, \& Krane's (2011) work for their source on using narrative to present results, but that was a much better idea than aimlessly trying stuff. I just found Caulley (2008) and will use that to guide me.

I also found a source (McNaught \& Lam, 2010) that talks about the utility of word clouds to present qualitative data. They may be looking at literature and blogs instead of SEP interviews, but the same principle applies. I'm finally getting excited for this!

April 22, 2015

\section{SO I'VE AGREED TO STOP LOOKING FOR SOURCES}

Why am I so hesitant to just finish this proposal? Dr. Watson told me I'm not allowed to look for any more sources today, which, to be fair, he also told me to do back in March. Why am I feeling anxiety about it? If anything, I should be jumping for joy. After all, he's read my drafts and turned out a ton of great dissertations... I have to just learn to trust him.

April 23, 2015

I'M REMINDED OF THE PHD COMIC WHERE THE GRAD STUDENT HAS A LIGHTBULB MOMENT WHEN SHE'S WITH HER PROFESSOR

And then she walks out of the office and everything goes dark. I just met with Malayna again 
today. I've been so frustrated about how to incorporate my pilot data and nothing seemed to work. I think we've agreed just putting it in its own section in the Methods section. I'll do that today or tomorrow and work it out.

But now that I've made it back to my office a whole ten minutes later, I feel like I lost everything we talked about. I know there was something about the term "reflexivity." I know Dr. Watson hates it, and would prefer I use "reflection" but I am hesitant to stray from the accepted lingo in qualitative. Surely, I have to be making a bigger deal of this than it is... perhaps I should just talk to him about it.

I'm also fairly sure that we talked about a future study/line of research that got me totally jazzed about looking at what careers overconforming athletes choose (e.g., stricter boss, explicit expectations), which also means that I'd also have to look back at the role of the parents. While I remember that part quite clearly, I cannot remember the other things we talked about, which is stupid... I need to be better at writing it down.

Update: Malayna doesn't recommend I oversample. She says that if I don't get what I need in three participants, I can always look for more, but I don't necessarily need to plan it up front.

April 25, 2015

\section{FUTURE RESULTS/MANUSCRIPTS}

"Dale" had a very strong relationship with his first coach. I wonder if that relationship buffered some of his overconformity behaviors. That is, in my thesis (which should be published sometime this year), I found that the coach-athlete relationship was a mediating variable between athletes' perceptions of weight-related coach pressure and disordered eating behaviors in female collegiate athletes in endurance sports. It might be a good idea to compare my findings from this pilot with other participants to see if there are any discrepancies in the athleteperceived coach-athlete relationship and overconforming behaviors. If so, it could be a good area of future study in male athletes, as well.

I've also been thinking, today, as I've read and re-read these memos to make sure I've addressed everything before I turn this baby over to my committee and had another (brilliant?) idea: for a second manuscript, consider a methodological piece about this process. I even have a title: Rigor in Qualitative Analysis: Lessons Learned from a Graduate Student Who Did It All the Wrong Ways. Perhaps it would be useful for an SEP-specific journal since some has been written about narratives in SEP, but only a few on life-story research? Perhaps not, might be a moment of narcissism... who knows? 
April 26, 2015

\section{EXISTENTIAL CRISIS?}

As I read back over my proposal, review the literature on specific overconforming behaviors, and revisit my analytic memos on "Dale's" experience, I can't help but wonder if I really have justification for this study. That is, "Dale's" story reveals instances of overconforming behaviors (e.g., wrestling on a broken ankle, running laps right before a match to make weight) and instances of unrealized overconformity (e.g., nearly choosing to live away from his family for two years to consider wrestling at a distinguished program), but do those instances really have lasting negative health consequences? I've set up my argument that overconformity is important to study because it can lead to health issues later in life (e.g., disordered eating to make weight may have negative physical and mental side effects that influences one's relationship with one's weight and eating/exercise habits later in life, competing with chronic injury has the potential to create long-term disability, risking injury in the first place means placing one's body in a situation that could have disastrous effect for the body, taking performance enhancing steroids that are not regulated by the EPA can mean ingesting/injecting harmful substances into the body). However, if an athlete rarely engages in the extreme versions of these behaviors, is there really much risk? Are athletes who sometimes overconform but mostly "just" conform in the same boat? How can we quantify the psychological risk that expectations of adherence and reinforcement of uncritical acceptance of the sport ethic pose to the individual athlete who rarely or frequently commits? Should we be distinguishing, in the literature, between a lifestyle of overconformity and instances of overconforming behaviors? Is this an important point that needs to be acknowledged? Is it outside of the scope of this study and an area of future research or is it an important distinction that needs to be explores?

\section{April 27, 2015}

\section{FEELING A BIT MESSED UP}

I've just read back over my proposal. I was supposed to have it to Dr. Watson on Friday, but was still editing, so I asked to work on it Saturday. I was convinced I could finish it Saturday and give it to him to peruse on Sunday. By Sunday, I was still not done and starting to worry. Last night, having still not given him a copy, I found myself lying awake trying to figure out why I have been so concerned about needing an extension, and why I am obsessing over this proposal. After all, it won't be perfect-it can't be - and how am I supposed to make it better if I won't give it to my advisor and committee to read? I resolved myself to read over it one last time and then send it off today. After reading my researcher background again, I have two reactions: 1) sport really messed me up in a lot of ways. At least, my relationship with sport led to a few issues, but I am also reminded about Coakley's (2009) distinction that sport is a site for socialization, not the agent, so I really can't blame sport, I'm sure I would have found another 
way to mess myself up; and 2) I am falling into old patterns about needing to be the best, to be perfect, not to let others down (although I do understand that it is really more myself, not others I need to worry about) so I need to get myself back on track. I think tonight, after I get home, I'm going to take a long walk with the dogs and remind myself of all of the things I am good at. I think having a long talk with myself will help to steer the ship away from the jagged rocks awaiting me if I stay on this bearing.

\section{THE BIG PICTURE}

1) How does my work contribute to the field? Overconformity is understudied, but the consequences are potentially devastating. The researchers who do include it tend to do so for a very narrow set of behaviors. I hope to re-ignite the conversation. After all, how can we prevent/treat problem behaviors if we lack the empirical understanding of why they occur?

Also, a case has been made to use narrative and life story research in SEP, but it still has not caught on. I hope to inspire to inspire others to use these methods (plus word clouds and creative nonfiction as methods of representing the data) to understand phenomena in our field better and engage all readers (not just scholars) in new, meaningful ways.

2) How might my approach to data analysis support my contributions to the field? It is still pretty new, which makes it exciting. Plus, the product (word cloud and creative nonfiction) makes the work exciting.

3) How might my analysis move me into new conversations for fields of study? Refining theory, opening new ways of conceptualizing the issues. Bringing together research that is currently not in conversation with one another.

Challenging creative flexibility. Expanding my toolbox.

\section{CODING THOUGHTS}

Mac said something in class tonight about process coding. I wonder if that would be important/appropriate for understanding the process of socialization (and developing the athlete identity). I wonder if I do that as a separate round of coding or simultaneously with my provisional codes. Remember to look more into this...

April 29, 2015

\section{WELL, I THOUGHT I HAD A GOOD PROPOSAL...}

I've just heard back from Dr. Watson about my proposal. At first, I admit, I was overwhelmed- 
I think he saw my fear in the whites of my eyes. However, after working through his suggestions, I think my proposal is stronger (I don't know how I forgot to include limitations-rookie mistake!). That being said, I am still left with a few questions I don't know the answers to:

1) How do I thoroughly present/justify methods so new to the field? I am using concepts and rationales that are unfamiliar, which means I have to explain them in deeper detail, but that also takes up space (which I need for results and discussion/conclusions).

2) How do I balance being vague enough to allow myself to let the data talk without getting so vague I get too much information (i.e., Dr. Watson's question about what "too far" means in the semi-structured interview) with being specific enough to gather the information I need without being leading (i.e., Dr. Watson's concern about the written expression being leading)? The last thing I need is an Achilles heel before I even start this thing!!!

3) I keep coming back to something I thought about a week ago: is there a distinction between athletes who overconform occasionally and those who do so on a regular basis?

Do I need to know this ahead of time or is it something I can just look for in my data and come back to later?

4) In an effort to consolidate my study and keep it manageable, there is a lot out there I have not explored. For instance, when looking at athletic identity development, I looked solely at Donnelly and Young's (1998) work because that is what was used in Hughes and Coakley's (1991) original work which is at the foundation of my study. However, because I did that, I have not looked at the wider athletic identity literature for changes in how we conceptualize the athlete identity. Is this something I can come back to, to explain my results or do I have to address it up front? Also, I only looked at Hughes and Coakley's (1991) framework of deviance, but Heckert and Heckert (2002) offered one as well. Again, do I address that more extensively now or wait for the data to tell me what to do? From an inductive standpoint, I am inclined to do the latter, but from a traditionally post-positivist standpoint, this feels less rigorous. On the other hand, I am looking specifically at Hughes and Coakley's (1991) framework for a reason, so perhaps I've done enough work on the front end. I just don't know what to do.

I am slightly concerned I don't know the answers to these questions. Is it acceptable to just ask my committee what they want me to do? Is that a cop-out?

May 11, 2015

\section{THE EXISTENTIAL CRISIS IS BACK...}

So, after a few laps with Dr. Zizzi (awful day to wear jeans in the summer!), I'm trying not to freak out. He spoke a lot about considering how I will develop a funding line in this area, which made me question the feasibility of this line of research long term. Will it be relegated to pet 
projects or can I make a living out of it? Going into this project, I knew I had two options. The first was more fundable: look at exercise prescriptions for combat-related PTSD. The second was less fundable, but I was more passionate about: this project. It was flattering that Zizzi thought I could write a book about this stuff, but given that my goal is to reach a research-intensive university and become a tenured faculty, I'd be lying if I said I wasn't nervous. He told me not to make crazy changes to my project, but I can't help but wonder what I should do.

\section{BUT I'M FEELING REINVIGORATED...}

So, I wasn't much feeling up to doing my mock proposal today, but Dr. Watson made me do it anyway. I'm feeling more convinced than ever that I really am heading down the right path for me... I'll keep plugging away.

June 10, 2015

\section{I'M UP TOO LATE TO BE THINKING ABOUT MY DISSERTATION, BUT I'VE BEEN PUTTING A LOT OF THOUGHT INTO BEING MORE BOLD (AS MALAYNA RECOMMENDED).}

Here it goes...

In counseling, we ask our clients to illustrate, rather than narrate. Instead of asking a client to tell us what it's like, we ask them what it looks like. If a client says, "it's hard" or "you have to be strong" (both narrations), we ask him to pain us a picture. Again, we may ask, "what does it look like?" or "what's an example of when it was hard or you had to be strong" (both illustrations). Asking for illustrations provides context which helps us get into the client's world so we can better understand, and better help them. If research truly seeks to understand, why not employ the same principle? Illustrations deepen our empathy (we'll never truly understand what a client's experience is like, but we can sure relate to it if we have the context). Deepening our empathy makes us care about issues, which prompts action. Illustrations deepen our understanding of context, increasing our accuracy of associated issues; greater accuracy leads to greater predictability which leads to greater efficacy which means our action has a more positive effect. Why, then, would we settle for narrations rather than illustrations? Sure, it saves time... or does it? Perhaps putting the work in on the front end saves us time on the back end and results in more accurate research and more effective prevention and treatment. Thus, this study seeks to illustrate overconformity in collegiate wrestlers, in its varied forms. The result of one single definition is practically insignificant; what's more important here is the transferability of findings, examined deeply and accurately through illustration. The results of the present study are presented here as an illustration to prompt the reader to empathize and take meaningful 
action.

Of course, now that I've said all that, I've been thinking about Malayna's question about my presentation of results: "Why is creative nonfiction the best way to convey my findings?" I don't have the perfect answer to that because I'm still dealing with my own insecurities as a researcher-after all, I'm just a graduate student, not an established researcher to whom people care to listen on matters of research methodology. However, if I push myself for some rationale, here it is. Traditional qualitative researchers, present their findings as themes from grounded theory, phenomenology, and even narrative research, are illustrative. They use verbatim quotes to illustrate researcher interpretations of the data. In that way, they do a great job of illustrating points. So, if quantitative findings (at least in the case of survey data) provide a snapshot of the participant experience, traditional qualitative findings (presented as themes present in the data) become a comic strip (with or without the humor). Even this is limited in depth and richness of context, though, which are necessary to contribute to accurate empathy. Wouldn't it be nice, instead, to watch a video of the findings, to really see the participant experience? Sure, the full footage is too cumbersome for participants and consumers and, still, the researcher (the movie director) exerts bias by choosing what to film and what to cut and how to compile it for the viewer, but how much richer an experience is it to watch a movie than read a comic strip or glance at a Polaroid? Presenting qualitative results as a creative nonfiction places the researcher in the director seat, albeit with a superstar actor who still calls many of the shots. Being in the director role allows the researcher to create a compelling, important piece of work that takes the viewer deep within the actor's world to better make sense of the experience and connect to it emotionally. As mentioned previously, this emotional connection is a result of empathy and prompts action. Now, transferring the film to print is easier said than done. After all, research is consumed in journals, not on the big screen, and seeing the actor (participant) would be unethical. Not to mention, when the film is transferred to print, we lose the visual cues and nonverbal communication that connects us and deepens the story. However, if we can present a good script, we can recapture many of those cues and nonverbal. Creative nonfiction can be that script.

\section{AND NOW THAT I'M ON A RANT ABOUT METHODOLOGIES, LET'S JUST GET IT ALL OUT THERE...}

Here's my resistance to statistics... well, not statistics, per se. In fact, I love number crunching. However, I have a strong resistance to how statistics are often used. You see, I'm the outlier. When the doctor tells me to take a drug, I'm very careful to ask about side effects because I'm the one in ten thousand that will get the obscure, miserable rash from a routine vaccination or the epidural that doesn't work. That's right, I'm that one percent who gets forgotten because, statistically, I'm the minority. So, when I hear that something is effective for 95 percent of people or that a hypothesis was supported with 95 percent confidence, I think, "How great for the 
95 percent, but what about the other 5?" Perhaps that's why I'm interested in deviance, the five percent that the world forgot because one's odds of being the five percent is rare, so no need to waste the resources. As Kant said, sacrifice one for the fortune of many, right? But used this way, statistics is limiting. It says, "look here because this is the most bang for your buck," which is a great, much needed starting point, but when the remaining five percent are forgotten, there's a problem. This leads me to my stance on research: quantitative and qualitative methods should be iterative, not exclusive. Sure, some questions are better answered with certain methodologies, but when we start putting research questions into boxes based on whether they check the traditional quantitative or qualitative box, we limit our ability to understand the human experience. Whether the iteration starts with qualitative methods because we know something is happening but we don't know what it is and want to see it or we start with quantitative methods because we have some ideas but need to see if we're right, in my mind, the next step is always to use the other approach to fill in the gaps. In that sense, quantitative research is great because it gives us a starting point where we are likely to see something happen so we can start to watch it happen in the qualitative follow-up, but if we don't follow up with the qualitative, we miss the five percent who experience something else all together. On the other hand, if we start with qualitative because we really don't know what's happening but don't move to the quantative phase, we might not learn how unique the qualitative population might have been - that they were the five percent, not the 95 percent — and might not know that we spent a lot on the five percent and left the 95 percent to fend for themselves. Now, sure, research methods cannot be all things to all questions all the time, I get that, but they can't be only certain things to only certain questions at certain times; we need to be creative in how we investigate the human condition to truly understand it for the whole range of individuals - not just the five percent investigated in the qualitative work or the 95 percent investigated in the quantitative work. What I propose (and I'm sure I'm not alone, I just don't know the literature), is whether we table one for the other, we always need to circle back for what we left behind. They may be a statistical minority, but their experiences are not less valid and others may paint a beautiful picture, but miss the relevant and important experiences of the masses.

I'm sure this sounds like a silly rant of a graduate student, but if I think about my future workwhich I've been doing a lot of lately - I want to join the quest to unite quantitative and qualitative methods. And I don't just mean in a single study — although mixed methods is a great tool-I mean in the larger picture of various research lines and fields. We can do better and we should.

August 5, 2015

\section{FINGERS CROSSED I GET FUNDING FOR THIS RESEARCH}

Well, I just submitted a grant to the NCAA to fund my dissertation research. If I get it, I'll be 
able to fund undergraduate research assistants and buy technology to make transcription and analysis easier. One other thing I think was really useful about this process is that not only do I understand the grant submission process much better, it has really helped me conceptualize my study better. I actually feel like I can talk about it to people who don't know the area and make some sense. The bummer is that I'll have to wait until November to find out if I get it and I'm not sure if any undergraduates will want to help me out for free... guess we'll see...

September 11, 2015

\section{PARTICIPANT RECRUITMENT BEGINS}

Well, I sent out my first email to recruit participants yesterday. I had my fingers crossed I'd get one or two responses, but I already received six! Unfortunately, three of the responses were participants declining, but at least I heard from some people. There's no way recruiting participants can be this easy. I'm sure something will go wrong, but I'm feeling pretty good to know I have met my minimum in one day. I might actually graduate on time!

September 22, 2015

\section{SURPRISE, SURPRISE, I SPOKE TOO SOON}

So, recruiting participants isn't that easy. After several emails to contact the participants who agreed to participate in my study, I nailed one down for an interview time, then had to cancel on him because I was rushed into emergency surgery. This feels like a great start to my dissertation : I suppose I'll be sending out a second plea for participants later today.

September 25, 2015

\section{INTERVIEW \#1 DOWN... 5 MORE TO GO}

I just finished my first interview. It was a pretty fun experience. It was so nice to have a participant so open and willing to share his life with me. It was also really interesting to see how his mind operated and try to follow along. He had so many stories to share and while some of them were on topic, many were not, which made the whole situation interesting. Regardless, it really got me thinking about a lot of things. For one, even though I'm asking for very personal information, it is nice to see how open participants can be. I do get the feeling that he is hiding something because he seems to ramble on until he starts certain stories, then he gets really tightlipped and pauses to consider what he tells me, so I hope that comes out in the second interview. I will say, though, that it feels like what he's hiding may be more underconforming behavior than 
overconforming, so I'll be interested to see if he is more or less open during our next interview.

Also, he's brought up a lot of information about things unrelated to my study (e.g., demographic information that has shaped his wrestling experience). It makes me think a lot about future work and the intersection of various variables with overconforming behavior. Are we more likely to see certain kinds of overconforming behaviors from people with certain racial profiles or religious upbringings? Is the theory of overconformity that I'm investigating really showing us the whole picture, or is there a world of confounding variables out there that we have yet to discover. I'm afraid that if I use him as a case study, there would be no way to hide his identity, so I might need to work on seeing if I can get other participants from other schools who share similar traits, then do more of a case cohort study to protect his privacy and confidentiality. Clearly, this is not the way to go at this point - I just need to finish my dissertation — but it does bring some insights I wasn't expecting and I'd like to explore going forward.

October 6, 2015

\section{I'M GETTING DESPERATE!}

Despite my great start on day 1 of participant recruitment, I've only had one interview with three participants and am having trouble getting them to show for our agreed upon times. Not to mention, two of my participants are the same year, with quite similar backgrounds, so that's not good for maximum variation. I guess I'll be sending out a final plea to wrestlers not in that year to see if I can get anyone else to participate.

\section{SPEAKING OF MAXIMUM VARIATION...}

I wonder how I'll try and achieve maximum variation. Obviously, I'll look at year, weight class, and geographic region since I can get that information from the roster, but I probably want to get other information, as well. Based on the interviews I've done so far, I've heard about family athletic history, wins/losses, when wrestling started, and other sports, so I guess I could use those as variables to determine maximum variation, assuming I still get that information from my other participants. Only time will tell... and I'll ask Malayna!

\section{October 23, 2015}

\section{BEING DESPERATE WORKED—BUT PRESENTED NEW CHALLENGES}

So, my final sample is six participants. They represent four different years and six different weight classes, which is nice. I've been going back and forth about how to whittle them down to the three I'll analyze for this dissertation. I think I'm on the right track, given that I got a lot of information, so I should be able to plug each of them into a table and choose them objectively. 
Another complication is that two of the six did all of their data collection in one day, and that I couldn't stay to the one week between interviews I had originally proposed. Even though I had good reasons for conducting the interviews one week apart (i.e., so they had time to reflect and could clarify, if desired), research isn't always clean, so I guess I'll just have to go with it the way it is. I'm thinking that, at least in terms of reflection and clarification, member checking will allow me to do that, so that makes life easier. I suppose that if this is the worst that happened to me throughout this process, I'm probably in pretty good shape.

\section{REALLY HOPING I GET THE NCAA GRANT!}

Now that I have the data, I need to transcribe it all and I feel like I may not have completely understood how much information I was going to get from my interviews. I have one more participant's full interview to do, and another one's second half, so I'm hoping I get the type of good information I've received from other participants, but I definitely need help transcribing it or I'll never graduate in time!

December 1, 2015

\section{SO THAT'S A "NO" ON THE GRANT}

So I didn't get the NCAA grant, which sucks (like, really, really sucks!), but luckily I've had a lot of students helping me transcribe my data. I also found out that I didn't have to be in the same room as them when they transcribed because the data was de-identified, so that's freed up a lot of time. I know Watson thinks I'm crazy for transcribing all six participants' data, and he has a point that I can't analyze the data I'm not using for my dissertation the same way, but I still haven't figured out which three participants to use yet and I have more offers for help than I know what to do with, so I might as well take advantage of it. I suppose this means I'll really have to buckle down and figure out what variables I'll use for maximum variation so that I can choose my final sample!

December 13, 2015

\section{TRANSCRIPTION COMPLETE}

All data is transcribed, now I have to start analyzing it. I know I should do it over break, but the truth is that I'm tired (pregnancy is a lot hard with a 2 year old at home!) and need some time away from my dissertation to come back with fresh eyes. Plus, I have two students who want to code and help me write up, and they won't get started until January, so I might as well do it with them. 
January 15, 2016

\section{CODING OFFICIALLY BEGINS}

Today I begin coding. I've cleaned all of the transcripts and printed them out. I've also met with one of my undergraduate research assistants to coach her through the process and we did a few pages of a manuscript we aren't using to get her used to it. She seems pretty eager, so I'm excited to see how this goes-I've never had an undergraduate research assistant code qualitative data, so this is new for me, too.

I'm using the same coding scheme I used for my pilot interview. I assume the process will be the same and can't wait to see what the data says!

January 18, 2016

\section{HOPE I'M NOT A BAD RESEARCHER}

As I'm coding, I find myself making notes about reflections I'm having to either (a) myself as a researcher and things I wish I would have done differently or (b) things I want to make sure I don't forget to think about during later analysis/write up. I looked back at what I did with my pilot data, and I reflected on each of those things in analytic memos. How on earth am I supposed to do that with this much data?! It would be so much smarter to write all of that stuff down in these analytic memos, but it just feels too cumbersome. How do I find the middle ground because I don't think I can do line by line reflections with six interviews?!

January 25, 2016

\section{DOES CODING EVER END?!}

Well, we've been coding for weeks! My RA and I are on the same page and its really nice to see her challenge me. The interesting thing has been to compare how she interprets the theory and how I do. I mean, theory did form the basis of the provisional codes, so it is interesting to hear how she interprets distinction (i.e., really just as wins and losses) versus how I do (i.e., as anything that gains one distinction from his peers). It has really forced me to look into my own biases and understand where I'm coming from. Not only do I have personal experience with the concepts we are discussing, but I've been all through the literature, which means my understanding of it is different than hers, considering this is all new to her.

Interestingly, she is a college athlete, so we have quite insightful talks about how some of the issues we are talking about while coding look in her sport and her own experiences with it. I think its been a good exercise for both of us since we kind of check each others' biases and really 
analyze how we're constructing the data. That said, its funny that I ended up with a research assistant who has a very similar personality to me and who overanalyzes things the way I do. Sometimes I wonder if we'll ever get through this data to get an end product, but it's been refreshing to have the conversations I've had with her. I can't imagine doing this alone!

\section{ANOTHER IDEA, TOO LITTLE, TOO LATE :}

If I was better, I'd be tape-recording our meetings to go back and analyze later. The whole process of mentoring someone else through the research process has been fun, but I think, philosophically, it has been a great exercise in not only supervision, but also researcher positioning and awareness. I really think, had I thought about it earlier, that our interactions could be a unique research project in themselves — had I only thought about that earlier! Perhaps in another professional life...

February 18, 2016

\section{TODAY IS THE DAY: WE'RE MAKING THE WORD CLOUD!}

Morgan sent me her final codes earlier this week and I was able to put everything together in a master file. It's crazy to see all of our hard work coding — for over a month! — complete. I'll walk her through the process of making the word cloud and let her have a go at it. We'll see how it turns out.

\section{February 23, 2016}

\section{THE MUCH-ANTICIPATED CREATIVE NON-FICTION}

So I decided on my opening scene (the Wendy's scene) long ago. I knew it encompassed so many things that were talked about in the interviews (e.g., the sacrifice, the discipline, weightcutting, family influence, the physical nature of the sport, playing through pain, prioritizing wrestling), so I was excited to start my creative non-fiction a few days ago. When I crafted that scene, I was so into it. I really felt the words just pouring out of my fingers (which was pretty easy because I also had a transcript that told me where to go). But now I find myself in an interesting situation. When I first tried this with my pilot data, I just wrote about what was discussed. Now that I'm synthesizing the data, though, I have to create a character. This is supposed to be a combination of a life story and a semi-structured interview, created from three different individuals. I can't do that unless I have a character. I hadn't thought about how difficult that would be. It is easy enough to choose the stories that represent what I studied, the ones that embody the codes that were prevalent in each interview. But its not easy to write them as a cohesive story. I find myself marking different events to keep myself on track, but that also breaks it up so I'm not sure it's altogether cohesive. I'm on my first draft today, but I'm thinking this may take me a few more drafts than I expected to be true to a creative non-fiction! 
February 29, 2016

\section{CREATIVE NON-FICTION COMPLETE}

Well, it's not quite what I expected when I set out on this journey, but writing this creative nonfiction was quite the exercise. Not only did writing, itself, help to further interpret the results, but it's helping me think about what's really important with my research. I will say, though, that I intended this story to read as one coherent story. Instead, it's looking like the traditional themedriven qualitative work I'm used to seeing. I'm just torn about how to change it. On the one hand, I feel like it needs to be chronological, and follow the narrative structure my participants provided, staying true to the methods I used and the data I received. On the other hand, I'm not sure how that method would be received in the academic community. I guess I'll keep tweaking until I figure it out.

March 9, 2016

\section{NEW CREATIVE NON-FICTION COMPLETE}

So after speaking with my research assistant, Watson, and Malayna, I decided to create a second creative nonfiction that is chronological in nature. I still have my reservations about how this, as a product, would be received by the academic field, but it feels much more harmonious than my first attempt. I think I was still trying to force my first attempt into what I've seen in the past, which made me lose the story. This way, I really feel like the participants' voices are heard. I really wanted to do this type of results presentation to appeal to the reader emotionally, to help him understand the struggles on a deeper level, and prompt him to act, and I think writing this way will help me to achieve that. I'm looking forward to getting feedback from my committee about what they thing about it!

March 11, 2016

\section{ASK AND YOU SHALL RECEIVE}

I wanted feedback about my creative nonfiction, and I got it! Although it seems like my story is a hit with Watson and Malayna, there is still concern that I'm not doing enough interpretation. I'm finding it hard to respond to that feedback (although it is entirely valid) because I feel like I don't want my interpretation to be the end-all-be-all on this subject. For one thing, despite my attempts to minimize bias, the reality is that I can't control it; I can only seek to be aware of it and be open with my reader about it so that he/she may choose to evaluate my interpretations in light of that bias. And, really, the nice thing about creative nonfiction is that it invites participants to form their own interpretations. I had hoped that by staying true to their voices and mannerisms in text, the reader could evaluate for himself/herself what he/she thought and felt about the experiences. Because this area is so underresearched, one of my goals with this project is to get others to engage with it in a way that prompts them to take action, either by conducting future research or 
improving existing practice efforts. I am worried that if I do all of the work and present my results as if I know what is best, I know exactly how my participants evolved throughout their sport careers to eventually engage in overconformity, that I am a fraud. I only have the information they gave me to work with. All I can do is interpret that, in light of the existing research I know, and present another piece to the puzzle. Perhaps pregnancy is making me take all of this too far and is putting me in yet another existential crisis, but I'm finding myself very confused right now. Do I do what I'm sure the academic establishment would prefer, because that is what has always been done and so what should continue to be done, or should I challenge the system to engage the research in a different way, hopefully to get a different result?

March 15, 2016

\section{A DIFFERENT TYPE OF PRODUCT FROM MY RESEARCH-I CAN'T WAIT TO SEE HOW THIS GOES OVER!}

It has been feeling, lately, like I didn't do enough on the front end to help shape my committee members' expectations about this whole creative nonfiction thing. I completely understand the concern that by presenting just the creative nonfiction as a finding, that the reader feels compelled to work too hard to interpret the data. I think Watson said it best when he said it was interpretation twice removed. In an effort to combat that feeling, without interpreting for my reader (which is one of the advantages of using this technique, Smith, McGannon, \& Williams, 2015; Smith \& Sparkes, 2014, but requires a paradigm shift on the reader's part), I've now decided to present my word cloud and creative nonfiction up front, but then also do a brief interpretation after the nonfiction to help the reader connect the dots between the data and the existing literature. I knew at the outset of this project, I was going to have difficulty balancing the theory/data influence, but balancing both deductive and inductive aims in one manuscript has been a real struggle. One thing I've decided to do, both to address the interpretation issue, and to better balance the deductive and inductive aims of the study, is to create a figure that represents my conceptualization of the process of identity development and engagement in overconformity. In my mind, this figure is a beautiful marriage of existing literature and my contribution to it. If I thought I was excited about getting feedback on my creative nonfiction, I am ecstatic about the idea of getting feedback with this diagram!

March 22, 2016

\section{THE FIGURE IS A SUCCESS(ISH) BUT NEEDS SOME TWEAKING}

So I still don't know how to balance the story with interpretation, and I feel like the discussion is redundant to the "interpretation" section in the results, but I'm putting that quandary on hold. After speaking with Malayna this morning, it seems my figure was a good idea. She, however, had a better idea to present the figure twice. In the first, I just depict the literature base surrounding overconformity to the sport ethic. Then, in the second, I adjust the figure to reflect what I contributed to the current base. I think this is a great idea, I'm just freaking out because 1) I don't have the space and 2) I don't have the time. I hope I can pull it off before my committee 
gets my document in a week!

April 13, 2016

\section{DISSERTATION DEFENDED!}

I defended my dissertation today! I got some great feedback about my document, which I think will really help me to get it published. In the coming weeks, I think my biggest issue is going to be how to decide whose comments to listen to (several times, my committee members disagreed about how I should handle certain situations, which makes things hard!). Regardless, the mentorship and support I have received throughout this process has been indescribable. I could not imagine a smoother transition into doctorhood! 
APPENDIX J 


\section{Extended References}

Allan, E.J. \& Madden, M. (2008). Hazing in view: College students at risk. Initial Findings from the National Study of Student Hazing. Retrieved from http://www.stophazing.org/wpcontent/uploads/2014/06/hazing_in_view_web1.pdf.

Allen, J.B. (2003). Social motivation in youth sport. Journal of Sport \& Exercise Psychology, 25, 551-567.

Andersen, T. \& Finucane, M. (2015). Aaron Hernandez found guilty of murder: Convicted in 2013 shooting death of Odin Lloyd, sentenced to life in prison. The Boston Globe. Retrieved from http://www.bostonglobe.com/metro/2015/04/15/hernandez-jurorsdeliberate-for-seventh-day/QUaFD2uTTYn65GsKTEY26O/story.html

Atkinson, M. (2011). Male athletes and the cult(ure) of thinness in sport. Deviant Behavior, 32, 224-256.

Baker, K. (1999). Heidi Guenther's short, tragic life - and death. Retrieved from http://www. sfgate.com/magazine/article/Heidi-Guenther-s-short-tragic-life-and-death-3490764.php.

Bamberg, M. (2012). Narrative practice in identity navigation. In J.A. Holstein and J.F. Gubrium (Eds.) Varieties of Narrative Analysis. Los Angeles, CA: SAGE Publications, Inc.

Ben-Yehuda, N. (1990). Positive and negative deviance: More fuel for a controversy. Deviant Behavior, 11, 221-243.

Bracken, N.M. (2012). Substance use: National study of substance use trends among NCAA college student-athletes. Indianapolis, IN: The National Collegiate Athletic Association.

Brewer, B.W., \& Cornelius, A.E. (2001). Norms and factorial invariance of the Athletic Identity Measurement Scale. Academic Athletic Journal, 16, 103-113.

Buckner, C. (2014). As 'Malice at the Palace" brawl turns 10, impact lasts. USA Today. Retrieved from http://www.usatoday.com/story/sports/nba/pacers/2014/11/16/pistons- 
malice-palace-brawl-commissioner-artest-adam-silver/19138239/

Carless, D. \& Douglas, K. (2013a). Living, resisting, and playing the part of athlete: Narrative tensions in elite sport. Psychology of Sport and Exercise, 14, 701-708.

Carless, D. \& Douglas, K. (2013b). "In the boat" but "selling myself short": Stories, narratives and identity development in elite sport. The Sport Psychologist, 27, 27-39.

Coakley, J. (2009). Deviance in sports: Is it out of control? In J. Coakley (Ed.) Sports in Society: Issues and Controversies, pp. 152-193. New York, NY: McGraw-Hill.

Coakley, J. (2015). Drug use and deviant overconformity: A sociological approach. In J. Hoberman, I. Waddington, \& Moller, V. (Eds.) The Routledge Companion to Sport and Drugs, pp.379-392. London: Routledge.

Cottler, L.B., Abdallah, A.B., Cummings, S.M., Barr, J., Banks, R., \& Forchheimer, R. (2011). Injury, pain, and prescription opioid use among former National Football League (NFL) players. Drug and Alcohol Dependence, 116, 188-194.

Creswell, J.W. (2007). Qualitative Inquiry and Research Design: Choosing Among Five Approaches ( $\left.2^{\text {nd }} e d\right)$. Thousand Oaks, CA: SAGE Publications, Inc.

Creswell, J.W. (2009). Research Design: Qualitative, Quantitative, and Mixed Methods Approaches $\left(3^{\text {rd }}\right.$ ed). Thousand Oaks, CA: SAGE Publications, Inc.

Crotty, M.J. (1998). The Foundations of Social Research: Meaning and Perspective in the Research Process. Thousand Oaks, CA: SAGE Publications, Inc.

Curry, T.J. (1993). A little pain never hurt anyone: Athletic career socialization and the normalization of sports injury. Symbolic Interaction, 16(3), 273-290.

Dodge, D.L. (1985). The over-negativised conceptualization of deviance: A programmatic exploration. Deviant Behavior, 6, 17-37. 
Donnelly, P. (2004). Sport and risk culture. In K. Young (Ed.) Sporting Bodies, Damaged Selves: Sociological Studies of Sports-Related Injury, pp. 29-58. San Diego, CA: ELSEVIER, Inc.

Donnelly, P. \& Young, K. (1988). The construction and confirmation of identity in sport subcultures. Sociology of Sport Journal, 5, 223-240.

Duda, J.L. \& Nicholls, J. (1992). Dimensions of achievement motivation in schoolwork and sport. Journal of Educational Psychology, 84, 290-299.

Dunn, M., Thomas, J.O., Swift, W., \& Burns, L. (2011). Elite athletes' estimates of the prevalence of illicit drug use: Evidence for the false consensus effect. Drug and Alcohol Review, 31, 27-32.

Esin, C., Fathi, M., \& Squire, C. (2013). Narrative analysis: The constructionist approach. In U. Flick (Ed.), The SAGE Handbook of Qualitative Data Analysis. Thousand Oaks, CA: SAGE Publications, Inc.

Feinberg, J. (2013). Wordle ${ }^{\mathrm{TM}}$. Retrieved from www.wordle.net on March 13, 2015.

Fenton, L.T. \& Pitter, R. (2010). Keeping the body in play: Pain, injury, and socialization in male rugby. Research Quarterly for Exercise and Sport, 81(2), 212-223.

Frank, A.W. (2010). Letting Stories Breathe: A Socio-Narratology. Chicago, IL: The University of Chicago Press.

Gould, I. (2013). Saban's 2013 BCS national Championship final press conference. Retrieved from http://www.al.com/alabamafootball/index.ssf/2013/01/sabans_2013_bcs_national_ champ.html.

Ganim, S. \& Sayers, D. (2014). UNC report finds 18 years of academic fraud to keep athletes playing. CNN Investigations. Retrieved from http://www.cnn.com/2014/10/22/us/unc- 
report-academic-fraud/

Gibbs, A.E., Pickerman, J., \& Sekiya, J.K. (2009). Weight management in amateur wrestling. Sports Health, 1(3), 227-230.

Greenleaf, C., Petrie, T.A., Carter, J., \& Reel, J.J. (2009). Female collegiate athletes: Prevalence of eating disorders and disordered eating behaviors. Journal of American College Health, $57(5), 489-496$.

Hale, B.D. \& Waalkes, D. (1994). Athletic identity, gender, self esteem, academic importance, and drug use: A further validation of the AIMS. [Abstract] Journal of Sport \& Exercise Psychology, 16(S), S62.

Heckert \& Heckert (2002). A new typology of deviance: integrating normative and reactivist definitions of deviance. Deviant Behavior: An Interdisciplinary Journal, 23, 449-479.

Hewitt, B. (1997). Last dance. Retrieved from http://www.people.com/people/archive/article/ 0,,20122756,00.html.

Heil, J. (2012). Pain on the run: Injury, pain, and performance in a distance runner. The Sport Psychologist, 26, 540-550.

Hoover, N.C. (1999). National survey: Initiation rites and athletics for NCAA sports teams. Available from http://www.alfred.edu/sports_hazing/docs/hazing.pdf.

Hughes, R. \& Coakley, J. (1991). Positive deviance among athletes: The implications of overconformity to the sport ethic. Sociology of Sport Journal, 8, 307-325.

Johns, D. (1993). Nutritional need or athletic overconformity: Ethical implications for the sport psychologist. The Sport Psychologist, 7, 191-203.

Johns, D. (1998). Fasting and feasting: Paradoxes of the sport ethic. Sociology of Sport Journal, 15, 41-63. 
King, S., Carey, R.S., Jinnah, N., Milington, R., Phillipson, A., Prouse, C., \& Ventresca, M. (2014). When is a drug not a drug? Troubling silences and unsettling painkillers in the National Football League. Sociology of Sport Journal, 31, 249-266.

Kowalski, C. \& Waldron, J. (2010). Looking the other way: Athletes' perceptions of coaches' responses to hazing. International Journal of Sports Science \& Coaching, 5(1), 2010.

Kraus, W. (2007). The narrative negotiation of identity and belonging. Narrative Inquiry, 16(1), 103-111.

Lieblich, A., Tuval-Mashiach, R., \& Zilber, T. (1998). Narrative Research: Reading, Analysis, and Interpretation. Thousand Oaks, CA: SAGE Publications, Inc.

Loenneke, J.P., Wilson, J.M., Barnes, J.T., \& Pujol, T.J. (2011). Validity of the current NCAA minimum weight protocol: A brief review. Annals of Nutrition and Metabolism, 58, 245249.

Marshall, C. \& Rossman, G.B. (2006). Designing Qualitative Research. Thousand Oaks, CA: SAGE Publications, Inc.

McAdams, D.P. (1998). The role of defense in the life story. Journal of Personality, 66(6), 11251146.

McAdams, D.P. (2001). The psychology of life stories. Review of General Psychology, 5(2), $100-122$.

McAdams, D.P. (2012). Exploring psychological themes through life-narrative accounts. In J.A. Holstein and J.F. Gubrium (Eds.) Varieties of Narrative Analysis. Los Angeles, CA: SAGE Publications, Inc.

McAdams, D.P. \& Guo, J. (2014). How shall I live? Constructing a life story in the college years. New Directions for Higher Education, 166, 15-23. 
McNaught, C. \& Lam, P. (2010). Using Wordle as a supplementary research tool. The Qualitative Report, 15(3), 630-643.

Morrow, S.L. (2005). Quality and trustworthiness in qualitative research in counseling psychology. Journal of Counseling Psychology, 52(2), 250-260.

Morrow, S.L. (2007). Qualitative research in counseling psychology: Conceptual foundations. The Counseling Psychologist, 35(2), 209-235.

Nixon, H.L. (1992). A social network analysis of influences on athletes to play with pain and injuries. Journal of Sport and Social Issues, 16, 127-135.

Nixon, H.L. (1993). Accepting the risks of pain and injury in sport: Mediated cultural influences on playing hurt. Sociology of Sport Journal, 10, 183-196.

Opplinger, R.A., Nelson Steen, S.A., \& Scott, J.R. (2003). Weight loss practices of college wrestlers. International Journal of Sport Nutrition and Exercise Metabolism, 13, 29-46.

Peacock, J.L. \& Holland, D.C. (1993). The narrated self: Life stories in process. Ethos, 21(4), 367-383.

Petrie, T.A., Greenleaf, C., Reel, J. \& Carter, J. (2008). Prevalence of eating disorders and disordered eating behaviors among male collegiate athletes. Psychology of Men \& Masculinity, 9(4), 267-277.

Pettersson, S., Ekstrom, M.P., \& Berg, C.M. (2013). Practices of weight regulation among elite athletes in combat sports: A matter of mental advantage? Journal of Athletic Training, 48(1), 99-108.

Ponterotto, J.G. (2005) Qualitative research in counseling psychology: A primer on research paradigms and philosophy of science. Journal of Counseling Psychology, 52(2) 126-136.

Rexroat, M. (2014). NCAA national study of substance use habits of college student athletes: 
Final report. Retrieved from http://www.ncaa.org/sites/default/files/Substance\%20Use\% 20Final\%20Report_FINAL.pdf.

Reynolds, M.J. (2000). A theoretical exploration of the relationship between the expectations of sports coaches and the physical and emotional health of athletes. Journal of Science \& Medicine in Sport, 3(2 S1), 51-57.

Sagarin, E. (1985). Positive deviance: An oxymoron. Deviant Behavior, 6, 169-181.

Schnell, A., Mayer, J., Diehl, K., Zipfel, S. Thiel, A. (2014). Giving everything for athletic success! - Sports-specific risk acceptance of elite adolescent athletes. Psychology of Sport and Exercise, 15, 165-172.

Smith, B. (2010). Narrative inquiry: Ongoing conversations and questions for sport and exercise psychology research. International Review of Sport and Exercise Psychology, 3(1), 87107.

Smith, B. \& Sparkes, A.C. (2009a). Narrative inquiry in sport and exercise psychology: What can it mean and why might we do it? Psychology of Sport and Exercise, 10, 1-11.

Smith, B. \& Sparkes, A.C. (2009b). Narrative analysis and sport and exercise psychology: Understanding lives in diverse ways. Psychology of Sport and Exercise, 10, 279-288.

Sparkes, A. (2005). Narrative analysis: Exploring the whats and the hows of personal stories. In M. Holloway (Ed.), Qualitative Research in Health Care (pp. 91-209). Milton Keynes: Open University Press.

Sparkes, A.C. \& Smith, B. (2012). Narrative analysis as embodied engagement with the lives of others. In J.A. Holstein and Gubrium, J.F. (Eds.), Varieties in Narrative Analysis. Los Angeles, CA: SAGE Publications, Inc.

Sparkes, A.C. \& Smith, B. (2014). Qualitative Research Methods in Sport, Exercise and Health: 
From Process to Product. New York, NY: Routledge.

Waldron, J.J. \& Krane, V. (2005). Whatever it takes: Health compromising behaviors in female athletes. QUEST, 57, 315-329.

Waldron, J.J. \& Kowalski, C.L. (2009). Crossing the line: Rites of passage, team aspects, and ambiguity of hazing. Research Quarterly for Exercise and Sport, 80(2), 291-302.

Waldron, J.J., Lynn, Q., \& Krane, V. (2011). Duct tape, icy hot \& paddles: Narratives of initiation onto US male sport teams. Sport, Education and Society, 16(1), 111-125.

Weinberg, R. (n.d.) Kerri Strug fights off the pain, helps U.S. win gold. Retrieved from http://espn.go.com/espn/espn25/story?page=moments/51.

Vajda, J. (2007). Two survivor cases: Therapeutic effect as a side product of the biographical narrative interview. Journal of Social Work Practice, 21(1), p. 89-102.

Vetter, R.E. \& Symonds, M.L. (2010). Correlations between injury, training intensity, and physical and mental exhaustion among college athletes. Journal of Strength \& Conditioning Research, 24(3), 587-596.

Yeh, C.J. \& Inman, A.G. (2007). Qualitative data analysis and interpretation in counseling psychology: Strategies for best practices. The Counseling Psychologist, 35(3), 369-403.

Young, K. (1993). Violence, risk, and liability in male sports culture. Sociology of Sport Journal, 10, 373-396.

Young, K., McTeer, W. \& White, P. (1994). Body talk: Male athletes reflect on sport, injury, and pain. Sociology of Sport Journal, 11, 175-194. 